\title{
Utilizing Native Directing Groups: Mechanistic Understanding of a Direct Arylation Leads to Formation of Tetracyclic Heterocycles via Tandem Intermolecular, Intramolecular C-H Activation
}

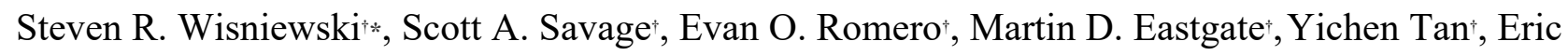
M. Simmons ${ }^{\dagger}$ R. Erik Plata ${ }^{\ddagger}$ John R. Sowa, Jr., Donna G. Blackmond

'Chemical and Synthetic Development, Bristol-Myers Squibb, One Squibb Drive, New Brunswick, New Jersey 08903, United States

${ }^{\ddagger}$ Department of Chemistry, The Scripps Research Institute, 10550 North Torrey Pines Road, La Jolla, California 92037, United States

\author{
steven.wisniewski@bms.com \\ Supporting Information
}

Table of Contents:

Optimization of the C-H Activation...................................S2

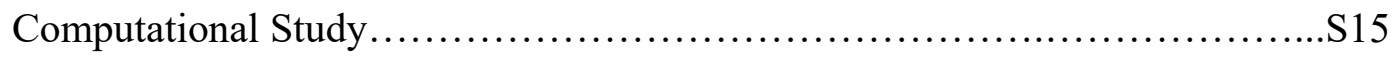

Crystal Structure of Palladacycle ...................................S23

Competency of the Palladcycle in the C-H Activation.......................S25

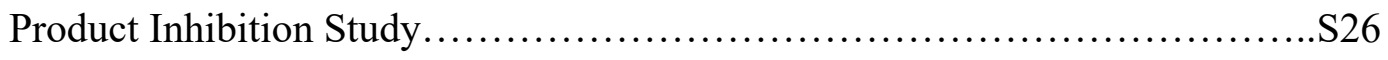

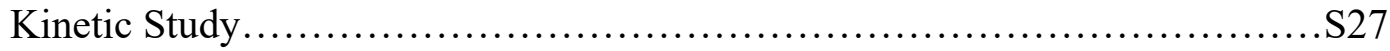

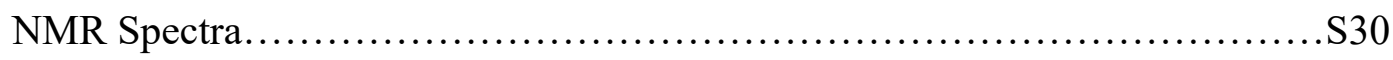


Optimization of the C-H Activation:<smiles>CC(C)(C)NS(=O)(=O)c1cncc(-c2nc(NCc3ccccn3)c3ccccc3n2)c1</smiles><smiles>CC(C)(C)NS(=O)(=O)c1cncc(-c2nc(NCc3ccccn3)c3c(-c4ccccc4)cccc3n2)c1</smiles>

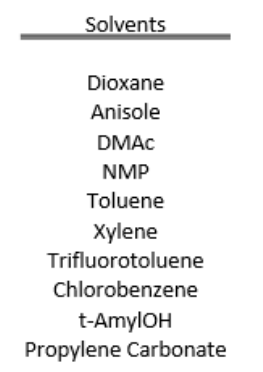

$$
\begin{gathered}
\text { KHCO3 } \\
\mathrm{Cs} 2 \mathrm{CO} 3 \\
\mathrm{~K} 2 \mathrm{CO} 3 \\
\mathrm{NaHCO} 3 \\
\mathrm{Na} 2 \mathrm{CO} 3 \\
\mathrm{~K} 3 \mathrm{PO} 4 \\
\mathrm{~K} 2 \mathrm{HPO} 4 \\
\mathrm{KH} 2 \mathrm{PO} 4 \\
\mathrm{CsF} \\
\mathrm{KF} \\
\mathrm{NaOMe} \\
\text { KOtBu } \\
\text { KOPiv } \\
\text { KOAC } \\
\text { TMAOAC } \\
\text { NEt3 } \\
\text { 2,6-lutidene } \\
\text { Cy2NMe } \\
\text { DIPEA } \\
\text { DBU } \\
\text { DABCO }
\end{gathered}
$$

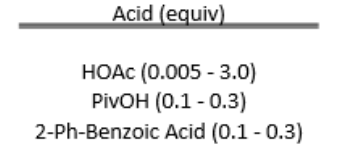

2-Ph-Benzoic Acid $(0.1-0.3)$
Dibenzyl phosphate $(0.1-0.3)$
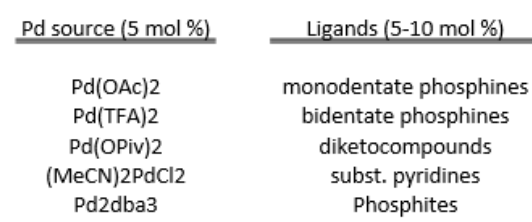

lodobenzene Phenyl Triflate Bromobenzene

General Procedure for High Throughput Screening. In the glovebox, pre-dosed ligand vials are added to the reaction plate (if applicable). A solution of catalyst ( $25 \mu \mathrm{L}$ of a $0.02 \mathrm{M}$ solution of $\mathrm{Pd}(\mathrm{OAc})_{2}$ in THF $)$ is then added, and the plate is aged at $\mathrm{rt}$ for $20 \mathrm{~min}$. A solution of substrate ( $50 \mu \mathrm{L}$ of a $0.2 \mathrm{M}$ solution in tetrahydrofuran) is then added. Non-volatile additives or cocatalysts are then added as solutions in THF. The solvent is removed via the genevac. Stir bars are added to the plate followed by solid dispensing of the base. Solvent and iodobenzene are then added. Note: if a volatile ligand or additive is used, it is added a solution in the reaction solvent at this stage. The plate is sealed with a Teflon sheet and lid, removed from the glovebox and mixed at $95-120^{\circ} \mathrm{C}$ overnight. After $16 \mathrm{~h}$, the vials are diluted with 3:1 NMP/MeCN $(500 \mu \mathrm{L})$. An aliquot $(40 \mu \mathrm{L})$ is further diluted into $\mathrm{MeCN}(1 \mathrm{~mL})$ and subjected to UPLC analysis. 


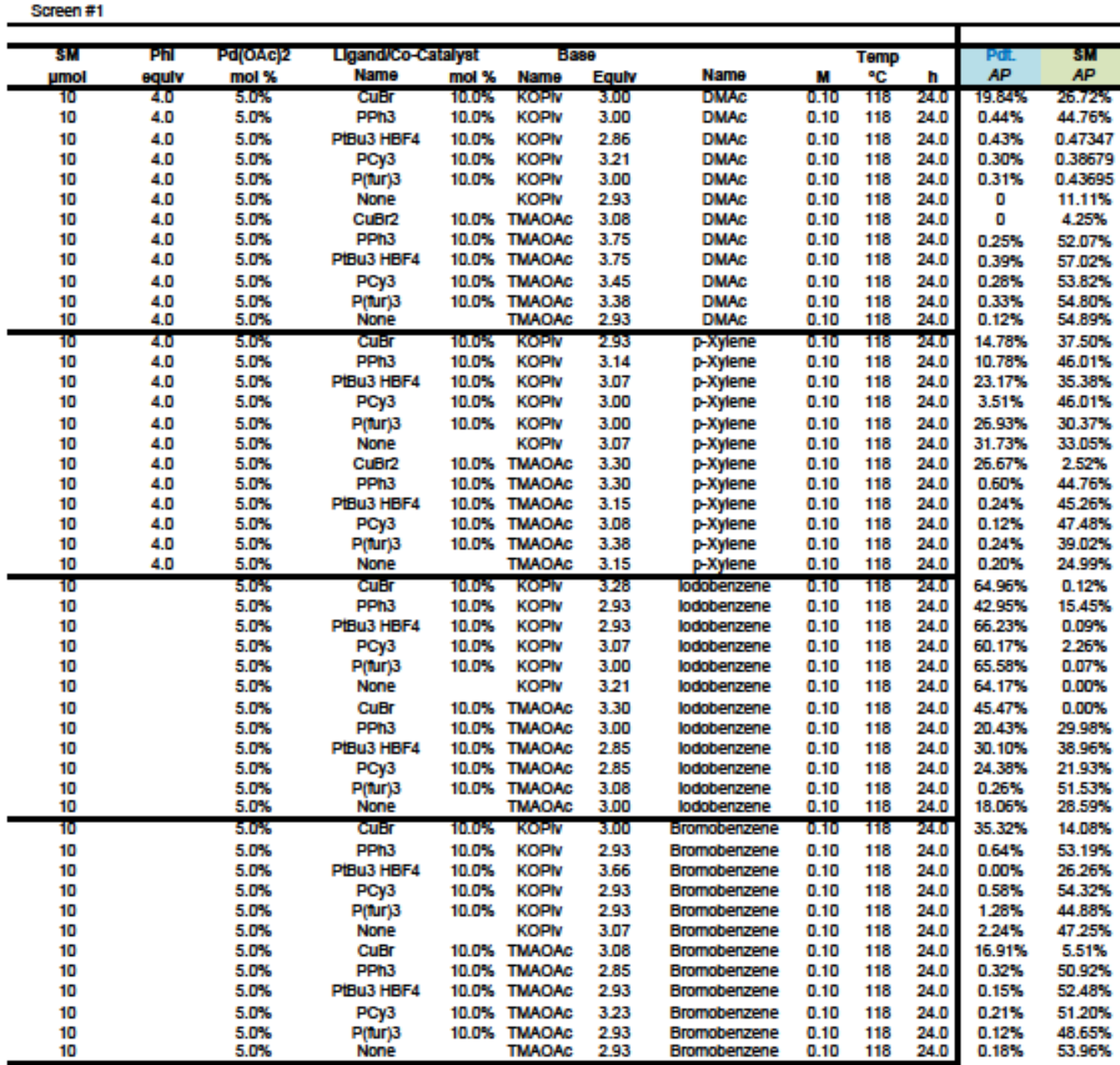


seen \#1, cont

\begin{tabular}{|c|c|c|c|c|c|c|c|c|c|c|c|c|}
\hline \multirow{2}{*}{$\begin{array}{l}\text { sM } \\
\text { umol }\end{array}$} & \multirow{2}{*}{$\begin{array}{c}\text { Phi } \\
\text { Name }\end{array}$} & \multirow{2}{*}{$\begin{array}{c}\text { Pd(OAC) } 2 \\
\text { umol }\end{array}$} & \multirow{2}{*}{\multicolumn{2}{|c|}{$\begin{array}{l}\text { Ugand/co-catalyzt } \\
\text { Name mol \% }\end{array}$}} & \multicolumn{2}{|c|}{ Bख80 } & \multirow[b]{2}{*}{ Name } & \multirow{2}{*}{\multicolumn{3}{|c|}{ Temp }} & \multirow{2}{*}{$\begin{array}{l}\text { Pait } \\
\text { AP }\end{array}$} & \multirow{2}{*}{ SMP } \\
\hline & & & & & Name & Equlv & & M & ${ }^{\circ} \mathrm{C}$ & & & \\
\hline 10 & 4.0 & $5.0 \%$ & $\begin{array}{l}\text { Cuer } \\
\text { Pph } 3\end{array}$ & $10.0 \%$ & KOAAC & 2.85 & DIMAC & 0.10 & 118 & 24.0 & $5.88 \%$ & $38.09 \%$ \\
\hline 10 & 4.0 & $5.0 \%$ & PPh3 & $10.0 \%$ & KOAC & 3.16 & DMAC & 0.10 & 118 & 24.0 & $1.08 \%$ & $19.91 \%$ \\
\hline 10 & 4.0 & $5.0 \%$ & PtBu3 HBF/ & $10.0 \%$ & KOAC & 3.36 & DMAC & 0.10 & 118 & 24.0 & $0.83 \%$ & $57.00 \%$ \\
\hline 10 & 4.0 & $5.0 \%$ & PCy3 & $10.0 \%$ & KOAC & 3.06 & DMAC & 0.10 & 118 & 24.0 & $0.49 \%$ & $59.91 \%$ \\
\hline 10 & 4.0 & $5.0 \%$ & $P($ tur $) 3$ & $10.0 \%$ & KOAC & 3.16 & DMAC & 0.10 & 118 & 24.0 & $0.15 \%$ & $53.32 \%$ \\
\hline 10 & 4.0 & $5.0 \%$ & None & & KOAC & 3.06 & DMAC & 0.10 & 118 & 24.0 & $0.34 \%$ & $58.18 \%$ \\
\hline 10 & 4.0 & $5.0 \%$ & CuBr & $10.0 \%$ & $\operatorname{cs} 2 \mathrm{CO} 3$ & 3.07 & DMAC & 0.10 & 118 & 24.0 & $20.73 \%$ & $14.92 \%$ \\
\hline 10 & 4.0 & $5.0 \%$ & PPh3 & $10.0 \%$ & $\mathrm{Cs} 2 \mathrm{CO} 3$ & 3.10 & DMAC & 0.10 & 118 & 24.0 & $2.47 \%$ & $23.94 \%$ \\
\hline 10 & 4.0 & $5.0 \%$ & PtBu3 HBF/ & $10.0 \%$ & $\operatorname{cs} 2 \mathrm{CO}_{3}$ & 3.10 & DMAC & 0.10 & 118 & 24.0 & $2.79 \%$ & $29.02 \%$ \\
\hline 10 & 4.0 & $5.0 \%$ & PCy3 & $10.0 \%$ & $\operatorname{cs} 2 \mathrm{CO}_{3}$ & 3.38 & DMAC & 0.10 & 118 & 24.0 & $1.71 \%$ & $18.95 \%$ \\
\hline $\begin{array}{l}10 \\
10\end{array}$ & $\begin{array}{l}4.0 \\
4.0\end{array}$ & $\begin{array}{l}5.0 \% \\
5.0 \%\end{array}$ & $\begin{array}{l}\text { P(fur) } 3 \\
\text { None }\end{array}$ & $10.0 \%$ & $\begin{array}{l}\mathrm{Cs} 2 \mathrm{CO} 3 \\
\mathrm{Cs} 2 \mathrm{CO} 3\end{array}$ & $\begin{array}{l}3.10 \\
3.22\end{array}$ & $\begin{array}{l}\text { DMAC } \\
\text { DMAC }\end{array}$ & $\begin{array}{l}0.10 \\
0.10\end{array}$ & $\begin{array}{l}118 \\
118\end{array}$ & $\begin{array}{l}24.0 \\
24.0\end{array}$ & $\begin{array}{l}1.46 \% \\
1.18 \%\end{array}$ & $\begin{array}{l}26.17 \% \\
17.36 \%\end{array}$ \\
\hline 10 & 4.0 & $5.0 \%$ & cuer & $70.0 \%$ & KOAC & 2.96 & p-xylene & 0.10 & 118 & 24.0 & $10.58 \%$ & $43.41 \%$ \\
\hline 10 & 4.0 & $5.0 \%$ & PPh3 & $10.0 \%$ & KOAC & 3.16 & p-xylene & 0.10 & 118 & 24.0 & $0.37 \%$ & $35.56 \%$ \\
\hline 10 & 4.0 & $5.0 \%$ & PtBu3 HBF/ & $10.0 \%$ & KOAC & 3.06 & p-xylene & 0.10 & 118 & 24.0 & $4.33 \%$ & $26.03 \%$ \\
\hline 10 & 4.0 & $5.0 \%$ & PCy3 & $10.0 \%$ & KOAC & 4.08 & p-xylene & 0.10 & 118 & 24.0 & $0.47 \%$ & $56.68 \%$ \\
\hline 10 & 4.0 & $5.0 \%$ & $P(\text { tur })^{3}$ & $10.0 \%$ & KOAC & 3.06 & p-Xylene & 0.10 & 118 & 24.0 & $1.16 \%$ & $47.47 \%$ \\
\hline 10 & 4.0 & $5.0 \%$ & None & & KOAC & 3.06 & p-xylene & 0.10 & 118 & 24.0 & $14.02 \%$ & $46.57 \%$ \\
\hline 10 & 4.0 & $5.0 \%$ & CuBr & $10.0 \%$ & $\operatorname{Cs} 2 \mathrm{CO}_{3}$ & 2.97 & p-xylene & 0.10 & 118 & 24.0 & $6.27 \%$ & $8.83 \%$ \\
\hline 10 & 4.0 & $5.0 \%$ & PPh3 & $10.0 \%$ & $\operatorname{cs} 2 \mathrm{CO}_{3}$ & 2.97 & p-xylene & 0.10 & 118 & 24.0 & $0.25 \%$ & $3.08 \%$ \\
\hline 10 & 4.0 & $5.0 \%$ & PtBu3 HBF/ & $10.0 \%$ & $\mathrm{Cs} 2 \mathrm{CO} 3$ & 3.35 & p-xylene & 0.10 & 118 & 24.0 & $0.72 \%$ & $20.73 \%$ \\
\hline 10 & 4.0 & $5.0 \%$ & PCy3 & $10.0 \%$ & $\operatorname{cs} 2 \mathrm{CO}_{3}$ & 3.16 & p-xylene & 0.10 & 118 & 24.0 & $0.15 \%$ & $42.08 \%$ \\
\hline 10 & 4.0 & $5.0 \%$ & $P(\text { tur })^{3}$ & $10.0 \%$ & $\operatorname{cs} 2 \mathrm{CO}_{3}$ & 2.85 & p-xylene & 0.10 & 118 & 24.0 & $0.52 \%$ & $45.12 \%$ \\
\hline 10 & 4.0 & $5.0 \%$ & None & & $\operatorname{cs} 2 \mathrm{CO}_{3}$ & 3.50 & p-xylene & 0.10 & 118 & 24.0 & $0.33 \%$ & $28.40 \%$ \\
\hline 10 & & $5.0 \%$ & cuter & $10.0 \%$ & KOAC & 2.96 & odobenzen: & 0.70 & 118 & 24.0 & $57.55 \%$ & $3.23 \%$ \\
\hline 10 & & $5.0 \%$ & PPh3 & $10.0 \%$ & KOAC & 2.85 & odobenzen: & 0.10 & 118 & 24.0 & $6.54 \%$ & $41.65 \%$ \\
\hline 10 & & $5.0 \%$ & PtBu3 HBF/ & $10.0 \%$ & KOAC & 3.06 & odobenzen: & 0.10 & 118 & 24.0 & $28.01 \%$ & $49.95 \%$ \\
\hline 10 & & $5.0 \%$ & PCy3 & $10.0 \%$ & KOAC & 3.26 & odobenzen: & 0.10 & 118 & 24.0 & $17.68 \%$ & $54.14 \%$ \\
\hline 10 & & $5.0 \%$ & $P($ úr) 3 & $10.0 \%$ & KOAC & 3.26 & odobenzen: & 0.10 & 118 & 24.0 & $21.23 \%$ & $28.51 \%$ \\
\hline 10 & & $5.0 \%$ & None & & KOAC & 2.85 & odobenzen: & 0.10 & 118 & 24.0 & $76.90 \%$ & $0.76 \%$ \\
\hline 10 & & $5.0 \%$ & CuEr & $10.0 \%$ & $\operatorname{cs} 2 \mathrm{CO} 3$ & 3.41 & odobenzen: & 0.10 & 118 & 24.0 & $59.55 \%$ & $14.78 \%$ \\
\hline 10 & & $5.0 \%$ & PPh3 & $10.0 \%$ & $\operatorname{cs} 2 \mathrm{CO}_{3}$ & 3.22 & odobenzen: & 0.10 & 118 & 24.0 & $9.88 \%$ & $8.41 \%$ \\
\hline 10 & & $5.0 \%$ & PtBu3 HBF/ & $10.0 \%$ & $\operatorname{cs} 2 \mathrm{CO} 3$ & 2.97 & odobenzen: & 0.10 & 118 & 24.0 & $9.04 \%$ & $50.58 \%$ \\
\hline 10 & & $5.0 \%$ & PCy3 & $10.0 \%$ & $\mathrm{Cs} 2 \mathrm{CO} 3$ & 3.07 & odobenzen: & 0.10 & 118 & 24.0 & $5.02 \%$ & $56.15 \%$ \\
\hline $\begin{array}{l}10 \\
10\end{array}$ & & $\begin{array}{l}5.0 \% \\
5.0 \%\end{array}$ & $\begin{array}{l}\text { P(fur) } 3 \\
\text { None }\end{array}$ & $10.0 \%$ & $\begin{array}{l}\mathrm{Cs} 2 \mathrm{CO} 3 \\
\mathrm{Cs} 2 \mathrm{CO} 3\end{array}$ & $\begin{array}{l}3.10 \\
3.07\end{array}$ & $\begin{array}{l}\text { odobenzen: } \\
\text { odobenzen: }\end{array}$ & $\begin{array}{l}0.10 \\
0.10\end{array}$ & $\begin{array}{l}118 \\
118\end{array}$ & $\begin{array}{l}24.0 \\
24.0\end{array}$ & $\begin{array}{l}8.73 \% \\
19.10 \%\end{array}$ & $\begin{array}{l}43.99 \% \\
41.39 \%\end{array}$ \\
\hline $10^{\circ}$ & & $5.0 \%$ & cuer & $70.0 \%$ & KOAAC & 357 & romobenzer & 0.10 & 118 & 24.0 & $9.39 \%$ & $27.26 \%$ \\
\hline 10 & & $5.0 \%$ & PPh3 & $10.0 \%$ & KOAC & 2.96 & romobenzer & 0.10 & 118 & 24.0 & $0.38 \%$ & $45.23 \%$ \\
\hline 10 & & $5.0 \%$ & PtBu3 HBF/ & $10.0 \%$ & KOAC & 3.06 & romobenzer & 0.10 & 118 & 24.0 & $0.54 \%$ & $49.05 \%$ \\
\hline 10 & & $5.0 \%$ & PCy3 & $10.0 \%$ & KOAC & 2.96 & romobenzer & 0.10 & 118 & 24.0 & $0.44 \%$ & $48.70 \%$ \\
\hline 10 & & $5.0 \%$ & $P(\text { tur })^{3}$ & $10.0 \%$ & KOAC & 3.16 & romobenzer & 0.10 & 118 & 24.0 & $0.55 \%$ & $38.60 \%$ \\
\hline 10 & & $5.0 \%$ & None & & KOAC & 3.26 & romobenzer & 0.10 & 118 & 24.0 & $0.26 \%$ & $50.01 \%$ \\
\hline 10 & & $5.0 \%$ & Cuer & $10.0 \%$ & $\operatorname{cs} 2 \mathrm{CO}_{3}$ & 3.10 & romobenzer & 0.10 & 118 & 24.0 & $16.62 \%$ & $8.07 \%$ \\
\hline 10 & & $5.0 \%$ & PPh3 & $10.0 \%$ & $\operatorname{cs} 2 \mathrm{CO}_{3}$ & 3.10 & romobenzer & 0.10 & 118 & 24.0 & $0.00 \%$ & $30.94 \%$ \\
\hline 10 & & $5.0 \%$ & PtBu3 HBF/ & $10.0 \%$ & $\mathrm{Cs} 2 \mathrm{CO} 3$ & 3.16 & romobenzer & 0.10 & 118 & 24.0 & $0.36 \%$ & $32.01 \%$ \\
\hline 10 & & $5.0 \%$ & PCy3 & $10.0 \%$ & $\operatorname{cs} 2 \cos _{3}$ & 2.97 & romobenzer & 0.10 & 118 & 24.0 & $0.06 \%$ & $44.40 \%$ \\
\hline 10 & & $5.0 \%$ & $P$ (fur) 3 & $10.0 \%$ & $\mathrm{Cs}_{2} \mathrm{CO}_{3}$ & 3.00 & romobenzer & 0.10 & 118 & 24.0 & $0.19 \%$ & $39.41 \%$ \\
\hline 10 & & $5.0 \%$ & None & & $\operatorname{Cs} 2 \mathrm{CO}_{3}$ & 3.10 & romobenzer & 0.10 & 118 & 24.0 & $0.05 \%$ & $42.13 \%$ \\
\hline
\end{tabular}




\begin{tabular}{|c|c|c|c|c|c|c|c|c|c|c|c|c|}
\hline sin & & & & & & abdarive & & & Irmp & & Pait & sim \\
\hline mol & equilv & $6 \mathrm{~mol} \mathrm{H}$ & $6 \mathrm{~mol} \%$ & Name & Equlv & $10 \mathrm{~mol} / \mathrm{6}$ & Name & vol & ${ }^{\circ} \mathrm{C}$ & $\mathrm{h}$ & $A P$ & $A P$ \\
\hline 10 & 4.0 & Fo[OरC:2 & FiBDBHAEF-4 & ROOPN & 328 & cuer2 & Suzup-xyenelosobenzene & 74.73 & 705 & 24.0 & 55.5456 & $30.16 \%$ \\
\hline 10 & 4.0 & PdiOAci2 12 & PtBU 3 HEF 4 & KOPN & 3.14 & $\mathrm{CuCl2}$ & 5050 p-Xylene:lodobenzune & 14.73 & 105 & 24.0 & $49.31 \%$ & $25.80 \% 6$ \\
\hline 10 & 4.0 & PdiOAci2 & PtBU 3 HEF 4 & KOPN & 3.07 & Cul & 5050 p-Xylene:lodobenzene & 14.73 & 105 & 24.0 & $49.25 \%$ & 0.36226 \\
\hline 10 & 4.0 & PdiOAci2 & PtBu 3 HEF 4 & KOPN & 3.00 & cuca & 5050 p-Xylene:lodobenzene & 14.73 & 105 & 24.0 & $55.20 \%$ & 0.25413 \\
\hline 10 & 4.0 & PdiOACI2 & PLBU 3 HEF 4 & KOPN & 2.93 & ano & $50-50$ p-Xylene:lodobenzene & 14.73 & 105 & 24.0 & $4429 \%$ & 0.3637 \\
\hline 10 & 4.0 & PdiOAcis & PtBU 3 HBF 4 & KOPN & 3.00 & CulOAc)2 2 & $50-50$ p-Xyleneilodoberzene & 14.73 & 105 & 24.0 & 0 & $40.19 \%$ \\
\hline 10 & 4.0 & PdiOAc) 12 & PtBU 3 HBF 4 & KOPIV & 3.14 & & 50-50 Toluene:lodobenzene & 14.73 & 105 & 24.0 & 1 & $19.00 \%$ \\
\hline 10 & 4.0 & PdiOACI2 12 & PtBu 3 HEF 4 & KOPIV & 3.00 & & 50:50 Dlowne:lodoberazene & 14.73 & 105 & 24.0 & $69.75 \%$ & $5.73 \%$ \\
\hline 10 & 4.0 & PdiOAci2 12 & PtBU 3 HEF 4 & KOPN & 3.00 & & 50:50 CPME lodabermene & 14.73 & 105 & 24.0 & $57.13 \%$ & $26.22 \%$ \\
\hline 10 & 4.0 & PdiOAci2 12 & PtBu 3 HEF 4 & KOPN & 3.00 & & $50.50 \mathrm{H}-\mathrm{MmOH}$ lodobenzene & 14.73 & 105 & 24.0 & $55.47 \%$ & $34.73 \%$ \\
\hline 10 & 4.0 & PdiOAc) /2 & PtBU 3 HEF 4 & KOPN & 3.57 & & 50.50 p-Xylene:lodoberzene & 14.73 & 105 & 24.0 & $72.15 \%$ & $14.59 \%$ \\
\hline 10 & 4.0 & PdiOacli2 & PtBu 3 HBF4 & KOPIV & 3.07 & & $50-50$ F Mtoluene: lodobenzene & 14.73 & 105 & 24.0 & $67.77 \%$ & $13.55 \%$ \\
\hline 10 & 4.0 & PवाORe/2 & & ROOPN & 328 & cuer2 & Sursup-xyenelosoberwere & 74.73 & 705 & 24.07 & $72.63 \%$ & $4.97 \%$ \\
\hline 10 & 4.0 & PdiOacil2 & & KOPN & 2.93 & $\mathrm{CuCl}_{2}$ & 5050 p-Xylene:lodoberzene & 14.73 & 105 & 24.0 & $71.31 \%$ & $6.00 \%$ \\
\hline 10 & 4.0 & PdiOacli2 & & KOPN & 2.93 & Cul & 50,50 p-Xylene:lodoberzene & 14.73 & 105 & 24.0 & $65.49 \%$ & $12.74 \%$ \\
\hline 10 & 4.0 & PdiOacil & & KOPN & 3.07 & cuca & 5050 p-xylene lodoberzene & 14.73 & 105 & 24.0 & $59.17 \%$ & $16.35 \%$ \\
\hline 10 & 4.0 & PdiOAc) 12 & & KOPN & 3.00 & $\infty$ & $50-50$ p-Xylene:lodobenzuene & 14.73 & 105 & 24.0 & $74.14 \%$ & $1.56 \%$ \\
\hline 10 & 4.0 & PdIOACI: 12 & & KOPIV & 2.79 & Cu(OAc)2 2 & $50-50$ p-Xylene:lodoberzzene & 14.73 & 105 & 24.0 & $6328 \%$ & $13.57 \%$ \\
\hline 10 & 4.0 & PdiOAci2 2 & & KOPN & 3.07 & & 5050 Toluere:lodobenzene & 14.73 & 105 & 24.0 & $74.12 \%$ & $0.00 \%$ \\
\hline 10 & 4.0 & PdiOAci2 & & KOPN & 3.14 & & 50:50 Dlowane:lodobenzene & 14.73 & 105 & 24.0 & $68.91 \%$ & $1.65 \%$ \\
\hline 10 & 4.0 & PdiOAci2 12 & & KOPIV & 3.07 & & 50:50 CPME lodaberzine & 14.73 & 105 & 24.0 & $76.83 \%$ & $0.51 \%$ \\
\hline 10 & 4.0 & PdiOAci2 12 & & KOPN & 3.00 & & $50.50 \mathrm{H}-\mathrm{AmOH}$ :lodobenzene & 14.73 & 105 & 24.0 & $67.96 \%$ & 0.0056 \\
\hline 10 & 4.0 & PdIOAC,12 & & KOPIV & 2.93 & & 5050 p-Xylene:lodoberzene & 14.73 & 105 & 24.0 & $77.52 \%$ & $1.39 \%$ \\
\hline 10 & 4.0 & PdiOacli & & KOPN & 3.28 & & $50-50$ F Holuere:lodobenzene & 14.73 & 105 & 24.0 & $72.99 \%$ & $0.13 \%$ \\
\hline 70 & & FवाOरC,12 & 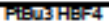 & RoOke & 3.36 & cuer2 & 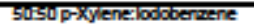 & 74.73 & 705 & 25.07 & $33.16 \%$ & $60.54 \%$ \\
\hline 10 & & PdiOAci2 12 & PtBU 3 HEF 4 & KONe & 2.85 & $\mathrm{CuCl} 2$ & 5050 p-Xylenelodoberzene & 14.73 & 105 & 24.0 & $46.75 \%$ & $40.01 \%$ \\
\hline 10 & & PdIOAC:12 & PtBU 3 HBF 4 & Kone & 3.26 & cul & $50-50$ p-xylenelodoberzene & 14.73 & 105 & 24.0 & $37.85 \%$ & $51.31 \%$ \\
\hline 10 & & PdiOACI2 & PtBu 3 HEF4 & KONC & 2.96 & cuca & 5050 p-Xylene:lodoberuzene & 14.73 & 105 & 24.0 & $54.93 \%$ & $3721 \%$ \\
\hline 10 & & PdiOAci2 12 & PtBU 3 HEF 4 & KONe & 2.56 & $a n$ & 5050 p-xylene lodoberzene & 14.73 & 105 & 24.0 & $58.99 \%$ & $28.80 \%$ \\
\hline 10 & & PdiOac)/2 & PtBU 3 HEF 4 & KONC & 3.16 & Curoac) 2 & $50-50$ p-Xylenelodoberzzene & 14.73 & 105 & 24.0 & $50.76 \%$ & $36.87 \%$ \\
\hline 10 & & PdiOAci2 2 & PtBU 3 HEF 4 & KONe & 3.06 & & 5050 p-xylene lodoberzene & 14.73 & 105 & 24.0 & $52.12 \%$ & $31.62 \%$ \\
\hline 10 & & PdIOAC, 2 & PtBu 3 HBF 4 & Kone & 3.16 & & 50:50 Dlowane:lodoberzene & 14.73 & 105 & 24.0 & $12.57 \%$ & $82.48 \%$ \\
\hline 10 & & PdiOAcli2 & PtBu 3 HBF 4 & KONe & 3.16 & & 50:50 CPME bodobermene & 14.73 & 105 & 24.0 & $36.62 \%$ & $49.42 \%$ \\
\hline 10 & & PdiOAcle & PtBU 3 HEF 4 & KONC & 3.06 & & $50.50 \mathrm{HAmOH}$ :lodobenzene & 14.73 & 105 & 24.0 & $14,47 \%$ & $80.29 \%$ \\
\hline 10 & & Pdionci2 & PtBu 3 HEF 4 & KONC & 3.26 & & $50-50$ p-Xylene:lodobe & 14.73 & 105 & 24.0 & $45.63 \%$ & $39.13 \%$ \\
\hline 10 & & PdiOAcl2 & $\mathrm{PtB} \cup 3 \mathrm{HBF} 4$ & KONC & 3.06 & & 50-50 FHoluere: iodobenzene & 14.73 & 105 & 24.0 & $33.59 \%$ & 34.0226 \\
\hline
\end{tabular}

\begin{tabular}{|c|c|c|c|c|c|c|c|c|c|c|c|c|}
\hline $\begin{array}{l}\text { su } \\
\text { emal }\end{array}$ & Name. & 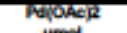 & Lyandico- & Conteyat & Nome & $=$ & Nemet & A & Temp & & Path & 310 \\
\hline 10 & & 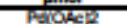 & & $\frac{\operatorname{mas} x}{\text { KOAC }}$ & $\frac{\text { Numb }}{275}$ & equat & 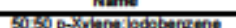 & 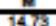 & $c$ & $\frac{10}{220}$ & $\sqrt{2} \times 20$ & \\
\hline 10 & & PAOREP 2 & & KOAC & 200 & CUCQ & $50.50 \mathrm{p}$-xylene lodoberzente & 1475 & 105 & 24.0 & $5221 \%$ & 340006 \\
\hline 10 & & Paloke 2 & & KOAC & 300 & Cul & $50.50 \mathrm{p}-x$, pene lodoberzene & 1475 & 105 & 24.0 & $30.75 \%$ & $5970 \%$ \\
\hline 10 & & 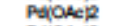 & & KOAC & 328 & Cuct & $50.50 \mathrm{p}-x$ y lene lodaberzene & 1473 & 105 & 24.0 & $5225 \%$ & $35.96 \%$ \\
\hline 10 & & Palokep & & KOAC & 200 & $\cos 20$ & $50.50 \mathrm{p}-x$ y lene lodoberzene & 1473 & 105 & 24.0 & $5060 \%$ & $28.16 \%$ \\
\hline 10 & & PAlokep 2 & & KOAC & 306 & $\mathrm{Cu}(\mathrm{CA}) \mathrm{C}_{2}$ & $50.50 \mathrm{p}-\mathrm{x}$ ylene lodaberzente & 1473 & 105 & 24.0 & $47.40 \%$ & $34.45 \%$ \\
\hline 10 & & Palorep & & KOAC & 200 & & 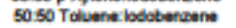 & 1475 & 105 & 24.0 & $5382 \%$ & $25.16 \%$ \\
\hline 10 & & Palokep & & KOAC & 200 & & 50.50 Dioxane ilodobercente & 1473 & 105 & 24.0 & 40.01\% & $35.02 \%$ \\
\hline 10 & & Palokep 2 & & KOAC & 285 & & 50.50 CPME lodotenzate & 1473 & 105 & 24.0 & $40.09 \%$ & $39.97 \%$ \\
\hline 10 & & Palokj2 & & KOAC & 336 & & 50.50 LAmOHtilodotensane & 1473 & 105 & 24.0 & $54.70 \%$ & $2281 \%$ \\
\hline 10 & & Plokez & & KOAC & 206 & & $50.50 \mathrm{p}-\mathrm{x}$ ylene:lodabercent & 1478 & 105 & 24.0 & $57.04 \%$ & 25.3046 \\
\hline 10 & & Pelaes 2 & & KOAC & 300 & & 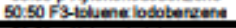 & 1475 & 105 & 24.0 & $44.51 \%$ & $27.026 \%$ \\
\hline 70 & 40 & PEORE? & & ROON & 3.43 & & 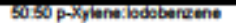 & 1475 & 105 & 240 & $7260 \%$ & $0.5 e n$ \\
\hline 10 & 40 & 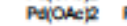 & PABUS HBF 4 & KOPN & 3.14 & & $50.50 \mathrm{p}-x$ y lene ledaberzent & 1473 & 105 & 24.0 & $5820 \%$ & $27.10 \%$ \\
\hline 10 & 4.0 & Polokep & & KOAC & 3.16 & & $5050 \mathrm{p}-x_{\text {y lene iodaberzene }}$ & 1473 & 105 & 24.0 & $50.75 \%$ & $37.95 \%$ \\
\hline 10 & 4.0 & Paloke12 & PBDUS HBF 4 & $\mathrm{KHCOO}$ & 3.10 & TFA & $50.50 \mathrm{p}$-Xylene lodaborsene & 1475 & 105 & 24.0 & $55.77 \%$ & $30.38 \%$ \\
\hline 10 & 4.0 & Palokep & PABUS HBF 4 & $\mathrm{KHCOS}$ & 280 & PIOOH & $50.50 \mathrm{p}$ - Xylene lodabersene & 1473 & 105 & 24.0 & $58.65 \%$ & 22194 \\
\hline 10 & 4.0 & Paloepla & PABUS HBF 4 & $\mathrm{KHCOO}$ & 320 & $\mathrm{ACOH}$ & $50.50 \mathrm{p}$-X́plene lodaberzene & 1475 & 105 & 24.0 & $55.51 \%$ & $31.85 \%$ \\
\hline 10 & 4.0 & Pdiopivg & & KOPN & 300 & & $5050 \mathrm{p}$-Xylenelodoberzene & 1473 & 105 & 24.0 & $00.09 \%$ & 03866 \\
\hline 10 & 4.0 & PdiOPAVR & PABUS HBF 4 & KOPA & 300 & & $50.50 \mathrm{p}-\mathrm{x}$ ylene lodabercene & 1473 & 105 & 24.0 & $74.56 \%$ & $4.10 \%$ \\
\hline 10 & 40 & PAiOPANR & & KOAC & 300 & & 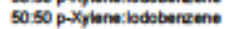 & 1475 & 105 & 24.0 & $58.75 \%$ & $3209 \%$ \\
\hline 10 & 4.0 & Paloké2 & & $\mathrm{KHCOO}$ & 300 & TFA & $50.50 \mathrm{p}$ - Xylane lodaberzene & 1475 & 105 & 24.0 & $77.41 \%$ & $0.27 \%$ \\
\hline 10 & 4.0 & Paloke? & & KHCOS & 280 & PhoOH & $50.50 \mathrm{p}-x$ y lene lodaberzent & 1473 & 105 & 24.0 & 56.15\% & $0.11 \%$ \\
\hline 10 & 4.0 & PAOAR2 & & $\mathrm{KHCOS}$ & 3.40 & $\mathrm{AOOH}$ & $50.50 \mathrm{p}-\mathrm{X}$ y lene iodaberzene & 1475 & 105 & 24.0 & $75.52 \% 6$ & $0.00 \%$ \\
\hline 70 & 40 & PQRाFमश2 & PABख्यामहन & ROOW & 235 & & 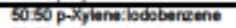 & 1475 & 705 & 24.0 & $5722 \%$ & $30.00 \%$ \\
\hline 10 & 4.0 & PQTTFA:2 & & KOP & 321 & & $50.50 \mathrm{p}$-Xylene lodaberzente & 1475 & 105 & 24.0 & $51.35 \%$ & $35.11 \%$ \\
\hline 10 & 40 & PdTFAP2 & & KOAC & 300 & & $50.50 \mathrm{p}$ - Xylene.lodaberzene & 1473 & 105 & 24.0 & $51.20 \%$ & $3001 \%$ \\
\hline 10 & 4.0 & $P B(O R=12$ & PABUS HBF 4 & $\mathrm{k} 2 \mathrm{cos}$ & 1930 & TFA & $67.35 \mathrm{p}$-Xýlene lodabercene & 2210 & 105 & 24.0 & $90.78 \%$ & 30226 \\
\hline 10 & 40 & PAORe12 & PABUS HEF 4 & $\mathrm{~K} 2 \mathrm{COOS}$ & 201 & PAOH & $67.35 \mathrm{p}-$-Xylene.lodoberzene & 2210 & 105 & 24.0 & $95.6 e \%$ & $30.63 \%$ \\
\hline 10 & 4.0 & Palokep & PABUS HBF 4 & $\mathrm{k} 2 \mathrm{COOS}$ & 1006 & $\mathrm{~A}=\mathrm{OH}$ & $50.50 \mathrm{p}$-Xylene lodaberzene & 1475 & 105 & 24.0 & $24.62 \%$ & $5482 \%$ \\
\hline 10 & 4.0 & 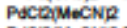 & & KOPA & 300 & & $50.50 \mathrm{p}-x$ y lene lodaberzente & 1473 & 105 & 24.0 & $78.19 \%$ & 21936 \\
\hline 10 & 40 & PACESMeCNIR & PQUS HBF 4 & KOPF & 3.43 & & $50.50 \mathrm{p}$-Xylene lodoberzente & 1473 & 105 & 24.0 & $47.50 \%$ & $41.50 \%$ \\
\hline 10 & 4.0 & PACESMeCNp & & KOAC & 285 & & $50.50 \mathrm{p}-\mathrm{x}$ ylene lodabercene & 1475 & 105 & 24.0 & $48.97 \%$ & $30.44 \%$ \\
\hline 10 & 4.0 & Palakep & & $\mathrm{k} 2 \mathrm{cos}$ & 832 & TFA & $50.50 \mathrm{p}-x$ y lene lodaberzene & 1475 & 105 & 24.0 & $51.63 \%$ & $0.00 \%$ \\
\hline 10 & 40 & Parep? & & $\mathrm{K} 2 \mathrm{COOS}$ & 9.10 & PAOH & $5050 \mathrm{p}-x$ ylene lodaberzente & 1473 & 105 & 240 & $34.51 \%$ & 0.056 \\
\hline 10 & 4.0 & Pulake 2 & & $\mathrm{k} 200 \mathrm{cos}$ & 781 & $\mathrm{AOOH}$ & 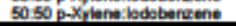 & 0.10 & 105 & 24.0 & $34.00 \%$ & 0.186 \\
\hline
\end{tabular}


80 een $* 3$

\begin{tabular}{|c|c|c|c|c|c|c|c|c|c|c|c|c|}
\hline ar & איה & & $\begin{array}{l}\text { robitivence } \\
\text { Name }\end{array}$ & ands & & & Nome & & remp & & & \\
\hline Flus & & $510 \%$ & & & Teorents & 3,45 & bionese & 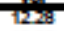 & & & 48.6556 & 45:1:95: \\
\hline 10 & 10.0 & $5.0 \%$ & & & KOPN & 3.07 & CPME & 12.28 & 118 & 240 & $37.45 \%$ & $59.22 \%$ \\
\hline 10 & 10.0 & 5.036 & & & KOPIV & 293 & $\mathrm{HAMOH}$ & 12.28 & 118 & 240 & $29.73 \%$ & 0.65455 \\
\hline 10 & 10.0 & $5.0 \%$ & & & KOPN & 3.64 & p-xylene & 12.28 & 118 & 240 & $52.32 \%$ & 0.46198 \\
\hline 10 & 10.0 & $5.0 \%$ & & & KOPN & 3.07 & Fotbluene & 12.28 & 118 & 240 & $52.23 \%$ & 0.44475 \\
\hline 10 & 10.0 & $5.0 \%$ & & & KOPN & 293 & DaMN & 12.28 & 118 & 240 & 0 & $84.10 \%$ \\
\hline 10 & & $5.0 \% 6$ & & & KOPN & 364 & 50.50 Dloxane Eromoberwere & 14.73 & 118 & 240 & 0 & $98.62 \%$ \\
\hline 10 & & $5.0 \%$ & & & KOPN & 293 & 50:50 CPME:Bromoberzene & 14.73 & 118 & 240 & $0.79 \%$ & $96.39 \%$ \\
\hline 10 & & $5.0 \%$ & & & KOPN & 293 & $50: 50 \mathrm{tAm}$ CH:Bromoberzene & 14.73 & 118 & 240 & $0.21 \%$ & $9794 \%$ \\
\hline 10 & & $5.0 \%$ & & & KOPIN & 307 & 50.50 p-xylene Bromaberwene & 14.73 & 118 & 240 & $229 \%$ & $93.42 \%$ \\
\hline 10 & & $5.0 \%$ & & & KOPN & 307 & $50: 50$ foroluene: Bromoberaxene & 14.73 & 118 & 240 & $296 \%$ & $9238 \%$ \\
\hline 10 & & $5.0 \%$ & & & KOPN & 3.07 & 50.50 DM MCEromabermene & 1473 & 118 & 240 & $0.05 \%$ & $92.75 \%$ \\
\hline Fiv & Futu & $50 \times 5$ & FINOA & Flub: & Refocos & 250 & bionsere & 1228 & 1718 & 220 & $38.49 \%$ & $6003 \%$ \\
\hline 10 & 10.0 & $5.0 \%$ & PNOH & $100 \%$ & $\mathrm{NHCOS}$ & 290 & CPME & 1228 & 118 & 240 & $30.17 \%$ & $6905 \%$ \\
\hline 10 & 10.0 & $5.0 \% 6$ & PNOH & $10.0 \%$ & $\mathrm{NHCO3}$ & 290 & $\mathrm{MAmOH}$ & 1228 & 118 & 240 & $65.53 \%$ & $32.14 \%$ \\
\hline 10 & 10.0 & $5.0 \%$ & PMOH & $10.0 \%$ & $\mathrm{NHCO3}$ & 3,40 & p-xylene & 12.28 & 118 & 240 & $64.09 \%$ & $34.15 \%$ \\
\hline 10 & 10.0 & $50 \%$ & PNOH & $10.0 \%$ & $\mathrm{NHCO3}$ & 330 & Fitoluene & 12.28 & 118 & 240 & $61.69 \%$ & $36.89 \%$ \\
\hline 10 & 10.0 & $5.0 \% 6$ & PNOH & $10.0 \%$ & $\mathrm{NHCO3}$ & 3.10 & DaMc & 1228 & 118 & 240 & $1.55 \%$ & $8330 \%$ \\
\hline 10 & & $5.0 \%$ & PNOH & $10.0 \%$ & $\mathrm{KHCO3}$ & 290 & 50.50 Dioxane Promobermene & 14.73 & 118 & 240 & $0.18 \%$ & $98.64 \%$ \\
\hline 10 & & $5.0 \%$ & PMOH & $10.0 \%$ & $\mathrm{NHCO3}$ & 280 & 50:50 CPME:Bromoberzzene & 14.73 & 118 & 240 & $0.10 \%$ & $99.12 \%$ \\
\hline 10 & & 5.066 & PNOH & $10.0 \%$ & $\mathrm{NHCOS}$ & 2.70 & 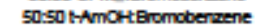 & 14.73 & 118 & 240 & $0.21 \%$ & $97.79 \%$ \\
\hline 10 & & $5.0 \%$ & PNOH & $10.0 \%$ & $\mathrm{NHCOS}$ & 3.10 & $50-50$ p-xylene Eromaberimente & 14.73 & 118 & 240 & $0.27 \%$ & $97.73 \%$ \\
\hline 10 & & $5.0 \%$ & PMOH & $10.0 \%$ & $\mathrm{KHCO}$ & 3.20 & $50: 50$ f Holuene:Bromoberzene & 14.73 & 118 & 240 & 0.45\% & $98.38 \%$ \\
\hline 10 & & $5.0 \%$ & PNOH & $100 \%$ & $\mathrm{KHCO3}$ & 300 & 50.50 DMACBromabermene & 14.73 & 118 & 240 & $0.07 \%$ & $95.78 \%$ \\
\hline 70 & 70.0 & 5005 & 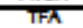 & Flubs & RAcos & 250 & Diarare & 7228 & 716 & $2 \pi 0$ & $79.84 \%$ & $17.93 \%$ \\
\hline 10 & 10.0 & $5.0 \%$ & TFA & $10.0 \%$ & $\mathrm{NHCOS}$ & 290 & CPME & 12.28 & 118 & 240 & 48.3746 & $50.63 \%$ \\
\hline 10 & 10.0 & $5.0 \%$ & TFA & $10.0 \%$ & $\mathrm{NHCO3}$ & 390 & $\mathrm{MMOH}$ & 12.28 & 118 & 240 & $51.19 \%$ & $47.05 \%$ \\
\hline 10 & 10.0 & $5.0 \%$ & TFA & $10.0 \%$ & $\mathrm{NHCO3}$ & 300 & p-xylene & 12.28 & 118 & 240 & $62.74 \%$ & $3454 \%$ \\
\hline 10 & 10.0 & $5.0 \% 6$ & TFA & $10.0 \%$ & $\mathrm{NHCO3}$ & 300 & FHtoluene & 1228 & 118 & 240 & $63.39 \%$ & $35.20 \%$ \\
\hline 10 & 10.0 & $5.0 \%$ & TFA & $10.0 \%$ & $\mathrm{NHCO3}$ & 300 & Danc & 12.28 & 118 & 240 & $1.33 \%$ & $80.37 \%$ \\
\hline 10 & & $5.0 \%$ & TFA & $10.0 \%$ & $\mathrm{NHCOS}$ & 3.20 & 50.50 Dloxane Promabermene & 14.73 & 118 & 240 & $0.18 \%$ & $98.60 \%$ \\
\hline 10 & & $5.0 \% 6$ & TFA & $10.0 \%$ & $\mathrm{NHCO3}$ & 280 & 50:50 CPME:Bromoberziene & 14.73 & 118 & 240 & $0.12 \%$ & $9901 \%$ \\
\hline 10 & & $5.0 \%$ & TFA & $100 \%$ & $\mathrm{NHCOS}$ & 290 & $50.50 \mathrm{MAmOH}$ Bromoberwene & 14.73 & 118 & 240 & $0.39 \%$ & $9799 \%$ \\
\hline 10 & & $5.0 \% 6$ & TFA & $10.0 \%$ & $\mathrm{KHCOS}$ & 290 & $50-50$ p-xylene Eromaberizene & 14.73 & 118 & 240 & $0.47 \%$ & $9805 \%$ \\
\hline $\begin{array}{l}10 \\
10\end{array}$ & & $\begin{array}{l}5.0 \% 6 \\
5.0 \% 6\end{array}$ & TFA & $\begin{array}{l}10.0 \% \\
10.0 \%\end{array}$ & $\begin{array}{l}\text { NOCOS } \\
\text { NHCO3 }\end{array}$ & $\begin{array}{r}320 \\
300\end{array}$ & 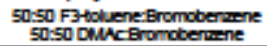 & $\begin{array}{l}14.73 \\
14.73\end{array}$ & $\begin{array}{l}118 \\
118\end{array}$ & 240 & $\begin{array}{l}0.69 \% 6 \\
0.10 \% 6\end{array}$ & $\begin{array}{l}96.67 \% \\
96.87 \%\end{array}$ \\
\hline 70 & 70.00 & $50 \%$ & REOH & Folos & Retoos & 250 & Diaxpe & 1228 & Tils & 220 & $77.39 \mathrm{~m}$ & $20.31 \%$ \\
\hline 10 & 10.0 & $5.0 \%$ & $\mathrm{NCOH}$ & $10.0 \%$ & $\mathrm{NHCO3}$ & 3.10 & CPME & 12.28 & 118 & 240 & $43.92 \%$ & $5484 \%$ \\
\hline 10 & 10.0 & $50 \%$ & $\mathrm{ACOH}$ & $10.0 \%$ & $\mathrm{NHCO3}$ & 3.10 & $\mathrm{HAMOH}$ & 12.28 & 118 & 240 & 57.3996 & $39.77 \%$ \\
\hline 10 & 10.0 & $5.0 \% 6$ & $\mathrm{ACOH}$ & $10.0 \%$ & $\mathrm{NHCO3}$ & 290 & p-xylene & 12.28 & 118 & 240 & $65.27 \%$ & $3272 \%$ \\
\hline 10 & 10.0 & $5.0 \%$ & $\mathrm{ACOH}$ & $10.0 \%$ & $\mathrm{NHCO3}$ & 3.20 & F-toluene & 1228 & 118 & 240 & $66.21 \%$ & $32.36 \%$ \\
\hline 10 & 10.0 & $5.0 \% 6$ & $\mathrm{ACOH}$ & $10.0 \%$ & $\mathrm{NHCOS}$ & 300 & DaAk & 1228 & 118 & 240 & $1.34 \%$ & $7909 \%$ \\
\hline 10 & & $5.0 \%$ & $\mathrm{ACOH}$ & $10.0 \%$ & $\mathrm{NHCO3}$ & 3.00 & 50.50 Dloxane Promabenzere & 14.73 & 118 & 240 & $0.17 \%$ & $98.63 \%$ \\
\hline 10 & & 5006 & $\mathrm{ACOH}$ & $10.0 \%$ & $\mathrm{NHCO3}$ & 3.30 & 50:50 CPME:Bromoberzene & 14.73 & 118 & 240 & $0.16 \%$ & $98.55 \%$ \\
\hline 10 & & $5.0 \%$ & $\mathrm{NCOH}$ & $10.0 \%$ & $\mathrm{NHCO3}$ & 280 & $50: 50 \mathrm{Am}$ OHtBromobersuene & 14.73 & 118 & 240 & $0.33 \%$ & $97.89 \%$ \\
\hline 10 & & $5.0 \%$ & $\mathrm{ACOH}$ & $10.0 \%$ & $\mathrm{NHCO3}$ & 290 & 50.50 p-xylene Bromaberimene & 14.73 & 118 & 240 & $1.18 \%$ & $95.80 \%$ \\
\hline $\begin{array}{l}10 \\
10\end{array}$ & & $\begin{array}{l}5.056 \\
5.0 \% 6\end{array}$ & $\mathrm{ACOH}$ & $\begin{array}{l}10.0 \% \\
10.0 \%\end{array}$ & $\begin{array}{l}\mathrm{NOHCO} 3 \\
\mathrm{NHCO} 3\end{array}$ & $\begin{array}{l}290 \\
320\end{array}$ & $\begin{array}{l}50: 50 \text { FHoluene:Bromoberzene } \\
50.50 \text { DM }\end{array}$ & 1473 & $\begin{array}{l}118 \\
118\end{array}$ & 240 & $\begin{array}{l}0.59 \% \\
0.09 \%\end{array}$ & $97.55 \%$ \\
\hline & & & & & & & & 14.73 & 118 & 200 & $0.08 \%$ & \\
\hline
\end{tabular}




\begin{tabular}{|c|c|c|c|c|c|c|c|c|c|c|c|c|}
\hline $\mathrm{mol}$ & Pat & (1) & Arobitiverch & $\operatorname{cosayat}$ & Nome & Eoutv & Name & & Iming & & $\begin{array}{l}\text { Put? } \\
A P\end{array}$ & $\sin _{A P}$ \\
\hline 10 & fout & 5050 & cuetr & folus & Rorent & sor & Dxiyene & 7223 & $78 \mathrm{~s}$ & 241 & $2 \pi 22+\%$ & Gr:tas\% \\
\hline 10 & 10.0 & $5.0 \%$ & Gen 2 & $10.0 \%$ & KOPN & 3.00 & p-xylene & 12.28 & 118 & 240 & $51.44 \%$ & $38.49 \%$ \\
\hline 10 & 10.0 & $5.0 \%$ & CuCL2 & $10.0 \%$ & KOPNV & 3.00 & CPME & 12.28 & 118 & 240 & $23.49 \%$ & $\pi .66 \%$ \\
\hline 10 & 10.0 & $5.0 \%$ & cuen & $10.0 \%$ & KOPN & 3.07 & CPME & 12.28 & 118 & 240 & $21.54 \%$ & $72.65 \%$ \\
\hline 10 & 100 & $5.0 \%$ & CuCD2 & $10.0 \%$ & KOPNV & 3.07 & $\mathrm{M} M \mathrm{MCH}$ & 12.28 & 118 & 240 & $18.39 \%$ & $68.10 \%$ \\
\hline 10 & 10.0 & $5.0 \%$ & aen 2 & $10.0 \%$ & KOPN & 2.93 & $\mathrm{MmOH}$ & 12.28 & 118 & 240 & $20.04 \%$ & $\pi 0.05 \%$ \\
\hline 10 & & $5.0 \%$ & CuCl2 & $10.0 \%$ & KOPN & 2.93 & 50.50 p-xyene:Bromoberusene & 14.73 & 118 & 240 & $0.08 \%$ & $89.68 \%$ \\
\hline 10 & & $5.0 \%$ & aen 2 & $10.0 \%$ & KOPN & 3.07 & soso p-xyene:Bromoberzene & 14.73 & 118 & 240 & $0.05 \%$ & $73.12 \%$ \\
\hline 10 & & $5.0 \%$ & CuCL2 & $10.0 \%$ & KOPNV & 3.14 & 50:50 cPME :Bromoberwene & 14.73 & 118 & 240 & $0.05 \%$ & $86.64 \%$ \\
\hline 10 & & $5.0 \%$ & aen & $10.0 \%$ & KOPN & 2.93 & 50:50 CPME Bromobenture & 14.73 & 118 & 240 & $0.09 \%$ & $68.97 \%$ \\
\hline 10 & & $5.0 \%$ & Cuci2 & $10.0 \%$ & KOPN & 3.21 & $5050 \mathrm{t}-\mathrm{MmCH}$ :Eromoberzene & 14.73 & 118 & 240 & $0.05 \%$ & $83.96 \%$ \\
\hline 10 & & $5.0 \%$ & aen 2 & 10.05 & KOPIV & 3.14 & $5050 \mathrm{~A}$ AmOH:Eromoberzene & 14.73 & 118 & 240 & $0.05 \%$ & $73.37 \%$ \\
\hline 10 & Futo & 5055 & $\cos 20^{\circ}$ & Folus & ReOPN & 3.14 & p-xylene & 72283 & 178 & 240 & $25.29 \%$ & $71.17 \%$ \\
\hline 10 & 100 & $5.0 \%$ & CU(OAC) & $10.0 \%$ & KOPN & 2.93 & p-xylene & 12.28 & 118 & 240 & $59.96 \%$ & $29.16 \%$ \\
\hline 10 & 10.0 & $5.0 \%$ & ano & $10.0 \%$ & KOPN & 3.00 & CPME & 12.28 & 118 & 240 & $22.93 \%$ & 74. $45 \%$ \\
\hline 10 & 10.0 & $5.0 \%$ & CUIOAC) & $10.0 \%$ & KOPIV & 2.93 & CPME & 12.28 & 118 & 240 & $16.13 \%$ & $79.22 \% 6$ \\
\hline 10 & 10.0 & $5.0 \%$ & $a, 0$ & $10.0 \%$ & KOPN & 2.93 & $\mathrm{M} M \mathrm{MH}$ & 12.28 & 118 & 240 & $21.31 \%$ & $68.35 \%$ \\
\hline 10 & 10.0 & $5.0 \%$ & CulOAC) & $10.0 \%$ & KOPN & 3.00 & $\mathrm{MMMOH}$ & 12.28 & 118 & 240 & $20.03 \%$ & $66.33 \%$ \\
\hline 10 & & $5.0 \%$ & 0,00 & $10.0 \%$ & KOPN & 2.93 & soso p-xylene:Bromoberixene & 14.73 & 118 & 240 & $0.04 \%$ & $95.02 \%$ \\
\hline 10 & & $5.05 \%$ & CUIOAC) & $10.0 \%$ & KOPN & 3.86 & 50 so p-xyene:Bromoberizene & 14.73 & 118 & 240 & $0.05 \%$ & $87.73 \%$ \\
\hline 10 & & $5.0 \%$ & ano & $10.0 \%$ & KOPIV & 3.00 & 50:50 CPME Bromobenture & 14.73 & 118 & 240 & $0.08 \%$ & $87.85 \%$ \\
\hline 10 & & $5.0 \%$ & CulOAC) & $10.0 \%$ & KOPN & 3.07 & 50:50 cPME Bromobervene & 14.73 & 118 & 240 & $0.05 \%$ & $83.50 \%$ \\
\hline 10 & & $5.0 \%$ & ano & $10.0 \%$ & KOPNV & 2.93 & $5050 \mathrm{MAmCH}:$ Bromoberzene & 14.73 & 118 & 240 & $0.29 \%$ & $93.70 \% 6$ \\
\hline 10 & & $5.0 \%$ & $\mathrm{Cu}(\mathrm{ONC})$ & $10.0 \%$ & KOPN & 3.28 & $50.50 \mathrm{t}-\mathrm{MmOH}:$ Bromoberzene & 14.73 & 118 & 240 & $0.04 \%$ & $85.09 \%$ \\
\hline $\begin{array}{l}70 \\
10\end{array}$ & $\begin{array}{l}7010 \\
10.0\end{array}$ & $\begin{array}{l}510 \% 5 \\
5.0 \%\end{array}$ & culo & $\begin{array}{l}70.0 \% 5 \\
10.05 \%\end{array}$ & $\begin{array}{l}\text { ROOFN } \\
\text { KOPNV }\end{array}$ & $\begin{array}{l}300 \\
2.86\end{array}$ & $\begin{array}{l}\text { p-xylene } \\
\text { p-xylene }\end{array}$ & $\frac{7228}{12.28}$ & $\begin{array}{l}718 \\
118\end{array}$ & $\begin{array}{l}2210 \\
240\end{array}$ & $\begin{array}{l}43.01 \% \\
16.67 \%\end{array}$ & $\frac{46.39 \%}{78.28 \%}$ \\
\hline 10 & 10.0 & $5.0 \%$ & aud & $10.0 \%$ & KOPlV & 3.21 & CPME & 12.28 & 118 & 240 & $21.62 \%$ & $75.00 \%$ \\
\hline 10 & 10.0 & $5.0 \%$ & cuo & $10.0 \%$ & KOPN & 2.93 & CPME & 12.28 & 118 & 240 & $21.45 \%$ & $69.34 \%$ \\
\hline 10 & 10.0 & $5.0 \%$ & cucl & $10.0 \%$ & KOPNV & 3.14 & $\mathrm{MmOH}$ & 12.28 & 118 & 240 & $16.39 \%$ & $71.24 \%$ \\
\hline 10 & 10.0 & $5.0 \%$ & cuo & $10.0 \%$ & KOPN & 2.93 & $\mathrm{MmOH}$ & 12.28 & 118 & 240 & $16.89 \%$ & 69.4096 \\
\hline 10 & & $5.0 \%$ & and & $10.0 \%$ & KOPN & 2.93 & $50.50 \mathrm{p}-\mathrm{x}$ /ene:Bromoberizene & 14.73 & 118 & 240 & $0.21 \%$ & $74.35 \%$ \\
\hline 10 & & $5.0 \%$ & cuo & $10.0 \%$ & KOPNV & 3.07 & $50.50 \mathrm{p}$-Xilene:Bromoberume & 14.73 & 118 & 240 & $0.05 \%$ & $75.14 \%$ \\
\hline 10 & & $5.0 \%$ & cucl & $10.0 \%$ & KOPN & 0.00 & 50:50 crim E Bromobenvene & 14.73 & 118 & 240 & $0.07 \%$ & $64.52 \%$ \\
\hline 10 & & $5.0 \%$ & cuo & $10.0 \%$ & KOPN & 3.14 & 50:50 CPME Bromobenzere & 14.73 & 118 & 240 & $0.07 \%$ & $72.51 \%$ \\
\hline $\begin{array}{l}10 \\
10\end{array}$ & & $\begin{array}{l}50 \% \\
5.0 \%\end{array}$ & $\begin{array}{l}\text { cucl } \\
\text { cuo }\end{array}$ & $\begin{array}{l}10.0 \% \\
10.0 \%\end{array}$ & $\begin{array}{l}\text { KOPN } \\
\text { KOPN }\end{array}$ & $\begin{array}{l}3.28 \\
2.86\end{array}$ & 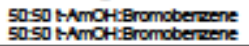 & $\begin{array}{l}14.73 \\
14.73\end{array}$ & $\begin{array}{l}118 \\
118\end{array}$ & 240 & $0.05 \%$ & $\frac{86.7226}{75.73 \%}$ \\
\hline 10 & 700 & 5056 & 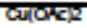 & 70105 & ROOPNV & 2.95 & p-xylene & 7223 & 718 & 2210 & $31.32 \%$ & $60.64 \%$ \\
\hline 10 & 10.0 & $5.0 \%$ & & $10.0 \%$ & KOPNV & 3.07 & p-xylene & 12.28 & 118 & 240 & $29,43 \%$ & $66.75 \%$ \\
\hline 10 & 100 & $5.0 \%$ & CulOak) $)_{2}$ & $10.0 \%$ & KOPN & 3.00 & CPME & 12.28 & 118 & 240 & $19.38 \%$ & $72.70 \%$ \\
\hline 10 & 10.0 & $5.0 \%$ & $\mathrm{Cu}$ & $10.0 \%$ & KOPN & 3.00 & CPME & 12.28 & 118 & 240 & $19.57 \%$ & $73.31 \%$ \\
\hline 10 & 10.0 & $5.0 \%$ & CulONC)2 & $10.0 \%$ & KOPN & 3.00 & $\mathrm{MMMOH}$ & 12.28 & 118 & 240 & $17.91 \%$ & $68.85 \%$ \\
\hline 10 & 10.0 & $5.0 \%$ & $\mathrm{Cu}$ & $10.0 \%$ & KOPN & 3.36 & $\mathrm{MMNOH}$ & 12.28 & 118 & 240 & $10.55 \%$ & $81.25 \%$ \\
\hline 10 & & $5.0 \%$ & Culokcle & $10.0 \%$ & KOPN & 3.14 & $50.50 \mathrm{p}-\mathrm{x} / \mathrm{ene}$ :Bromoberziene & 14.73 & 118 & 240 & $0.18 \%$ & $77.58 \%$ \\
\hline 10 & & $50 \%$ & cul & $10.0 \%$ & KOPN & 2.93 & $50: 50$ p-xylene:Bromoberuene & 14.73 & 118 & 240 & $0.08 \%$ & $82.87 \%$ \\
\hline 10 & & $5.0 \%$ & CulOACl2 & $10.0 \%$ & KOPN & 3.00 & 50:50 CPME Bromobentere & 14.73 & 118 & 240 & $0.05 \%$ & $80.75 \%$ \\
\hline 10 & & $5.0 \%$ & Cull & $10.0 \%$ & KOPN & 3.00 & 50:50 CPME Bromobervere & 14.73 & 118 & 240 & $0.05 \%$ & $83.89 \%$ \\
\hline 10 & & $\begin{array}{l}50 \% \\
500 \%\end{array}$ & CulOAC:2 & $10.0 \%$ & KOPN & 3.14 & 5050 I-AmOH:Bromoberzene & 14.73 & 118 & 240 & $0.04 \%$ & $80.61 \%$ \\
\hline
\end{tabular}




\begin{tabular}{|c|c|c|c|c|c|c|c|c|c|c|c|}
\hline $\begin{array}{c}\text { DOE \#1 } \\
\text { Pd } \\
\text { source }\end{array}$ & Base & solvent & Time & Phl equlv & Pd mol \% & $\begin{array}{l}\text { base } \\
\text { equiv }\end{array}$ & $\begin{array}{l}\text { HOAC } \\
\text { equilv }\end{array}$ & Vol & Temp & $\begin{array}{c}\text { AP } \\
\text { Product }\end{array}$ & AP SM \\
\hline$P d(O A C) 2$ & KAHCOS & Dioxane & 24 & 2 & 3 & 1.97 & 0.3 & 6 & 80 & 40.0451 & 55.04 \\
\hline $\mathrm{Pd}(\mathrm{OAC}) 2$ & $\mathrm{KHCO} 3$ & Dioxane & 24 & 2 & 3 & 2.01 & 0.1 & 12 & 80 & 25.677 & 70.17 \\
\hline $\mathrm{Pd}(\mathrm{OAC}) 2$ & $\mathrm{KHCO} 3$ & Dloxane & 24 & 2 & 3 & 2.95 & 0.1 & 6 & 80 & 30.999 & 64.517 \\
\hline $\mathrm{Pd}(\mathrm{OAC}) 2$ & $\mathrm{KHCO} 3$ & Dioxane & 24 & 2 & 3 & 3.03 & 0.3 & 12 & 80 & 24.243 & 72.286 \\
\hline$P d(O A C) 2$ & KHCO3 & Dloxane & 24 & 10 & 3 & 2.03 & 0.1 & 6 & 80 & 93.079 & 0 \\
\hline Pd(OAC)2 & KHCO3 & Dloxane & 24 & 10 & 3 & 1.97 & 0.3 & 12 & 80 & 93.915 & 0 \\
\hline $\mathrm{Pd}\left(\mathrm{OAC}_{\mathrm{C}}\right) 2$ & KHCO3 & Dloxane & 24 & 10 & 3 & 3.02 & 0.3 & 6 & 80 & 93.603 & 0 \\
\hline $\mathrm{Pd}(O A C) 2$ & KHCO3 & Dloxane & 24 & 10 & 3 & 2.96 & 0.1 & 12 & 80 & 92.35 & 0 \\
\hline $\mathrm{Pd}(\mathrm{OAC}) 2$ & KHCO3 & Dloxane & 24 & 6 & 5 & 2.53 & 0.2 & 9 & 80 & 91.871 & 0.17 \\
\hline $\mathrm{Pd}(\mathrm{OAC}) 2$ & KHCO3 & Dloxane & 24 & 2 & 7 & 2.66 & 0.1 & 6 & 80 & 42.568 & 51.857 \\
\hline $\mathrm{Pd}(\mathrm{OAC}) 2$ & KHCO3 & Dloxane & 24 & 2 & 7 & 2.07 & 0.3 & 12 & 80 & 29.339 & 66.833 \\
\hline $\mathrm{Pd}(\mathrm{OAC}) 2$ & $\mathrm{KHCO3}$ & Dloxane & 24 & 2 & 7 & 3.01 & 0.3 & 6 & 80 & 48.072 & 41.328 \\
\hline $\mathrm{Pd}(O A C) 2$ & KHCO3 & Dloxane & 24 & 2 & 7 & 3.00 & 0.1 & 12 & 80 & 39.851 & 53.511 \\
\hline $\mathrm{Pd}(O A C) 2$ & KHCO3 & Dloxane & 24 & 10 & 7 & 2.00 & 0.3 & 6 & 80 & 87.679 & 0 \\
\hline $\mathrm{Pd}\left(\mathrm{OAC}_{\mathrm{C}}\right) 2$ & KHCO3 & Dloxane & 24 & 10 & 7 & 1.93 & 0.1 & 12 & 80 & 86.655 & 0 \\
\hline $\mathrm{Pd}(\mathrm{OAC}) 2$ & KHCO3 & Dloxane & 24 & 10 & 7 & 3.00 & 0.1 & 6 & 80 & 89.423 & 0 \\
\hline $\mathrm{Pd}(\mathrm{OAC}) 2$ & KHCO3 & Dloxane & 24 & 10 & 7 & 3.03 & 0.3 & 12 & 80 & 85.736 & 0 \\
\hline $\mathrm{Pd}(O A C) 2$ & KHCO3 & Dloxane & 24 & 6 & 3 & 2.45 & 0.2 & 9 & 90 & 83.364 & 11.666 \\
\hline $\mathrm{Pd}(\mathrm{OAC}) 2$ & KHCO3 & Dioxane & 24 & 2 & 5 & 2.44 & 0.2 & 9 & 90 & 31.998 & 63.183 \\
\hline $\mathrm{Pd}(\mathrm{OAC}) 2$ & KHCO3 & Dloxane & 24 & 6 & 5 & 1.84 & 0.2 & 9 & 90 & 82.94 & 10.658 \\
\hline $\mathrm{Pd}(\mathrm{OAC}) 2$ & $\mathrm{KHCO}$ & Dloxane & 24 & 6 & 5 & 2.35 & 0.2 & 6 & 90 & 92.606 & 0.046 \\
\hline $\mathrm{Pd}(O A C) 2$ & KHCO3 & Dloxane & 24 & 6 & 5 & 2.54 & 0.1 & 9 & 90 & 91.266 & 0 \\
\hline $\mathrm{Pd}(O A C) 2$ & KHCO3 & Dloxane & 24 & 6 & 5 & 2.48 & 0.2 & 9 & 90 & 83.287 & 10.685 \\
\hline $\mathrm{Pd}(\mathrm{OAC}) 2$ & KHCO3 & Dloxane & 24 & 6 & 5 & 2.51 & 0.2 & 9 & 90 & 86.34 & 7.106 \\
\hline $\mathrm{Pd}(\mathrm{OAC}) 2$ & KHCO3 & Dloxane & 24 & 6 & 5 & 2.42 & 0.2 & 9 & 90 & 91.353 & 0.987 \\
\hline $\mathrm{Pd}\left(\mathrm{OAC}_{\mathrm{C}}\right) 2$ & KHCO3 & Dloxane & 24 & 6 & 5 & 2.42 & 0.2 & 9 & 90 & 81.405 & 12.366 \\
\hline $\mathrm{Pd}(O A C) 2$ & KHCO3 & Dloxane & 24 & 6 & 5 & 2.53 & 0.3 & 9 & 90 & 93.606 & 0.115 \\
\hline Pd(OAC)2 & KHCO3 & Dioxane & 24 & 6 & 5 & 2.54 & 0.2 & 12 & 90 & 89.055 & 3.112 \\
\hline $\mathrm{Pd}(O A C) 2$ & KHCO3 & Dloxane & 24 & 6 & 5 & 2.90 & 0.2 & 9 & 90 & 93.233 & 0.501 \\
\hline $\mathrm{Pd}(\mathrm{OAC}) 2$ & KHCO3 & Dloxane & 24 & 10 & 5 & 2.56 & 0.2 & 9 & 90 & 92.734 & 0 \\
\hline $\mathrm{Pd}\left(\mathrm{OAC}_{\mathrm{C}}\right) 2$ & KHCO3 & Dloxane & 24 & 6 & 7 & 2.50 & 0.2 & 9 & 90 & 91.183 & 0.17 \\
\hline $\mathrm{Pd}(\mathrm{OAC}) 2$ & KHCO3 & Dloxane & 24 & 2 & 3 & 2.00 & 0.1 & 6 & 100 & 23.748 & 69.384 \\
\hline $\mathrm{Pd}\left(\mathrm{OAC}_{\mathrm{C}}\right) 2$ & KHCO3 & Dloxane & 24 & 2 & 3 & 1.87 & 0.3 & 12 & 100 & 22.864 & 72.244 \\
\hline$P d(O A C) 2$ & KHCO3 & Dloxane & 24 & 2 & 3 & 2.96 & 0.3 & 6 & 100 & 22.481 & 72.511 \\
\hline$P d(O A C) 2$ & KHCO3 & Dloxane & 24 & 2 & 3 & 3.00 & 0.1 & 12 & 100 & 22.411 & 73.686 \\
\hline $\mathrm{Pd}(\mathrm{OAC}) 2$ & KHCO3 & Dloxane & 24 & 10 & 3 & 2.00 & 0.3 & 6 & 100 & 92.974 & 0.056 \\
\hline $\mathrm{Pd}(\mathrm{OAC}) 2$ & KHCO3 & Dloxane & 24 & 10 & 3 & 2.01 & 0.1 & 12 & 100 & 90.389 & 0.081 \\
\hline $\mathrm{Pd}(\mathrm{OAC}) 2$ & KHCO3 & Dloxane & 24 & 10 & 3 & 2.93 & 0.1 & 6 & 100 & 91.548 & 0 \\
\hline $\mathrm{Pd}(\mathrm{OAC}) 2$ & KHCO3 & Dloxane & 24 & 10 & 3 & 2.95 & 0.3 & 12 & 100 & 90.882 & 0 \\
\hline $\mathrm{Pd}\left(\mathrm{OAC}_{\mathrm{C}}\right) 2$ & KHCO3 & Dloxane & 24 & 6 & 5 & 2.42 & 0.2 & 9 & 100 & 93.65 & 0 \\
\hline $\mathrm{Pd}(\mathrm{OAC}) 2$ & $\mathrm{KHCO} 3$ & Dloxane & 24 & 2 & 7 & 2.02 & 0.3 & 6 & 100 & 84.762 & 0 \\
\hline $\mathrm{Pd}(\mathrm{OAC}) 2$ & KHCO3 & Dloxane & 24 & 2 & 7 & 2.00 & 0.1 & 12 & 100 & 30.603 & 65.995 \\
\hline $\mathrm{Pd}(\mathrm{OAC}) 2$ & KHCO3 & Dloxane & 24 & 2 & 7 & 2.94 & 0.1 & 6 & 100 & 32.953 & 60.939 \\
\hline Pd(OAC)2 & $\mathrm{KHCO}$ & Dloxane & 24 & 2 & 7 & 3.00 & 0.3 & 12 & 100 & 26.315 & 68.967 \\
\hline $\mathrm{Pd}(\mathrm{OAC}) 2$ & KHCO3 & Dloxane & 24 & 10 & 7 & 1.93 & 0.1 & 6 & 100 & 90.365 & 0 \\
\hline $\mathrm{Pd}(\mathrm{OAC}) 2$ & KHCO3 & Dloxane & 24 & 10 & 7 & 2.04 & 0.3 & 12 & 100 & 89.614 & 0 \\
\hline $\mathrm{Pd}(O A C) 2$ & KHCO3 & Dloxane & 24 & 10 & 7 & 2.99 & 0.3 & 6 & 100 & 87.992 & 1.09 \\
\hline$P d(O A C) 2$ & $\mathrm{KHCO}$ & Dloxane & 24 & 10 & 7 & 3.07 & 0.1 & 12 & 100 & 87.943 & 0 \\
\hline
\end{tabular}


Screen $\# 4$

\begin{tabular}{|c|c|c|c|c|c|c|c|c|c|c|c|}
\hline & का & Pd & $\mathrm{ACOH}$ & & Addiltwe & & & remp & & Pdit: & sM \\
\hline umol & equilv & 5 mol \% & equlv & Equilv & 1.0 equlv & Name & rol & ${ }^{\circ} \mathrm{C}$ & n & $A P$ & $A P$ \\
\hline 10 & 10.0 & $P \square(O A C) 2$ & 0.2 & 3.00 & None & Anlsole & 1.50 & 113 & 24.0 & $10.11 \%$ & $87.46 \%$ \\
\hline 10 & 10.0 & $\mathrm{Pd}(\mathrm{OAC}) 2$ & 0.2 & 3.00 & $\mathbf{K l}$ & Anlsole & 1.50 & 113 & 24.0 & $10.04 \%$ & $87.17 \%$ \\
\hline 10 & 10.0 & $P d O A C / 2$ & 0.2 & 3.00 & KPF6 & Anlsole & 1.50 & 113 & 24.0 & $11.75 \%$ & 0.85425 \\
\hline 10 & 10.0 & $P d O A C) 2$ & 0 & 3.00 & KOTI & Anlsole & 1.50 & 113 & 24.0 & $13.87 \%$ & 0.83144 \\
\hline 10 & 10.0 & PdOAC)2 & 0.2 & 3.00 & KOTT & Anlsole & 1.50 & 113 & 24.0 & $12.61 \%$ & 0.84418 \\
\hline 10 & 10.0 & $\mathrm{Pd} / \mathrm{OAC} / 2$ & 0.2 & 3.00 & TBAOTI & Anlsole & 1.50 & 113 & 24.0 & 0 & $89.80 \%$ \\
\hline 10 & 10.0 & $\mathrm{Pd}(\mathrm{OAC}) 2$ & 0.2 & 3.00 & None & Anlsole & 1.50 & 113 & 24.0 & 0 & $75.99 \%$ \\
\hline 10 & 10.0 & $\mathrm{Pd}(\mathrm{OAC}) 2$ & 0.2 & 3.00 & KI & Anlsole & 1.50 & 113 & 24.0 & $19.50 \%$ & $77.23 \%$ \\
\hline 10 & 10.0 & $P d(O A c) / 2$ & 0.2 & 3.00 & KPF6 & Anlsole & 1.50 & 113 & 24.0 & $22.16 \%$ & $74.66 \%$ \\
\hline 10 & 10.0 & $P d(O A C) 2$ & 0 & 3.00 & KOTT & Anlsole & 1.50 & 113 & 24.0 & $23.92 \%$ & $72.96 \%$ \\
\hline $\begin{array}{l}10 \\
10\end{array}$ & $\begin{array}{l}10.0 \\
10.0\end{array}$ & $\begin{array}{l}\mathrm{Pd}(O A C) / 2 \\
\mathrm{Pd}(\mathrm{OAC} / 2\end{array}$ & $\begin{array}{l}0.2 \\
0.2\end{array}$ & $\begin{array}{l}3.00 \\
3.00\end{array}$ & $\begin{array}{c}\text { KOTT } \\
\text { TBAOTI }\end{array}$ & $\begin{array}{l}\text { Anlsole } \\
\text { Anlsole }\end{array}$ & $\begin{array}{l}1.50 \\
1.50\end{array}$ & $\begin{array}{l}113 \\
113\end{array}$ & $\begin{array}{l}24.0 \\
24.0\end{array}$ & $\begin{array}{c}23.92 \% \\
9.68 \%\end{array}$ & $\begin{array}{l}73.22 \% \\
87.01 \%\end{array}$ \\
\hline 10 & $10.0^{\circ}$ & Pव:OAC)/2 & 0.2 & $3.00^{\circ}$ & None & Anाsole & 1.50 & 113 & 24.0 & $29.19 \%$ & $67.21 \%$ \\
\hline 10 & 10.0 & $\mathrm{Pd}(\mathrm{OAC}) 2$ & 0.2 & 3.00 & $\mathbf{K a}$ & Anlsole & 1.50 & 113 & 24.0 & $27.81 \%$ & $68.54 \%$ \\
\hline 10 & 10.0 & $P d O A C) 2$ & 0.2 & 3.00 & KPF6 & Anlsole & 1.50 & 113 & 24.0 & $29.33 \%$ & $66.94 \%$ \\
\hline 10 & 10.0 & $P d O A C) 2$ & 0 & 3.00 & KOTT & Anlsole & 1.50 & 113 & 24.0 & $26.66 \%$ & $69.33 \%$ \\
\hline 10 & 10.0 & $P d(O A C) 2$ & 0.2 & 3.00 & KOTI & Anlisole & 1.50 & 113 & 24.0 & $27.33 \%$ & $69.20 \%$ \\
\hline 10 & 10.0 & $P d(O A C) 2$ & 0.2 & 3.00 & TBAOTI & Anlsole & 1.50 & 113 & 24.0 & $11.55 \%$ & $85.41 \%$ \\
\hline 10 & 10.0 & $\mathrm{Pd} / \mathrm{OAC} / 2$ & 0.2 & 3.00 & None & Anlsole & 1.50 & 113 & 24.0 & $28.45 \%$ & $66.98 \%$ \\
\hline 10 & 10.0 & $P d O A C) 2$ & 0.2 & 3.00 & KI & Anlsole & 1.50 & 113 & 24.0 & $32.16 \%$ & $63.64 \%$ \\
\hline 10 & 10.0 & $P d(O A c) 2$ & 0.2 & 3.00 & KPF6 & Anlsole & 1.50 & 113 & 24.0 & $31.37 \%$ & $64.09 \%$ \\
\hline 10 & 10.0 & $\mathrm{Pd}(\mathrm{OAC}) 2$ & 0 & 3.00 & KOTI & Anlsole & 1.50 & 113 & 24.0 & $39.50 \%$ & $56.59 \%$ \\
\hline 10 & 10.0 & $\begin{array}{l}\mathrm{Pd}(O A C) / 2 \\
\mathrm{Pd}(O A C / 2\end{array}$ & 0.2 & $\begin{array}{l}3.00 \\
3.00\end{array}$ & $\begin{array}{c}\text { KOTT } \\
\text { TBAOTI }\end{array}$ & $\begin{array}{l}\text { Anlsole } \\
\text { Anisole }\end{array}$ & $\begin{array}{l}1.50 \\
1.50\end{array}$ & $\begin{array}{l}113 \\
113\end{array}$ & 24.0 & $62.41 \%$ & $26.23 \%$ \\
\hline 10 & $70.0^{\circ}$ & $P \sigma(O A C) / 2$ & 0.2 & $3.00^{\circ}$ & None & Anlsole & $1.50^{\circ}$ & 113 & 24.0 & $61.32 \%$ & $28.55 \%$ \\
\hline 10 & 10.0 & $\mathrm{Pd} / \mathrm{OAC}) 2$ & 0.2 & 3.00 & KI & Anlsole & 1.50 & 113 & 24.0 & $62.61 \%$ & $27.19 \%$ \\
\hline 10 & 10.0 & $P d(O A C) 2$ & 0.2 & 3.00 & KPF6 & Anlsole & 1.50 & 113 & 24.0 & $64.05 \%$ & $25.88 \%$ \\
\hline 10 & 10.0 & $P d O A C) 2$ & 0 & 3.00 & KOTI & Anlsole & 1.50 & 113 & 24.0 & $60.25 \%$ & $27.31 \%$ \\
\hline 10 & 10.0 & $P d(O A C) 2$ & 0.2 & 3.00 & KOTT & Anlsole & 1.50 & 113 & 24.0 & $63.12 \%$ & $25.15 \%$ \\
\hline 10 & 10.0 & $P d(O A C) 2$ & 0.2 & 3.00 & TBAOTI & Anlsole & 1.50 & 113 & 24.0 & $19.51 \%$ & $70.80 \%$ \\
\hline 10 & 10.0 & $\mathrm{Pd} / O A C) 2$ & 0.2 & 3.00 & None & Anlsole & 1.50 & 113 & 24.0 & $71.10 \%$ & $19.16 \%$ \\
\hline 10 & 10.0 & $\mathrm{Pd} / O A C) 2$ & 0.2 & 3.00 & $\mathbf{K l}$ & Anlsole & 1.50 & 113 & 24.0 & $69.11 \%$ & $20.27 \%$ \\
\hline 10 & 10.0 & $\mathrm{Pd}(\mathrm{OAC}) 2$ & 0.2 & 3.00 & KPF6 & Anlsole & 1.50 & 113 & 24.0 & $67.95 \%$ & $20.90 \%$ \\
\hline 10 & 10.0 & $P d(O A c) / 2$ & 0 & 3.00 & KOTI & Anlsole & 1.50 & 113 & 24.0 & $70.32 \%$ & $18.10 \%$ \\
\hline 10 & 10.0 & $P d(O A c) 2$ & 0.2 & 3.00 & KOTT & Anlsole & 1.50 & 113 & 24.0 & $70.23 \%$ & $17.75 \%$ \\
\hline 10 & 10.0 & $\mathrm{Pd} O A C / 2$ & 0.2 & 3.00 & TBAOTI & Anlisole & 1.50 & 113 & 24.0 & $20.02 \%$ & $69.36 \%$ \\
\hline
\end{tabular}


3creen $* 5$

\begin{tabular}{|c|c|c|c|c|c|c|c|c|c|c|c|c|c|}
\hline \multirow{2}{*}{$\begin{array}{c}8 \mathrm{gm} \\
\mathrm{ymol}\end{array}$} & \multirow{2}{*}{$\begin{array}{l}\text { Phl } \\
\text { oguly }\end{array}$} & \multirow{2}{*}{$\begin{array}{c}\text { Pd } \\
6 \mathrm{~mol} \mathrm{\%}\end{array}$} & \multirow{2}{*}{$\begin{array}{l}\text { Additivo } \\
5 \mathrm{~mol} \%\end{array}$} & \multirow{2}{*}{$\begin{array}{l}\text { Additivo } \\
\text { Equiv }\end{array}$} & \multicolumn{2}{|c|}{ Bace } & \multirow{2}{*}{$\begin{array}{l}\text { Additivo } \\
10 \mathrm{~mol} \%\end{array}$} & \multirow[b]{2}{*}{ Name } & \multicolumn{3}{|c|}{ Tomp } & \multirow{2}{*}{$\begin{array}{l}\text { Pdt: } \\
\text { AP }\end{array}$} & \multirow{2}{*}{${ }_{A P}$} \\
\hline & & & & & Name & Equlv & & & voll & ${ }^{\circ} \mathrm{C}$ & h & & \\
\hline 10 & 100 & Pd(OAC)2 & DIMPU & 2 & $\mathrm{KHCO} 3$ & 3.00 & ACOH & Antsole & 4.00 & 110 & 24.0 & $16.19 \%$ & $74.15 \%$ \\
\hline 10 & 100 & $P d(O A c) 2$ & DMPU & 4 & $\mathrm{KHCO}_{3}$ & 3.00 & $\mathrm{ACOH}$ & Anleole & 4.00 & 110 & 24.0 & $16.51 \%$ & $61.79 \%$ \\
\hline 10 & 100 & Pdionc $)_{2}$ & DMPU & 6 & $\mathrm{KHCO}_{3}$ & 3.00 & $\mathrm{ACOH}$ & Anbole & 4.00 & 110 & 24.0 & $7.08 \%$ & 0.73252 \\
\hline 10 & 100 & $P d(O A c) / 2$ & & & $\mathrm{KHCO}_{3}$ & 3.00 & $\mathrm{ACOH}$ & Dloxane & 4.00 & 110 & 24.0 & $56.61 \%$ & 0.40062 \\
\hline 10 & 100 & $\operatorname{Pd}(\mathrm{O} A \mathrm{Ac}) 2$ & & & TEA & 3.00 & $\mathrm{ACOH}$ & Dloxane & 4.00 & 110 & 24.0 & $3.02 \%$ & 0.7397 \\
\hline 10 & 100 & PdiOAc) 2 & & & DABCO & 3.00 & $\mathrm{ACOH}$ & Dloxane & 4.00 & 110 & 24.0 & 0 & $51.81 \%$ \\
\hline 10 & 100 & PdiOAc)/2 & & & DIPEA & 3.00 & $\mathrm{ACOH}$ & Dloxane & 4.00 & 110 & 24.0 & 0 & $84.83 \%$ \\
\hline 10 & 100 & $P d(O A c) 2$ & & & ктомв & 3.00 & $\mathrm{ACOH}$ & Dloxane & 4.00 & 110 & 24.0 & $221 \%$ & $75.42 \%$ \\
\hline 10 & 100 & $\mathrm{Pd}(\mathrm{OAC}) / 2$ & & & 2,6 -lutidine & 3.00 & $\mathrm{ACOH}$ & Dloxane & 4.00 & 110 & 24.0 & $8.03 \%$ & $86.65 \%$ \\
\hline 10 & 100 & Pdilonc $) 2$ & & & DMNP & 3.00 & $\mathrm{ACOH}$ & Dloxane & 4.00 & 110 & 24.0 & $1.91 \%$ & $94.60 \%$ \\
\hline $\begin{array}{l}10 \\
10\end{array}$ & $\begin{array}{l}100 \\
100\end{array}$ & $\begin{array}{l}\text { Pd(OAc) } 12 \\
\operatorname{Pd}\left(O_{A C}\right) / 2\end{array}$ & & & $\begin{array}{c}\text { Deu } \\
\text { CyzaNMe }\end{array}$ & $\begin{array}{r}3.00 \\
3.00\end{array}$ & $\begin{array}{l}\mathrm{ACOH} \\
\mathrm{ACOH}\end{array}$ & $\begin{array}{l}\text { Dloxane } \\
\text { Dloxane }\end{array}$ & $\begin{array}{l}4.00 \\
4.00\end{array}$ & $\begin{array}{l}110 \\
110\end{array}$ & $\begin{array}{l}24.0 \\
24.0\end{array}$ & $\begin{array}{l}0.41 \% \\
2.12 \%\end{array}$ & $\begin{array}{l}96.40 \% \\
72.98 \%\end{array}$ \\
\hline 70 & 1000 & pratore $)^{2}$ & DIMAC & 2 & RHCOOS & 3.000 & ACOAF & Anare & $4.00^{\circ}$ & $170^{\circ}$ & 2400 & $51.64 \%$ & $26.97 \%$ \\
\hline 10 & 100 & Pdionc $) 2$ & DMAC & 4 & $\mathrm{KHCO}_{3}$ & 3.00 & $\mathrm{ACOH}$ & Antsole & 4.00 & 110 & 24.0 & $30.03 \%$ & $46.81 \%$ \\
\hline 10 & 100 & $\operatorname{Pd}\left(\mathrm{OAAC}_{\mathrm{A}}\right) 2$ & DMAC & 6 & $\mathrm{KHCO}_{3}$ & 3.00 & $\mathrm{ACOH}$ & Anleole & 4.00 & 110 & 24.0 & $19.89 \%$ & $58.50 \%$ \\
\hline 10 & 100 & PdiOAc) 2 & & & $\mathrm{KHCO} 3$ & 3.00 & & Anleole & 4.00 & 110 & 24.0 & $52.25 \%$ & $44.39 \%$ \\
\hline 10 & 100 & $P d(O A c) 2$ & & & TEA & 3.00 & & Anbole & 4.00 & 110 & 24.0 & $5.41 \%$ & $65.96 \%$ \\
\hline 10 & 10.0 & $P d(O A c) 2$ & & & DABCO & 3.00 & & Anbole & 4.00 & 110 & 24.0 & $3.90 \%$ & $49.42 \%$ \\
\hline 10 & 100 & $P d(O A c) 2$ & & & DIPEA & 3.00 & & Anlsole & 4.00 & 110 & 24.0 & $2.85 \%$ & $85.29 \%$ \\
\hline 10 & 100 & Pdionc $) 2$ & & & ктомв & 3.00 & & Anlsole & 4.00 & 110 & 24.0 & $17.49 \%$ & $75.42 \%$ \\
\hline 10 & 100 & PdiOAc) 2 & & & 2,6-utudine & 3.00 & & Anlsole & 4.00 & 110 & 24.0 & $7.81 \%$ & $86.34 \%$ \\
\hline 10 & 10.0 & $P d(O A c) / 2$ & & & DMNP & 3.00 & & Anbole & 4.00 & 110 & 24.0 & $1.71 \%$ & $93.55 \%$ \\
\hline $\begin{array}{l}10 \\
10\end{array}$ & $\begin{array}{l}10.0 \\
10.0\end{array}$ & $\begin{array}{l}\text { Pd(OAc) } 2 \\
\text { Pd(OAC) } 2\end{array}$ & & & $\begin{array}{l}\text { DeU } \\
\text { Cyz2NMe }\end{array}$ & $\begin{array}{l}3.00 \\
3.00\end{array}$ & & $\begin{array}{l}\text { Anbole } \\
\text { Antsole }\end{array}$ & $\begin{array}{l}4.00 \\
4.00\end{array}$ & $\begin{array}{l}110 \\
110\end{array}$ & $\begin{array}{l}24.0 \\
24.0\end{array}$ & $\begin{array}{l}0.52 \% \\
2.09 \%\end{array}$ & $\begin{array}{l}92.65 \% \\
74.78 \%\end{array}$ \\
\hline 10 & 100 & PdiOAc/2 & NMP & 2 & $\mathrm{KHCO} 3$ & 3.00 & ACOH & Anisole & 4.00 & 110 & 24.0 & $66.37 \%$ & $16.01 \%$ \\
\hline 10 & 100 & Pdilonc) 2 & NMP & 4 & $\mathrm{KHCO} 3$ & 3.00 & $\mathrm{ACOH}$ & Anbole & 4.00 & 110 & 24.0 & $26.39 \%$ & $50.83 \%$ \\
\hline 10 & 100 & Pdionc $) 2$ & NMP & 6 & $\mathrm{KHCO}_{3}$ & 3.00 & $\mathrm{ACOH}$ & Anbole & 4.00 & 110 & 24.0 & $15.87 \%$ & $62.71 \%$ \\
\hline 10 & 100 & $P d(O A c) / 2$ & & & $\mathrm{KHCO} 3$ & 3.00 & $\mathrm{ACOH}$ & Dloxane & 4.00 & 110 & 24.0 & $58.48 \%$ & $38.24 \%$ \\
\hline 10 & 100 & $P d(O A c) 2$ & & & TEA & 3.00 & $\mathrm{ACOH}$ & Dloxane & 4.00 & 110 & 24.0 & $3.30 \%$ & $84.84 \%$ \\
\hline 10 & 100 & Pdionc $) 2$ & & & DABCO & 3.00 & $\mathrm{ACOH}$ & Dloxane & 4.00 & 110 & 24.0 & $3.34 \%$ & $59.22 \%$ \\
\hline 10 & 10.0 & $P d(O A c) 2$ & & & DIPEA & 3.00 & $\mathrm{AcOH}$ & Dloxane & 4.00 & 110 & 24.0 & $2.98 \%$ & $79.45 \%$ \\
\hline 10 & 100 & $P d(O A C) 2$ & & & КтомВ & 3.00 & $\mathrm{ACOH}$ & Dloxane & 4.00 & 110 & 24.0 & $38.85 \%$ & $47.64 \%$ \\
\hline 10 & 100 & $P d(O A C) / 2$ & & & 2,6 -lutidine & 3.00 & $\mathrm{ACOH}$ & Dloxane & 4.00 & 110 & 24.0 & $17.05 \%$ & $75.25 \%$ \\
\hline 10 & 100 & $\operatorname{Pd}\left(\mathrm{OANC}_{\mathrm{A}}\right) 2$ & & & DMAP & 3.00 & $\mathrm{ACOH}$ & Dloxane & 4.00 & 110 & 24.0 & $1.16 \%$ & $88.72 \%$ \\
\hline 10 & 100 & Pd $(O A C) 2$ & & & DEU & 3.00 & $\mathrm{ACOH}$ & Dloxane & 4.00 & 110 & 24.0 & $0.59 \%$ & $92.80 \%$ \\
\hline 10 & 100 & $\operatorname{Pd}\left(\mathrm{OANC}^{\prime}\right) 2$ & & & CyzanMe & 3.00 & $\mathrm{ACOH}$ & Dloxane & 4.00 & 110 & 24.0 & $2.13 \%$ & $69.80 \%$ \\
\hline 10 & 100 & Pd(OAC)/2 & DMBO & 2 & $\mathrm{KHCO} 3$ & 3.00 & ACOH & Antrole & 4.00 & 110 & 24.0 & $40.69 \%$ & $37.43 \%$ \\
\hline 10 & 100 & PdiOAc) 2 & DMBO & 4 & $\mathrm{KHCO}_{3}$ & 3.00 & $\mathrm{ACOH}$ & Anbole & 4.00 & 110 & 24.0 & $17.35 \%$ & $59.49 \%$ \\
\hline 10 & 100 & $P d(O A c) 2$ & DMBO & 6 & $\mathrm{KHCO} 3$ & 3.00 & $\mathrm{AcOH}$ & Anbole & 4.00 & 110 & 24.0 & $11.38 \%$ & $78.93 \%$ \\
\hline 10 & 100 & $P d(O A c) 2$ & & & $\mathrm{KHCO} 3$ & 3.00 & & Antsole & 4.00 & 110 & 24.0 & $55.52 \%$ & $40.54 \%$ \\
\hline 10 & 10.0 & Pd $(O A C) 2$ & & & TEA & 3.00 & & Anbole & 4.00 & 110 & 24.0 & $2.80 \%$ & $70.44 \%$ \\
\hline 10 & 10.0 & Pdiloncl2 2 & & & DABCO & 3.00 & & Anbole & 4.00 & 110 & 24.0 & $3.27 \%$ & $49.37 \%$ \\
\hline 10 & 100 & Pd $(O A C) 2$ & & & DIPEA & 3.00 & & Anbole & 4.00 & 110 & 24.0 & $3.46 \%$ & $75.02 \%$ \\
\hline 10 & 100 & $P d(O A c) / 2$ & & & ктомв & 3.00 & & Anbole & 4.00 & 110 & 24.0 & $18.29 \%$ & $58.85 \%$ \\
\hline 10 & 100 & Pdionc $) 2$ & & & 2,6 -lutidine & 3.00 & & Anbole & 4.00 & 110 & 24.0 & $11.18 \%$ & $56.39 \%$ \\
\hline 10 & 10.0 & PdiOAc) 2 & & & DMAP & 3.00 & & Anbole & 4.00 & 110 & 24.0 & $0.79 \%$ & $81.30 \%$ \\
\hline $\begin{array}{l}10 \\
10\end{array}$ & 100 & PdiOAc)/2 & & & DBU & 3.00 & & Anbole & $\begin{array}{l}4.00 \\
4.00\end{array}$ & $\begin{array}{l}110 \\
110\end{array}$ & 24.0 & $0.41 \%$ & $79.72 \%$ \\
\hline & & $\mathrm{Pd}(\mathrm{OA} C) 2$ & & & & 3.00 & & & 4.00 & 110 & 24.0 & $1.72 \%$ & $56.96 \%$ \\
\hline
\end{tabular}




\begin{tabular}{|c|c|c|c|c|c|c|c|c|c|c|c|c|c|}
\hline \multirow{2}{*}{$\begin{array}{l}\text { an } \\
\text { umol }\end{array}$} & \multirow{2}{*}{$\begin{array}{l}\text { Phit } \\
\text { oquiv }\end{array}$} & \multirow{2}{*}{$6 \mathrm{~mol} \%$} & \multirow{2}{*}{$\begin{array}{l}\text { Afdalitivo } \\
6 \mathrm{~mol} 4 \%\end{array}$} & \multirow{2}{*}{$\begin{array}{l}\text { Adaritive } \\
\text { Equlv }\end{array}$} & \multicolumn{2}{|c|}{ вबटе } & \multirow{2}{*}{$\begin{array}{l}\text { Addalitive } \\
10 \mathrm{~mol} \%\end{array}$} & \multirow[b]{2}{*}{ Name } & \multicolumn{3}{|c|}{ vemp } & \multirow{2}{*}{$\begin{array}{l}\text { Pai: } \\
\text { AP }\end{array}$} & \multirow{2}{*}{ AP } \\
\hline & & & & & Name & Equlv & & & wol & ${ }^{\circ} \mathrm{C}$ & h & & \\
\hline 10 & 10.0 & PdOAC]2 & DMPU & 2 & $\mathrm{KHCO} 3$ & 300 & $\mathrm{ACOH}$ & Dioxane & 4.00 & 110 & 240 & $17.34 \%$ & $68.71 \%$ \\
\hline 10 & 10.0 & PdIOAC; 2 & DMPU & 4 & КHCO3 & 300 & $\mathrm{ACOH}$ & Dloxane & 4.00 & 110 & 240 & $15.61 \%$ & $62.25 \%$ \\
\hline 10 & 10.0 & Pd $(O A C) \mid 2$ & DMPU & 6 & $\mathrm{KHCO}$ & 300 & $\mathrm{ACOH}$ & Dloxane & 4.00 & 110 & 240 & $8.42 \%$ & $69.75 \%$ \\
\hline 10 & 10.0 & PdIOAc) ${ }^{2}$ & DMPU & 2 & $\mathrm{KHCO}$ & 300 & $\mathrm{ACOH}$ & Anlsole & 4.00 & 110 & 240 & $51.07 \%$ & $31.17 \%$ \\
\hline 10 & 10.0 & PdiOAc) 12 & DMPU & 2 & TEA & 300 & $\mathrm{ACOH}$ & Anlsole & 4.00 & 110 & 240 & $2.73 \%$ & $59.15 \%$ \\
\hline 10 & 10.0 & PdIOAC) 2 & DMPU & 2 & DABCO & 300 & $\mathrm{ACOH}$ & Anisole & 4.00 & 110 & 240 & $2.11 \%$ & $37.19 \%$ \\
\hline 10 & 10.0 & Pd(OPN) & DMPU & 2 & DIPEA & 300 & $\mathrm{ACOH}$ & Anlsole & 4.00 & 110 & 240 & $1.96 \%$ & $63.30 \%$ \\
\hline 10 & 10.0 & Pd(OPI) 2 & DMPU & 2 & ктомя & 300 & $\mathrm{ACOH}$ & Anisole & 4.00 & 110 & 240 & $4.69 \%$ & $56.36 \%$ \\
\hline 10 & 10.0 & Pd(OPIN]2 & DMPU & 2 & 2,6-lubdine & 300 & $\mathrm{ACOH}$ & Arisole & 4.00 & 110 & 240 & $4.13 \%$ & $62.55 \%$ \\
\hline 10 & 10.0 & Pd $(O A C) \mid 2$ & DMPU & 2 & DMNP & 300 & $\mathrm{ACOH}$ & Arisole & 4.00 & 110 & 240 & $1.53 \%$ & $79.81 \%$ \\
\hline $\begin{array}{l}10 \\
10\end{array}$ & $\begin{array}{l}10.0 \\
10.0\end{array}$ & $\begin{array}{l}\text { PdIOAC) } 2 \\
\text { PdIOAC) }\end{array}$ & $\begin{array}{l}\text { DMPU } \\
\text { DMPU }\end{array}$ & 2 & $\begin{array}{c}\text { DEU } \\
\text { Cy2NMe }\end{array}$ & $\begin{array}{l}300 \\
300\end{array}$ & $\begin{array}{l}\mathrm{ACOH} \\
\mathrm{ACOH}\end{array}$ & $\begin{array}{l}\text { Arisole } \\
\text { Arisole }\end{array}$ & $\begin{array}{l}4.00 \\
4.00\end{array}$ & $\begin{array}{l}110 \\
110\end{array}$ & 240 & $\begin{array}{l}0.57 \% \\
1.97 \%\end{array}$ & $\begin{array}{l}82.75 \% \\
63.47 \%\end{array}$ \\
\hline Fiv & Fots & Fб(O) & DIMGE & 2 & TheOOS & 3000 & ACOOH & bioxane & 2800 & Filo & 240 & $41.92 \%$ & $35.73 \%$ \\
\hline 10 & 10.0 & PdiOAc) 2 & DMac & 4 & $\mathrm{KHCO}$ & 300 & $\mathrm{ACOH}$ & Dloxane & 4.00 & 110 & 240 & $23.57 \%$ & $51.58 \%$ \\
\hline 10 & 10.0 & PdiOAC) 2 & DMac & 6 & КHCO3 & 300 & $\mathrm{ACOH}$ & Dloxane & 4.00 & 110 & 240 & $16.89 \%$ & $59.33 \%$ \\
\hline 10 & 10.0 & PdIOAC) 2 & DMac & 2 & KHCO3 & 300 & $\mathrm{ACOH}$ & Anisole & 4.00 & 110 & 240 & $32.96 \%$ & $35.50 \%$ \\
\hline 10 & 10.0 & PdIOAC) 2 & DMAc & 2 & TEA & 300 & $\mathrm{ACOH}$ & Anisole & 4.00 & 110 & 240 & $2.66 \%$ & $58.08 \%$ \\
\hline 10 & 10.0 & PdIOAC) 2 & DMac & 2 & DABCO & 300 & $\mathrm{ACOH}$ & Anlsole & 4.00 & 110 & 240 & $2.82 \%$ & $32.06 \%$ \\
\hline 10 & 10.0 & PdiOAc) 12 & DMac & 2 & DIPEA & 300 & $\mathrm{ACOH}$ & Anisole & 4.00 & 110 & 240 & $2.62 \%$ & $63.06 \%$ \\
\hline 10 & 10.0 & PdIOAc) 12 & DMAc & 2 & Ктомв & 3.00 & $\mathrm{ACOH}$ & Anisole & 4.00 & 110 & 240 & $6.20 \%$ & $56.69 \%$ \\
\hline 10 & 10.0 & PdIOAC)/2 & DMac & 2 & 2, 6-lubdine & 300 & $\mathrm{ACOH}$ & Arisole & 4.00 & 110 & 240 & $5.06 \%$ & $64.31 \%$ \\
\hline 10 & 10.0 & PdiOAc) $\mid 2$ & DMare & 2 & DMAP & 3.00 & $\mathrm{ACOH}$ & Anlsole & 4.00 & 110 & 240 & $1.22 \%$ & $70.02 \%$ \\
\hline 10 & 10.0 & PdIOAc) 2 & DMAc & 2 & DEU & 3.00 & $\mathrm{ACOH}$ & Anlsole & 4.00 & 110 & 240 & $0.00 \%$ & $68.41 \%$ \\
\hline 10 & 10.0 & $P d(O A C) / 2$ & DMAc & 2 & CyzNMe & 3.00 & $\mathrm{ACOH}$ & Anisole & 4.00 & 110 & 240 & $2.40 \%$ & $63.00 \%$ \\
\hline Fiv & Fotes & 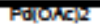 & TNOF- & 2000 & ketcos & 3000 & ACOH & bioxane & 4000 & Triv & 240 & $38.94 \%$ & $52.92 \%$ \\
\hline 10 & 10.0 & Pd $(O A \mid C) 2$ & NMP & 400 & КHCO3 & 300 & $\mathrm{ACOH}$ & Dloxane & 4.00 & 110 & 240 & $23.09 \%$ & $67.58 \%$ \\
\hline 10 & 10.0 & PdIOAC) 2 & NQP & 600 & KHCO3 & 300 & $\mathrm{ACOH}$ & Dloxane & 4.00 & 110 & 240 & $17.37 \%$ & $56.46 \%$ \\
\hline 10 & 10.0 & PdiOAc) 2 & NMP & 200 & КHCO3 & 300 & $\mathrm{ACOH}$ & Anleole & 4.00 & 110 & 240 & $34.90 \%$ & $41.39 \%$ \\
\hline 10 & 10.0 & PdIOAc]2 2 & NMP & 200 & TEA & 300 & $\mathrm{ACOH}$ & Anlsole & 4.00 & 110 & 240 & $2.42 \%$ & $58.86 \%$ \\
\hline 10 & 10.0 & PdIOAC)/2 & NuP & 200 & DABCO & 300 & $\mathrm{ACOH}$ & Anisole & 4.00 & 110 & 240 & $2.28 \%$ & $36.53 \%$ \\
\hline 10 & 10.0 & PdIOAC) 2 & NMP & 200 & DIPEA & 300 & $\mathrm{ACOH}$ & Anisole & 4.00 & 110 & 240 & $2.32 \%$ & $63.18 \%$ \\
\hline 10 & 10.0 & PdIOAC;2 2 & NuP & 200 & ктомs & 300 & $\mathrm{ACOH}$ & Anisole & 4.00 & 110 & 240 & $9.38 \%$ & $56.51 \%$ \\
\hline 10 & 10.0 & PdIOAc $)_{2}$ & NMP & 200 & 2,6-ludidine & 300 & $\mathrm{ACOH}$ & Arlsole & 4.00 & 110 & 240 & $0.00 \%$ & $64.75 \%$ \\
\hline 10 & 10.0 & PdIOAC) 2 & NMP & 200 & DMNP & 300 & $\mathrm{ACOH}$ & Anisole & 4.00 & 110 & 240 & $1.20 \%$ & $68.56 \%$ \\
\hline $\begin{array}{l}10 \\
10\end{array}$ & $\begin{array}{l}10.0 \\
10.0\end{array}$ & $\begin{array}{l}\text { PdIOAC], } \\
\text { PdIOAC: }\end{array}$ & $\begin{array}{l}\text { NQPP } \\
\text { NMP }\end{array}$ & $\begin{array}{l}200 \\
200\end{array}$ & $\begin{array}{l}\text { DEU } \\
\text { CORNME }\end{array}$ & 3.00 & $\mathrm{ACOH}$ & Arisole & 4.00 & 110 & 240 & $0.52 \%$ & $68.88 \%$ \\
\hline fin & foll: & Podonclat & Dowero & 200 & 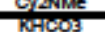 & 3000 & $\mathrm{ACOAH}$ & Nribore & 2.00 & EElot & 240 & . & $56.56 \%$ \\
\hline 10 & . & FofoAc/2 & DMESO & 200 & $\mathrm{KHCOOS}$ & 300 & ACOA & Dioxane & 4.00 & 110 & 240 & $0.00 \%$ & $49.65 \%$ \\
\hline & we. & $P d(O A C) 2$ & DMSO & 400 & $\mathrm{KHCO}$ & 300 & $\mathrm{ACOH}$ & Dioxane & 4.00 & 110 & 240 & $0.00 \%$ & $61.78 \%$ \\
\hline id & 100 & Pd $(O A C), 2$ & DMSO & 600 & $\mathrm{KHCO} 3$ & 300 & $\mathrm{ACOH}$ & Dloxane & 4.00 & 110 & 240 & $12.71 \%$ & $77.33 \%$ \\
\hline 10 & 10.0 & PdIOAC $)^{2}$ & DMSO & 200 & $\mathrm{KHCO} 3$ & 300 & $\mathrm{AcOH}$ & Anlisole & 4.00 & 110 & 240 & $50.35 \%$ & $38.12 \%$ \\
\hline 10 & 10.0 & PdiOAc) 2 & DMSO & 200 & TEA & 300 & $\mathrm{ACOH}$ & Anlsole & 4.00 & 110 & 240 & $1.50 \%$ & $51.32 \%$ \\
\hline 10 & 10.0 & PdIOAC $)^{2}$ & DMSO & 200 & DAECO & 300 & $\mathrm{ACOH}$ & Anisole & 4.00 & 110 & 240 & $0.00 \%$ & $28.57 \%$ \\
\hline 10 & 10.0 & PdiOAc) 12 & DMSO & 200 & DIPEA & 300 & $\mathrm{ACOH}$ & Anisole & 4.00 & 110 & 240 & $1.80 \%$ & $54.84 \%$ \\
\hline 10 & 10.0 & PdIOAC) 2 & DMSO & 200 & Ктом⿻ & 300 & $\mathrm{ACOH}$ & Anisole & 4.00 & 110 & 240 & $0.00 \%$ & $58.39 \%$ \\
\hline 10 & 10.0 & PdIOAC) 2 & DMSO & 200 & 2,6-lubdine & 300 & $\mathrm{ACOH}$ & Arisole & 4.00 & 110 & 240 & $0.00 \%$ & $59.97 \%$ \\
\hline 10 & 10.0 & PdiOAc)12 & DMso & 200 & DMAP & 300 & $\mathrm{ACOH}$ & Anisole & 4.00 & 110 & 240 & $0.00 \%$ & $66.12 \%$ \\
\hline 10 & 10.0 & PdiOAc]2 2 & DMSO & 200 & DEU & 300 & $\mathrm{ACOH}$ & Anlsole & 4.00 & 110 & 240 & $0.00 \%$ & $67.79 \%$ \\
\hline 10 & 10.0 & PdiOAC) 2 & DMSO & 200 & CyzNME & 3.00 & $\mathrm{ACOH}$ & Anloole & 4.00 & 110 & 240 & $0.00 \%$ & $49.83 \%$ \\
\hline
\end{tabular}




\begin{tabular}{|c|c|c|c|c|c|c|c|c|c|c|c|c|c|c|}
\hline mol & $\begin{array}{l}\text { Esotrophio } \\
10 \text { equth }\end{array}$ & & $\begin{array}{l}\text { zoditive } \\
6 \mathrm{~mol} \%\end{array}$ & $\begin{array}{l}\text { Arocitive } \\
\text { Eguly }\end{array}$ & & & Upond & Arsaitive & ame & & amp & & Pot: & $\operatorname{SM}$ \\
\hline $\begin{array}{ll}710 \\
10\end{array}$ & $\begin{array}{l}\text { losobenzere } \\
\text { lodobenzere }\end{array}$ & 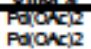 & $\begin{array}{l}\text { DMPU } \\
\text { DNPU }\end{array}$ & 4 & 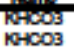 & $\begin{array}{l}300 \\
300\end{array}$ & 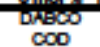 & ACOH & $\begin{array}{l}\text { Diriorane } \\
\text { Dloxane }\end{array}$ & $\begin{array}{l}4000 \\
400\end{array}$ & $\begin{array}{l}710 \\
110\end{array}$ & 2440 & 262256 & 522986 \\
\hline $\begin{array}{l}10 \\
10\end{array}$ & $\begin{array}{l}\text { lodobenzere } \\
\text { lodobenzere }\end{array}$ & $\begin{array}{l}\text { PaIOAC;12 } \\
\text { PdIOAC, } 2\end{array}$ & DMPU & 6 & $\begin{array}{l}\text { KHCOS } \\
\text { KHCO3 }\end{array}$ & $\begin{array}{l}300 \\
300\end{array}$ & $\begin{array}{l}\text { Pyildine } \\
\text { DeU }\end{array}$ & $\begin{array}{l}\mathrm{ACOH} \\
\mathrm{ACOH}\end{array}$ & $\begin{array}{l}\text { Dloxane } \\
\text { Dloxane }\end{array}$ & $\begin{array}{l}400 \\
400\end{array}$ & $\begin{array}{l}110 \\
110\end{array}$ & 24.0 & $\begin{array}{l}90.25 \% \\
7.55 \%\end{array}$ & $\begin{array}{l}0.04851 \\
0.8506\end{array}$ \\
\hline 10 & lodobenzere & PaIOAC:12 & & & $\mathrm{NHCO3}$ & & DABCO & $\mathrm{ACOH}$ & Avisole & 400 & 110 & 24.0 & $58.30 \%$ & 0.2029 \\
\hline 10 & lodoberizene & ParoAc)2 & & & NHCO3 & 300 & $\infty 00$ & $\mathrm{ACOH}$ & Avisole & 400 & 110 & 24.0 & 0 & $44.18 \%$ \\
\hline 10 & lodobenzere & PalOAC:2 & & & $\mathrm{NHCO3}$ & 300 & Pyridine & $\mathrm{ACOH}$ & Avisole & 400 & 110 & 24.0 & 1 & $18.81 \%$ \\
\hline 10 & lodobenzere & PaIOAC)/2 & & & $\mathrm{KHCO} 3$ & 300 & DeU & $\mathrm{ACOH}$ & Avisole & 400 & 110 & 24.0 & $1321 \%$ & $45.22 \%$ \\
\hline 10 & Bromoberzene & Paloncl2 2 & & & $\mathrm{NOCOS}$ & 300 & DABCO & $\mathrm{ACOH}$ & Dloxane & 400 & 110 & 24.0 & $0.00 \%$ & $48.61 \%$ \\
\hline 10 & Bromoberzene & PdIOAC)2 & & & $\mathrm{NHCO3}$ & 300 & $\infty 00$ & $\mathrm{NCOH}$ & Dloxane & 400 & 110 & 24.0 & $0.00 \%$ & $51.70 \%$ \\
\hline 10 & Bromoberzizene & PaIOAC) 2 & & & $\mathrm{HHCOS}$ & 300 & Pylidine & $\mathrm{ACOH}$ & Dloxane & 400 & 110 & 24.0 & $0.00 \%$ & $65.24 \%$ \\
\hline 10 & Bromoberzene & PdOCACI2 & & & $\mathrm{HOHCO}$ & 300 & Deu & $\mathrm{ACOH}$ & Dloxane & 400 & 110 & 24.0 & $2.40 \%$ & $48.18 \%$ \\
\hline $\begin{array}{l}710 \\
10\end{array}$ & $\begin{array}{l}\text { Todobenzere } \\
\text { lodoberzere }\end{array}$ & 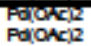 & $\begin{array}{l}\text { Wink } \\
\text { DMNC }\end{array}$ & $\begin{array}{l}2 \\
4\end{array}$ & $\begin{array}{l}\mathrm{RHCOOS} \\
\mathrm{NHCOS}\end{array}$ & $\begin{array}{l}300 \\
300\end{array}$ & $\begin{array}{l}\text { IPFARC } \\
\text { Phapoes }\end{array}$ & $\begin{array}{l}\text { RCOOH } \\
\mathrm{ACOH}\end{array}$ & $\begin{array}{l}\text { Diorasne } \\
\text { Dloxane }\end{array}$ & $\begin{array}{l}200 \\
400\end{array}$ & $\begin{array}{l}710 \\
110\end{array}$ & 24.0 & $\begin{array}{l}26.12 \% \\
60.32 \%\end{array}$ & $\begin{array}{l}54.65 \% \\
28.32 \%\end{array}$ \\
\hline 10 & lodobenzere & PaIOAC) 12 & DMAN & 6 & $\mathrm{NHCO} 3$ & 300 & the & $\mathrm{ACOH}$ & Dloxane & 400 & 110 & 24.0 & $8827 \%$ & $5.56 \%$ \\
\hline 10 & lodobenzere & PaIOAC:2 & & & $\mathrm{NHCO3}$ & 300 & 2,6-utiden & $\mathrm{ACOH}$ & Dloxane & 400 & 110 & 24.0 & $88.65 \%$ & $5.00 \% 6$ \\
\hline 10 & lodobenzere & PaIOACI2 & & & $\mathrm{NHCOS}$ & 300 & IPAHC & $\mathrm{NCOH}$ & Avisole & 400 & 110 & 24.0 & $13.36 \%$ & $6244 \%$ \\
\hline 10 & lodobenzene & PaloAci2 & & & $\mathrm{HHCO3}$ & 300 & Phopoes & $\mathrm{ACOH}$ & Avisole & 400 & 110 & 24.0 & $55.56 \%$ & $2216 \mathrm{~N}$ \\
\hline 10 & lodobenzere & PaIOAC):2 & & & $\mathrm{NHCO}$ & 300 & thes & $\mathrm{ACOH}$ & Anisole & 400 & 110 & 24.0 & $7359 \%$ & $17.35 \%$ \\
\hline 10 & lodobenzere & PaIOAC)12 & & & $\mathrm{NHCO3}$ & 300 & 2,6-utiden & $\mathrm{ACOH}$ & Arisole & 400 & 110 & 24.0 & $60.96 \%$ & $18.95 \%$ \\
\hline 10 & Bromoberzene & PalOAC)12 & & & $\mathrm{NHCO3}$ & 300 & IPNHC & $\mathrm{ACOH}$ & Dloxane & 400 & 110 & 24.0 & $0.00 \%$ & $49.30 \%$ \\
\hline 10 & Bromoberzene & PaIOAC: 12 & & & $\mathrm{NHCOS}$ & 300 & Pnapoet & $\mathrm{ACOH}$ & Dloxane & 400 & 110 & 24.0 & $0.00 \%$ & $61.49 \% 6$ \\
\hline 10 & Bromoberzizene & PaIOACI2 & & & $\mathrm{KHCO} 3$ & 300 & tos & $\mathrm{ACOH}$ & Dloxane & 400 & 110 & 24.0 & $0.13 \%$ & $54.83 \%$ \\
\hline 10 & Bromoberzene & PaIOAC:12 & & & $\mathrm{NHCO3}$ & 3.00 & 2,6-utiden & $\mathrm{ACOH}$ & Dloxane & 400 & 110 & 24.0 & $0.00 \%$ & $50.21 \%$ \\
\hline $\begin{array}{l}70 \\
10\end{array}$ & $\begin{array}{l}\text { Todoochizene } \\
\text { lodobentere }\end{array}$ & $\begin{array}{l}\text { Paxorel2 } \\
\text { PdOACI2 }\end{array}$ & $\begin{array}{l}\text { TMM- } \\
\text { Nape }\end{array}$ & 2 & $\begin{array}{l}\mathrm{RHCOOS} \\
\mathrm{PHCO} 3\end{array}$ & 300 & phatu & NCOH & Diroxane & 400 & 710 & 240 & $23.63 \%$ & $68.50 \%$ \\
\hline 10 & lodobenzere & PaIOACI2 & NMP & 6 & $\mathrm{NOCOO}$ & 300 & styrene & $\mathrm{ACOH}$ & Dloxane & 400 & 110 & 24.0 & $9061 \%$ & $4.39 \%$ \\
\hline 10 & lodobenzere & PaIOAC:2 & & & $\mathrm{NHCOS}$ & 300 & DFPE & $\mathrm{NCOH}$ & Dloxane & 400 & 110 & 24.0 & $9003 \%$ & $541 \%$ \\
\hline 10 & lodobenzere & PdOAC, 2 & & & NHCO3 & 300 & stau & $\mathrm{NCOH}$ & Avisole & 400 & 110 & 24.0 & $21.85 \%$ & $55.68 \%$ \\
\hline 10 & lodobenzere & PaIOAC:2 & & & $\mathrm{NHCO} 3$ & 300 & $P(O t a)_{3}$ & $\mathrm{ACOH}$ & Anisole & 400 & 110 & 24.0 & $73.37 \%$ & $2022 \%$ \\
\hline 10 & lodobenzere & PalOAC)/2 & & & $\mathrm{KHCO} 3$ & 300 & styrene & $\mathrm{ACOH}$ & Avisole & 400 & 110 & 24.0 & $5265 \%$ & $25.58 \%$ \\
\hline 10 & lodobenzere & PaIOAC)/2 & & & $\mathrm{NHCO3}$ & 300 & DFPE & $\mathrm{ACOH}$ & Avisole & 400 & 110 & 24.0 & $49.59 \%$ & $30.70 \%$ \\
\hline 10 & Bromoberzzene & PaIOAC: 12 & & & $\mathrm{NHCO3}$ & 300 & stau & $\mathrm{ACOH}$ & Dloxane & 400 & 110 & 24.0 & $0.00 \%$ & $68.95 \%$ \\
\hline 10 & Bromoberzene & PaIOAC:2 & & & $\mathrm{NHCO} 3$ & 300 & P(Ota)3 & $\mathrm{ACOH}$ & Dloxane & 400 & 110 & 24.0 & $0.00 \%$ & $69.69 \%$ \\
\hline $\begin{array}{l}10 \\
10\end{array}$ & $\begin{array}{l}\text { Eromoberzizene } \\
\text { Bromoberzene }\end{array}$ & $\begin{array}{l}\text { PaIOACl2 } \\
\text { PdOACI2 }\end{array}$ & & & $\begin{array}{l}\mathrm{NHCOS} \\
\mathrm{NHCOS}\end{array}$ & $\begin{array}{r}300 \\
300 \\
\end{array}$ & $\begin{array}{l}\text { styrene } \\
\text { DFPE }\end{array}$ & $\underset{\mathrm{ACOH}}{\mathrm{ACOH}}$ & $\begin{array}{l}\text { Dloxane } \\
\text { Dlowane }\end{array}$ & $\begin{array}{l}400 \\
400\end{array}$ & $\begin{array}{l}110 \\
110\end{array}$ & $\begin{array}{l}24.0 \\
24.0\end{array}$ & $\begin{array}{l}0.54 \% \\
0.00 \%\end{array}$ & $\begin{array}{l}51.97 \% \\
45.05 \%\end{array}$ \\
\hline 70 & Todooenzere & paracels & Denso & 2 & RACOOS & 3000 & $\rightarrow=\mathrm{XDC}$ & RCOA & wioxane & 4000 & $170^{\circ}$ & 240 & $59.30 \%$ & $35.49 \% 6$ \\
\hline 10 & lodobenzzere & Paloacl2 & Duso & 4 & $\mathrm{NHCO3}$ & 300 & $P(O F P(x) 3$ & $\mathrm{ACOH}$ & Dloxane & 400 & 110 & 24.0 & $2898 \%$ & $60.22 \%$ \\
\hline 10 & lodobenzere & PaloAC:2 & Duso & 6 & $\mathrm{NHCOS}$ & 300 & $2 y=$ atyen & $\mathrm{ACOH}$ & Dloxasne & 400 & 110 & 24.0 & $2320 \%$ & $61.30 \%$ \\
\hline 10 & lodobenizere & PdOACI2 & & & $\mathrm{NHCO3}$ & 300 & CFSP & $\mathrm{ACOH}$ & Dloxane & 400 & 110 & 24.0 & $30.65 \%$ & $57.40 \%$ \\
\hline 10 & lodobenzere & Paloncl2 & & & $\mathrm{NHCO3}$ & 300 & FACAC & $\mathrm{ACOH}$ & Avisole & 400 & 110 & 24.0 & $46.35 \%$ & $35.74 \%$ \\
\hline 10 & lodobenzere & PaIOAC:12 & & & $\mathrm{NHCO3}$ & 300 & $P(O F+r)^{3}$ & $\mathrm{ACOH}$ & Avisole & 400 & 110 & 24.0 & $6432 \%$ & $19.16 \%$ \\
\hline 10 & lodobenzere & PaIOACi2 & & & $\mathrm{NHCOS}$ & 300 & $>3 \Rightarrow$ atren & $\mathrm{ACOH}$ & Avisole & 400 & 110 & 24.0 & $54.68 \%$ & $23.95 \%$ \\
\hline 10 & lodobenzere & ParoAcl2 & & & $\mathrm{NHCO} 3$ & 300 & CFYP & $\mathrm{ACOH}$ & Avisole & 400 & 110 & 24.0 & $26.15 \%$ & $4722 \%$ \\
\hline 10 & Bromoberzene & PdOACI2 & & & $\mathrm{NHCO3}$ & 300 & FACAC & $\mathrm{ACOH}$ & Dloxane & 400 & 110 & 24.0 & $0.00 \%$ & $65.84 \%$ \\
\hline 10 & Bromoberzzene & Parons:12 & & & $\mathrm{NHCO3}$ & 300 & $P(O F P r)^{3}$ & $\mathrm{ACOH}$ & Dloxane & 400 & 110 & 24.0 & $0.00 \%$ & $54.18 \%$ \\
\hline 10 & Bromoberzene & PdYOAC)2 & & & $\mathrm{OHCO3}$ & 300 & zryetaren & $\mathrm{ACOH}$ & Dloxane & 400 & 110 & 24.0 & $0.00 \%$ & $49.15 \%$ \\
\hline 10 & Bromoberzzene & PdIOACI2 & & & $\mathrm{NHCO} 3$ & 300 & CF $P$ & $\mathrm{ACOH}$ & Dioxane & 400 & 110 & 24.0 & $0.15 \%$ & $64.53 \%$ \\
\hline
\end{tabular}




\begin{tabular}{|c|c|c|c|c|c|c|c|c|c|c|c|c|c|c|}
\hline \multirow{2}{*}{$\begin{array}{l}\text { SMT } \\
\text { ymol }\end{array}$} & \multirow{2}{*}{$\begin{array}{l}\text { Electrophise } \\
\text { to equit }\end{array}$} & \multirow{2}{*}{$\begin{array}{c}1 \mathrm{Nd} \\
5 \mathrm{~mol} / \mathrm{s}\end{array}$} & \multirow{2}{*}{$\begin{array}{l}\text { Adalave } \\
5 \mathrm{~mol} \gamma\end{array}$} & \multirow{2}{*}{$\begin{array}{l}\text { Adeditive } \\
\text { Equely }\end{array}$} & \multicolumn{2}{|c|}{ Gesese } & \multirow{2}{*}{$\begin{array}{l}\text { पt } 5 \text { and } \\
5 \text { mol } 4\end{array}$} & \multirow{2}{*}{ 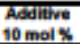 } & \multirow[b]{2}{*}{ Name } & \multirow{2}{*}{\multicolumn{3}{|c|}{ Temp }} & \multirow{2}{*}{ TAP } & \multirow{2}{*}{${ }_{A P}^{B M}$} \\
\hline & & & & & Neme & Eque & & & & nol & & h & & \\
\hline טv & 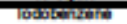 & Pacorats & DOADE & & RAFcus & soo & हक्लन्रा & DEOA & एक्यान & 200 & Tho & 200 & $50.75 \times 5$ & $58716 x$ \\
\hline 10 & lodobenzate & Pacosel? & DNAC & 4 & $\mathrm{KHCOS}$ & 900 & PqONejs & $\mathrm{ACOH}$ & Diecane & 4.00 & 110 & 240 & $45.65 \%$ & $41.34 \%$ \\
\hline 10 & lodotenzinte & Pacouclo & DNAC & e & $\mathrm{KHCOS}$ & 900 & CF9-pr & $\mathrm{ACOH}$ & Dicuane & 4.00 & 110 & 240 & $19.46 \%$ & $65.37 \%$ \\
\hline 10 & lodobenzate & Pacose? & DNAC & 2 & $\mathrm{KHCOS}$ & 900 & PMes & $\mathrm{AeOH}$ & Dicuane & 400 & 110 & 240 & 49.7046 & $44 \cos$ \\
\hline 10 & lodotemzete & PdOACl2 & DNAC & 2 & $\mathrm{KHCOS}$ & 900 & suspr & $\mathrm{ACOH}$ & Arisole & 4.00 & 110 & 240 & $1901 \%$ & $50290 x$ \\
\hline 10 & lodotenzante & PaCACL2 & DNAC & 2 & KHCOS & 900 & POONe]S & $\mathrm{ACOH}$ & Arisole & 4.00 & 110 & 240 & $28.47 \%$ & $40000 \mathrm{x}$ \\
\hline 10 & lodobenzete & PdoAcl? & DWAC & 2 & $\mathrm{KHCOS}$ & 300 & $C P 9-p y r$ & $\mathrm{AEOH}$ & Aniscie & 4.00 & 110 & 240 & $59.40 \%$ & $30.13 \%$ \\
\hline 10 & lodobenzate & Pachele? & DNAC & 2 & $\mathrm{KHCOS}$ & 900 & PNes & $\mathrm{ACOH}$ & Ariscle & 4.00 & 110 & 240 & $40.27 \mathrm{~s}$ & $97.52 \%$ \\
\hline 10 & 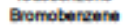 & Palosel2 & DNAC & 2 & $\mathrm{KHCOS}$ & 900 & surgr & $\mathrm{ACOH}$ & Dicuane & 400 & 110 & 240 & $0.00 \%$ & $44.52 \%$ \\
\hline 10 & Bromotenzate & Pdoucle & DNAC & 2 & $\mathrm{KHCOS}$ & 900 & PiONo) & $\mathrm{AEOH}$ & Dimoane & 400 & 110 & 240 & $0.00 \%$ & $63.07 \%$ \\
\hline 10 & Gromblenzente & PaOAcp & DNAC & 2 & $\begin{array}{l}\mathrm{KHCOS} \\
\mathrm{KHCOS}\end{array}$ & 900 & cFy-pr & $\mathrm{AeOH}$ & Diwasene & 400 & 110 & 240 & $0.00 \%$ & 402004 \\
\hline & 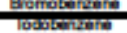 & 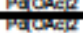 & $\frac{10 N A C}{10 N}$ & $\frac{2}{200}$ & $\frac{\text { KACCOS }}{\text { Kateus }}$ & $\frac{100}{500}$ & Poves & $\frac{A C O H}{2 E O H A}$ & $\begin{array}{l}\text { Dowane } \\
\text { Lumaln }\end{array}$ & 4.00 & $\frac{110}{7 \times 10}$ & 24.0 & $\begin{array}{l}0.00 \% \\
59.76 \%\end{array}$ & $\begin{array}{l}61.505 \% \\
40.05 \%\end{array}$ \\
\hline 10 & lodotemate & PdOAC:2 & Nap & 400 & $\mathrm{KHCOS}$ & 900 & Pners & $\widehat{A} \mathrm{COH}$ & Dimane & 4.00 & 110 & 240 & $2895 \%$ & $5237 \%$ \\
\hline 10 & lodobenzate & Pachele & NaP & $0 \infty$ & KHCOS & 900 & CMe-pyr & $\mathrm{ACOH}$ & Diware & 4.00 & 110 & 240 & $19.72 \times$ & 64.07\% \\
\hline 10 & lodobemzate & Páacel? & NaE & 200 & $\mathrm{KHCOS}$ & 900 & ADAe & $\mathrm{ACOH}$ & Dicuane & 400 & 110 & 240 & $18.85 \%$ & 64.4e\% \\
\hline 10 & lodotenzite & Pacher & nae & 200 & $\mathrm{KHCOS}$ & 900 & ONOL & $\mathrm{ACOH}$ & Anisole & 400 & 110 & 240 & $75.17 \%$ & $18.49 \%$ \\
\hline 10 & lodobenzare & Pacoselo & NaO & 200 & $\mathrm{KHCOS}$ & 900 & Pneus & $\mathrm{ACOH}$ & Anisole & 4.00 & 110 & 240 & $10.54 \%$ & $57.25 \%$ \\
\hline 10 & ledoberzene & Pacacis & NaP & 200 & KHCOS & 900 & CMno-pyr & $\mathrm{ACOH}$ & Anisole & 4.00 & 110 & 240 & 78.446 & $17.6 \mathrm{ax}$ \\
\hline 10 & ladebersene & Pachelo & nase & 200 & KHCOS & 900 & NOAE & $\mathrm{ACOH}$ & Avisole & 4.00 & 110 & 240 & $46.86 \%$ & $3287 \%$ \\
\hline 10 & Bromoberzene & Pacosel? & NaO & 200 & $\mathrm{KHCOS}$ & 900 & CNOL & $\mathrm{ACOH}$ & Dicuane & 400 & 110 & 240 & $0.00 \%$ & $88.42 \%$ \\
\hline 10 & Bromobercene & Pacosel? & NAP & 200 & $\mathrm{KHCOS}$ & 900 & Pnews & $\mathrm{ACOH}$ & Diocane & 4.00 & 110 & 240 & $0.05 \%$ & $58.03 \%$ \\
\hline 10 & Gomoterzene: & PaOACl? & NaP & 200 & KHCOS & 900 & CMe-pyr & $\mathrm{ACOH}$ & Diwane & 400 & 110 & 240 & $0.11 \%$ & 64.76\% \\
\hline 10 & Gemoterzente & PdoAcia & NMP & 200 & $\mathrm{KHCOS}$ & 300 & ADSE & $\mathrm{A}_{\mathrm{COOH}}$ & Dimane & 4.00 & 110 & 24.0 & $0.11 \%$ & $54.60 \mathrm{k}$ \\
\hline & 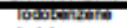 & Pateacer? & Douso & & RAcus & suov & $\operatorname{sen} x$ & DEUत & Uस्वान & 200 & & & $14.06 \%$ & 90.62\% \\
\hline 10 & lodotemzate & PaOACER & DNso & 400 & $\mathrm{KHCOS}$ & 900 & Bank. & $\mathrm{ACOH}$ & Diovane & 4.00 & 110 & 24.0 & $24.55 \%$ & $6200 \%$ \\
\hline 10 & lodotenzate & Pacosel? & DUso & 000 & $\mathrm{KHCOS}$ & 900 & Mepry & $\mathrm{ACOH}$ & Dicuane & 4.00 & 110 & 240 & $15.95 \%$ & $93.00 \%$ \\
\hline 10 & lodobenzate & PdoAclo & DUso & 200 & KHCOS & 900 & Bark. & $\mathrm{ACOH}$ & Diocane & 4.00 & 110 & 240 & 18064 & $55.52 \%$ \\
\hline 10 & lodobenzafe & Pacaclo & DNSO & 200 & KHCOS & 900 & Bank. & $\mathrm{ACOH}$ & Anisole & 400 & 110 & 240 & $48.06 \%$ & $44.04 \%$ \\
\hline 10 & lodotenzate & Pacoucl? & DNso & 200 & $\mathrm{KHCOS}$ & 900 & Bark. & $\mathrm{ACOH}$ & Anisole & 4.00 & 110 & 240 & 47. $45 \%$ & $3420 \mathrm{x}$ \\
\hline 10 & lodotemzate & PAOACER & DNso & 200 & $\mathrm{KHCOS}$ & 900 & Mepyr & $\mathrm{ACOH}$ & Anisole & 4.00 & 110 & 24.0 & $19.96 \%$ & 68.91\% \\
\hline 10 & lodotenzate & Pacher & DUso & 200 & $\mathrm{KHCOS}$ & 900 & Bark & $\mathrm{ACOH}$ & Anisole & 400 & 110 & 240 & 47006 & $95.00 \%$ \\
\hline 10 & Bromotefzente & Páace? & DNsO & 200 & $\mathrm{KHCOS}$ & 900 & Bank & $\mathrm{ACOH}$ & Dicuane & 400 & 110 & 240 & $0.00 \%$ & 4. Sex \\
\hline 10 & Exomotenzente & Pacoser & DNSO & 200 & $\mathrm{KHCOS}$ & 900 & Bark & $\mathrm{ACOH}$ & Diocane & 400 & 110 & 240 & $0.00 \%$ & $84.37 \%$ \\
\hline 10 & Eromobefzente & Pacouclo & DNSO & 200 & $\mathrm{KHCOS}$ & 900 & Mepy & $\mathrm{ACOH}$ & Dicuane & 4.00 & 110 & 240 & $0.00 \%$ & $5221 \%$ \\
\hline 10 & Gomotenzente & Pdoser & DNSO & 200 & $\mathrm{KHCOS}$ & 300 & Batik. & $\mathrm{ACOH}$ & Dimane & 4.00 & 110 & 24.0 & $0.00 \%$ & $61.00 \%$ \\
\hline & |ण0खा & prosede? & DINPOU & & Rateus & soo & 2-phuecori & DEUत' & पिएवाल & 200 & Tou & 2010 & $0.00 \%$ & $92.55 \%$ \\
\hline 10 & lodobenzente & PACACLP & DNOU & 4 & KHCOS & 900 & Picens & $\mathrm{ACOH}$ & Diware & 4.00 & 110 & 240 & $0.00 \%$ & 98.10x \\
\hline 10 & lodobenzate & Pacosep? & DNDU & 8 & $\mathrm{KHCOS}$ & 900 & overey & $\mathrm{ACOH}$ & Dicuane & 4.00 & 110 & 240 & $68.96 \%$ & $48 \mathrm{es}$ \\
\hline 10 & lodotenzate & PdOAC:2 & DNOU & 2 & $\mathrm{KHCOS}$ & 900 & DUso & $\mathrm{A} \in \mathrm{OH}$ & Dicoane & 4.00 & 110 & 240 & 87.294 & $4.70 \%$ \\
\hline 10 & lodobenzefo & Pacoselo & DNOU & 2 & KHCOS & 900 & 2-prar $\mathrm{CeCH}$ & $\mathrm{ACOH}$ & Anisole & 4.00 & 110 & 240 & 52386 & $27.60 \mathrm{~K}$ \\
\hline 10 & lodobunzate & Páacip & DNDU & 2 & $\mathrm{KHCOS}$ & 900 & Picens & $\mathrm{ACOH}$ & Aclisiole & 400 & 110 & 240 & $47.90 \%$ & $32.44 \%$ \\
\hline 10 & lodotemzete & PaOACER & ONPU & 2 & $\mathrm{KHCOS}$ & 900 & ove-ey & $\mathrm{ACOH}$ & Anisole & 4.00 & 110 & 24.0 & $52.47 \%$ & $2867 \%$ \\
\hline 10 & lodotenzatio & Pacoselo & DNOU & 2 & $\mathrm{KHCOS}$ & 900 & Duso' & $\mathrm{ACOH}$ & Anisole & 400 & 110 & 240 & $50.76 \%$ & $2850 \%$ \\
\hline 10 & Exomoteratere & Pachel? & DNOU & 2 & KHCOS & 900 & 2-pruech & $\mathrm{ACOH}$ & Diwane & 4.00 & 110 & 240 & $0.00 \%$ & $50.07 \%$ \\
\hline 10 & Exomoberzane & Pacoselo & DNOU & 2 & KHCOS & 900 & Proess & $\mathrm{ACOH}$ & Diware & 4.00 & 110 & 240 & $0.00 \%$ & $53.73 \%$ \\
\hline 10 & Bromotenzente & Pdicacis & DNOU & 2 & $\mathrm{KHCOS}$ & 900 & oule-ky & $\mathrm{ACOH}$ & Dicuane & 4.00 & 110 & 240 & $0.00 \%$ & $51.84 \%$ \\
\hline 10 & Bromotenzante & Pdione? & DNOU & 3 & $\mathrm{KHCOS}$ & 300 & DUSO' & $\mathrm{A} \in \mathrm{OH}$ & Dimane & 4.00 & 110 & 24.0 & $0.00 \%$ & $55.17 \%$ \\
\hline
\end{tabular}

Standard conditions: 5 mol \% PdiOAc)2, 3.0 equilv base, 10 mol \% pyridline, 5/3 Anlsole/Phl (8 vol total]

\begin{tabular}{|c|c|c|}
\hline Variation & sm & Produot \\
\hline KOAC & 43.40 & 40.82 \\
\hline KOPIV & 0.22 & 80.88 \\
\hline CaOAC & 2.95 & 46.36 \\
\hline NaOAc & 85.26 & 7.65 \\
\hline K-Ethylhexanoate & 0.19 & 58.00 \\
\hline CsOPIv $(7.5 \mathrm{~h})$ & 0.70 & 51.63 \\
\hline AgOAC & 41.97 & 4.58 \\
\hline 1 equlv KOPlv & 9.64 & 72.53 \\
\hline 3 equlv KOPlv, open to air & 0.04 & 72.53 \\
\hline 3 equiv CsOAC, $110 \mathrm{C}$ (batch 1) & 0.02 & 56.33 \\
\hline 3 equiv CsOAC, $110 \mathrm{C}$ (bottch 2) & 0.05 & 60.86 \\
\hline 1 equiv CsOAC, 95 C (batch 1) & 77.42 & 21.38 \\
\hline 1 equiv CsOAC, $95 \mathrm{C}$ (batch 2) & 78.18 & 20.19 \\
\hline $0.50 \mathrm{Cs} 2 \mathrm{CO} 3,1.05 \mathrm{PlNOH}$ & 0.02 & 87.01 \\
\hline $0.50 \mathrm{Cs} 2 \mathrm{CO} 3,1.05 \mathrm{PWOH}$ & 0.02 & 84.92 \\
\hline $0.50 \mathrm{Cs} 2 \mathrm{CO} 3,1.25 \mathrm{PlNOH}$ & 0.02 & 86.88 \\
\hline $0.47 \mathrm{Cs} 2 \mathrm{CO} 3,1.00 \mathrm{PlNOH}$ & 5.80 & 84.05 \\
\hline $0.53 \mathrm{Cs} 2 \mathrm{CO} 3,1.00 \mathrm{PlvOH}$ & 0.02 & 79.84 \\
\hline $0.53 \mathrm{Cs} 2 \mathrm{CO} 3,1.11 \mathrm{Pl}$ OH & 0.05 & 85.36 \\
\hline Amount of wator & 84 & Produot \\
\hline 1 EquV & 3.19 & 82.98 \\
\hline 3 equiv & 46.36 & 43.52 \\
\hline 5 equiv & 62.50 & 30.25 \\
\hline
\end{tabular}

\begin{tabular}{|c|c|c|}
\hline Variation & $\mathbf{s m}$ & Produot \\
\hline 1.05 equil CeOAc $-110 \mathrm{C}$ & 0.90 & 88.55 \\
\hline 2 equiv CsOAc-95 C & 82.47 & 14.11 \\
\hline 3 equiv KOPlv - 2 MeOETOH (solv) & 58.29 & 32.95 \\
\hline 1.05 equiv CaOAC - $2 \mathrm{MeOE}$ (OOH (solv) & 65.23 & 24.44 \\
\hline $\operatorname{KOBz}(1$ equiv) & 59.65 & 18.59 \\
\hline KOBz (2 equiv) & 69.72 & 10.76 \\
\hline KOBz (3 equlv) & 71.90 & 11.08 \\
\hline KOez (1 equlv) + pyr & 74.06 & 9.76 \\
\hline KOPIV (1 equiv) & 0.51 & 78.99 \\
\hline KOPIV (1 equiv)+pyr & 1.53 & 74.29 \\
\hline KOPW (2 equiv) & 0.81 & 72.81 \\
\hline KOPlv (2 equiv) + pyr & 0.50 & 77.47 \\
\hline KOPlv (3 equiv) & 1.99 & 79.98 \\
\hline KOPliv (3 equiv) + pyr & 0.14 & 80.85 \\
\hline$y m e(s o l v) \quad K$ & 44.97 & 39.17 \\
\hline
\end{tabular}

\begin{tabular}{ccc} 
Amountof & sm & Produot \\
\hline 0.00 & 2.3 .94 & 65.12 \\
0.05 & 0.00 & 78.95 \\
0.10 & 0.04 & 86.02 \\
0.20 & 0.07 & 83.12 \\
0.50 & 12.79 & 75.93 \\
1.00 & 27.15 & 63.24
\end{tabular}




\begin{tabular}{|c|c|c|c|c|c|c|c|c|c|c|c|c|}
\hline $\begin{array}{l}\text { Reaction } \\
\text { ID }\end{array}$ & $\begin{array}{l}\text { Arene } \\
\text { Jumol }\end{array}$ & $\begin{array}{l}\text { Phl } \\
\text { equlv }\end{array}$ & $\begin{array}{l}\mathrm{Pd}(\mathrm{OAC}) 2 \\
\mathrm{~mol} \%\end{array}$ & $\begin{array}{l}\mathrm{Cs} 2 \mathrm{CO} 3 \\
\text { equily }\end{array}$ & $\begin{array}{l}\text { PlwOH } \\
\text { egulv }\end{array}$ & $\begin{array}{l}\text { Water } \\
\text { equlv }\end{array}$ & KF (wts) & $\begin{array}{l}\text { Solvent } \\
\text { Name }\end{array}$ & wol & $\begin{array}{l}\text { Temp } \\
{ }^{\circ} \mathrm{C}\end{array}$ & $\begin{array}{l}\text { Prod } \\
\text { AP }\end{array}$ & $\begin{array}{l}\text { SM } \\
\text { AP }\end{array}$ \\
\hline A1 & 497.19 & 9.05 & 0.07 & 0.51 & 1.01 & 302 & 1.01 & Anlsole & 4.02 & 106 & $52.54 \%$ & $46.97 \%$ \\
\hline A2 2 & 502.32 & 8.96 & 0.03 & 0.57 & 1.14 & 3.00 & 1.14 & Anisole & 3.98 & 108 & $58.45 \%$ & $40.69 \%$ \\
\hline$A_{3}$ & 502.75 & 14.92 & 0.03 & 0.53 & 1.00 & 1.01 & 1.00 & Anlsole & 3.98 & 108 & $96.90 \%$ & \\
\hline A4 & 496.97 & 15.09 & 0.03 & 0.50 & 1.29 & 3.02 & 1.29 & Anlsole & 6.04 & 108 & $96.80 \%$ & $0.79 \%$ \\
\hline A5 & 496.12 & 9.07 & 0.03 & 0.55 & 1.01 & 1.01 & 1.01 & Anlsole & 6.05 & 108 & $58.28 \%$ & $41.00 \%$ \\
\hline A6 & 501.47 & 14.96 & 0.07 & 0.50 & 1.33 & 1.01 & 1.33 & Anlsole & 3.99 & 108 & $93.96 \%$ & \\
\hline B1 & 496.76 & 15.10 & 0.07 & 0.56 & 1.26 & 3.02 & 1.26 & Anlsole & 6.04 & 108 & $96.40 \%$ & \\
\hline $\begin{array}{l}82 \\
83\end{array}$ & $\begin{array}{l}496.54 \\
504.25\end{array}$ & $\begin{array}{l}15.10 \\
14.87\end{array}$ & $\begin{array}{l}0.03 \\
0.07\end{array}$ & $\begin{array}{l}0.59 \\
0.58\end{array}$ & $\begin{array}{l}1.31 \\
0.99\end{array}$ & $\begin{array}{l}1.01 \\
299\end{array}$ & $\begin{array}{l}1.31 \\
0.99\end{array}$ & $\begin{array}{l}\text { Anlsole } \\
\text { Anlsole }\end{array}$ & $\begin{array}{l}4.03 \\
5.95\end{array}$ & $\begin{array}{l}108 \\
108\end{array}$ & $\begin{array}{l}96.07 \% \\
98.05 \%\end{array}$ & $0.13 \%$ \\
\hline B4 & 496.54 & 9.06 & 0.07 & 0.58 & 1.12 & 1.01 & 1.12 & Anlsole & 6.04 & 108 & $61.36 \%$ & $38.11 \%$ \\
\hline B5 & 498.68 & 1203 & 0.05 & 0.58 & 1.16 & 201 & 1.16 & Anisole & 5.01 & 119 & $94.95 \%$ & \\
\hline 86 & 504.03 & 11.90 & 0.05 & 0.54 & $\begin{array}{r}1.10 \\
1.15\end{array}$ & 200 & $\begin{array}{l}1.10 \\
1.15\end{array}$ & Anisole & 4.96 & 119 & $94.91 \%$ & \\
\hline C1 & 499.54 & 1201 & 0.05 & 0.60 & 1.15 & 201 & 1.15 & Anlsole & 5.00 & 119 & $95.21 \%$ & \\
\hline$C_{2}$ & 500.18 & 1200 & 0.05 & 0.66 & 1.10 & 201 & 1.10 & Anlsole & 5.00 & 119 & $94.55 \%$ & \\
\hline C3 & 497.19 & 15.08 & 0.07 & 0.51 & 1.01 & 1.01 & 1.01 & Anlsole & 6.04 & 130 & $93.25 \%$ & \\
\hline$C 4$ & 504.68 & 14.86 & 0.03 & 0.58 & 1.04 & 299 & 1.04 & Anlsole & 3.96 & 130 & $94.98 \%$ & \\
\hline $\begin{array}{l}C 5 \\
C 6\end{array}$ & $\begin{array}{l}499.75 \\
501.68\end{array}$ & $\begin{array}{l}15.01 \\
8.97\end{array}$ & $\begin{array}{l}0.03 \\
0.03\end{array}$ & $\begin{array}{l}0.56 \\
0.57\end{array}$ & $\begin{array}{l}1.12 \\
1.35\end{array}$ & $\begin{array}{l}1.01 \\
1.01\end{array}$ & $\begin{array}{l}1.12 \\
1.35\end{array}$ & $\begin{array}{l}\text { Anlsoie } \\
\text { Anlsole }\end{array}$ & $\begin{array}{l}6.00 \\
3.99\end{array}$ & $\begin{array}{l}130 \\
130\end{array}$ & $\begin{array}{l}93.68 \% \\
94.04 \%\end{array}$ & $2.09 \%$ \\
\hline D1 & 501.47 & 8.97 & 0.07 & 0.52 & 1.03 & 1.01 & 1.03 & Anlsole & 3.99 & 130 & $91.67 \%$ & \\
\hline$D 2$ & 503.39 & 14.90 & 0.07 & 0.66 & 1.13 & 3.00 & 1.13 & Anlsole & 3.97 & 130 & $84.04 \%$ & \\
\hline D3 & 500.61 & 8.99 & 0.07 & 0.61 & 1.29 & 1.01 & 1.29 & Anlsole & 3.99 & 130 & $90.81 \%$ & \\
\hline D4 & 495.69 & 9.08 & 0.03 & 0.59 & 1.09 & 3.03 & 1.09 & Anlsole & 6.05 & 130 & $95.01 \%$ & \\
\hline D5 & 499.75 & 9.00 & 0.03 & 0.57 & 1.27 & 3.01 & 1.27 & Anlsole & 6.00 & 130 & $96.64 \%$ & \\
\hline D6 & 499.33 & 9.01 & 0.07 & 0.68 & 1.49 & 3.01 & 1.49 & Anlsole & 6.01 & 130 & $92.88 \%$ & \\
\hline
\end{tabular}




\section{Computational Study}

Computational methods. DFT calculations were performed using the Gaussian $09{ }^{1}$ program. Geometry optimizations and frequency calculations in the gas phase were carried out with the hybrid exchange functional ${\mathrm{B} 3 L Y \mathrm{P}^{2}}^{2}$ and the TZVPP basis set, ${ }^{3}$ including revised def 2 effective core potentials (ECP) for the Pd, and I atoms. The energies showed herein correspond to free energies calculated at $298.15 \mathrm{~K}$ and $1 \mathrm{~atm}$ pressure. The stationary points were characterized as minima by the absence of imaginary frequencies, while the transition states were confirmed to have a single imaginary frequency that matched the change in geometry in going from reactants to products.

\begin{tabular}{|c|c|c|c|c|c|c|c|}
\hline \multicolumn{4}{|c|}{ Acetic Acid (-228.866797 Hartree) } & & & & \\
\hline \multicolumn{4}{|c|}{ C $-0.093123 \quad 0.126180 \quad 0.000008$} & & & & \\
\hline \multicolumn{4}{|c|}{ C $1.396442 \quad-0.114423 \quad 0.000007$} & & & & \\
\hline \multicolumn{4}{|c|}{$\begin{array}{llll}O & -0.639202 & 1.199712 & -0.000003\end{array}$} & & & & \\
\hline \multicolumn{4}{|c|}{ O $-0.783293 \quad-1.038161-0.000008$} & & & & \\
\hline \multicolumn{4}{|c|}{ H $\quad \begin{array}{lll}1.925513 & 0.849074 & -0.000070\end{array}$} & & & & \\
\hline \multicolumn{4}{|c|}{ H $1.683733 \quad-0.703884 \quad-0.888272$} & & & & \\
\hline \multicolumn{4}{|c|}{ H $\quad 1.683850 \quad-0.703802 \quad 0.888278$} & & & & \\
\hline \multicolumn{4}{|c|}{$\begin{array}{llll}H & -1.733047 & -0.804336 & 0.000060 \\
\end{array}$} & & & & \\
\hline \multicolumn{4}{|c|}{ 3d (-885.704965 Hartree) } & \multicolumn{4}{|c|}{ 3f (-1414.915057 Hartree) } \\
\hline & -2.512126 & -2.183909 & -0.011980 & & $0.612532-2$ & -2.713505 & 0.878052 \\
\hline & -1.598241 & -1.141865 & 0.026115 & & $1.157943-$ & -1.504237 & 0.462396 \\
\hline & -2.098516 & 0.179557 & 0.046660 & C & $2.496443-$ & -1.521907 & -0.004080 \\
\hline & -3.469256 & 0.503392 & 0.011504 & $\mathrm{C}$ & $3.292939-2$ & -2.684237 & -0.073702 \\
\hline & -4.385947 & -0.582108 & -0.022406 & & $2.702369-$ & -3.903192 & 0.354590 \\
\hline & -3.905267 & -1.883729 & -0.033675 & & $1.395730-3$ & -3.900681 & 0.816786 \\
\hline & -1.158814 & 1.260169 & 0.055392 & & $3.093274-($ & -0.286788 & -0.428858 \\
\hline & -3.856549 & 1.819866 & -0.013956 & $\mathrm{~N}$ & $4.581796-$ & -2.608534 & -0.539782 \\
\hline & 0.135762 & 0.863428 & 0.129670 & $\mathrm{~N}$ & 2.272824 & 0.777105 & -0.328238 \\
\hline & -1.545188 & 2.528183 & -0.000021 & $\mathrm{~N}$ & $4.345461-$ & -0.228760 & -0.876256 \\
\hline & 1.264899 & 1.728406 & -0.067032 & & 2.650754 & 2.111437 & -0.690679 \\
\hline & d 0.406004 & -1.097811 & 0.004471 & & 0.438313 & 0.389602 & 0.360370 \\
\hline & -2.893415 & 2.715483 & -0.020143 & & 5.004609 & -1.415384 & -0.899108 \\
\hline & 2.560879 & 0.945109 & -0.024166 & & 1.497722 & 3.068771 & -0.484465 \\
\hline & 2.494763 & -0.405321 & -0.031998 & $\mathrm{~N}$ & 0.329893 & 2.578876 & -0.010609 \\
\hline & 3.800626 & 1.600298 & -0.000878 & & 1.635849 & 4.433897 & -0.779032 \\
\hline C & 3.629112 & -1.125493 & -0.019146 & C & 0.708400 & 3.411848 & 0.174977 \\
\hline & 4.976371 & 0.853631 & 0.009610 & C & 0.560433 & 5.296068 & -0.584503 \\
\hline C & 4.892481 & -0.543210 & 0.000039 & C & 0.643012 & 4.774683 & -0.096220 \\
\hline & -2.196133 & -3.233472 & -0.019940 & $\mathrm{H}$ & 0.410132 & -2.784199 & 1.260101 \\
\hline & -5.457384 & -0.367379 & -0.046335 & $\mathrm{H}$ & 3.297040 & -4.819391 & 0.312020 \\
\hline $\mathrm{H}$ & -4.617345 & -2.716267 & -0.059603 & $\mathrm{H}$ & 0.942581 & -4.841202 & 1.150445 \\
\hline & 1.295267 & 2.524436 & 0.704888 & $\mathrm{H}$ & 2.981174 & 2.172482 & -1.750324 \\
\hline $\mathrm{H}$ & 1.212011 & 2.264610 & -1.041002 & $\mathrm{H}$ & 3.521353 & 2.469116 & -0.098851 \\
\hline & -3.208021 & 3.768468 & -0.051200 & $\mathrm{H}$ & $6.037813-$ & -1.359103 & -1.272117 \\
\hline $\mathrm{H}$ & 3.829312 & 2.693393 & 0.009536 & & 2.591701 & 4.803987 & -1.160415 \\
\hline $\mathrm{H}$ & 3.511740 & -2.214218 & -0.024320 & $\mathrm{H}$ & 1.627864 & 2.957152 & 0.556271 \\
\hline $\mathrm{H}$ & 5.949473 & 1.353659 & 0.027939 & $\mathrm{H}$ & 0.656414 & 6.362380 & -0.810953 \\
\hline $\mathrm{H}$ & 5.786692 & -1.171393 & 0.009123 & & 1.516448 & 5.409817 & 0.071968 \\
\hline & & & & & 3.108793 & -0.863775 & -0.415402 \\
\hline
\end{tabular}




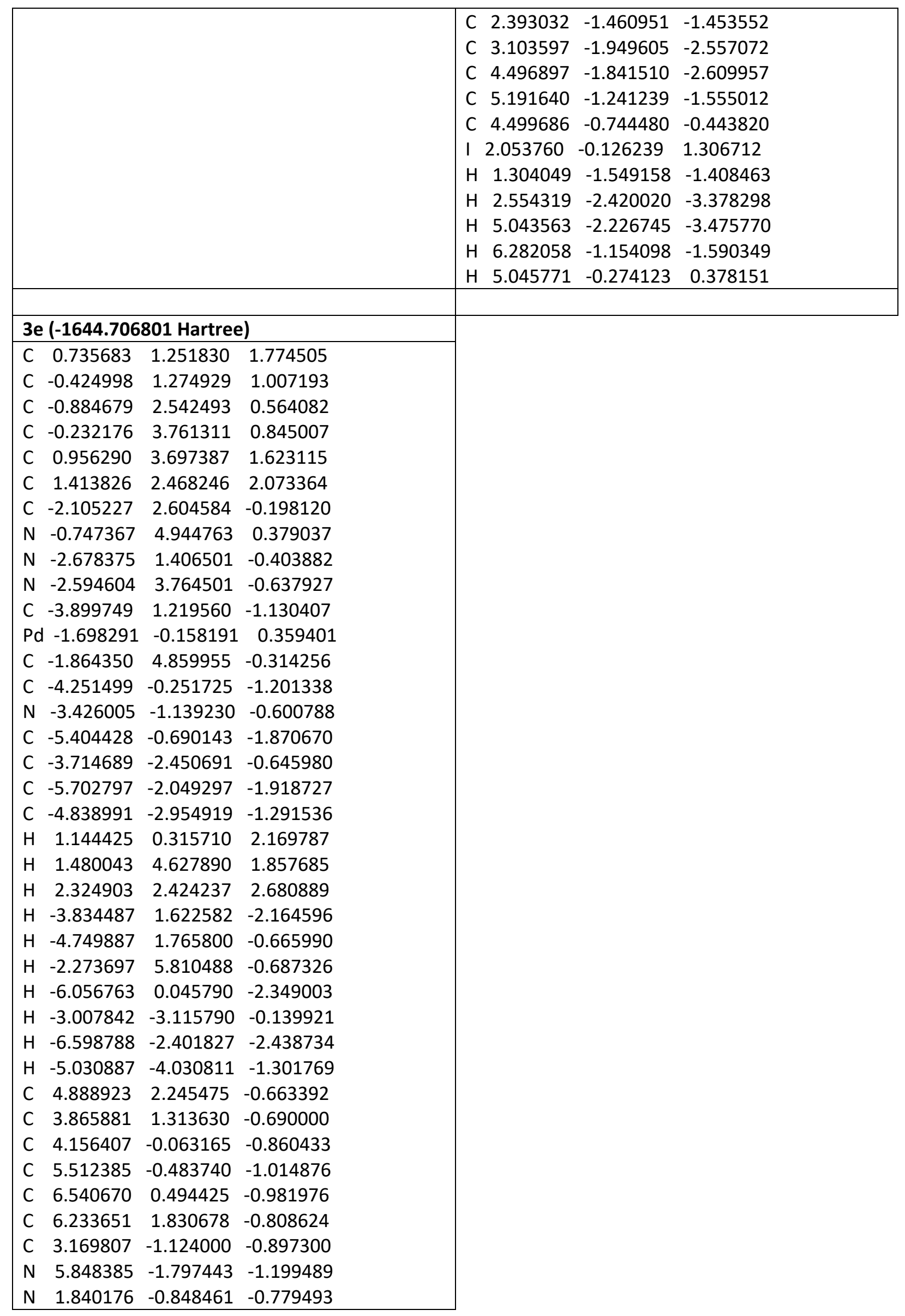




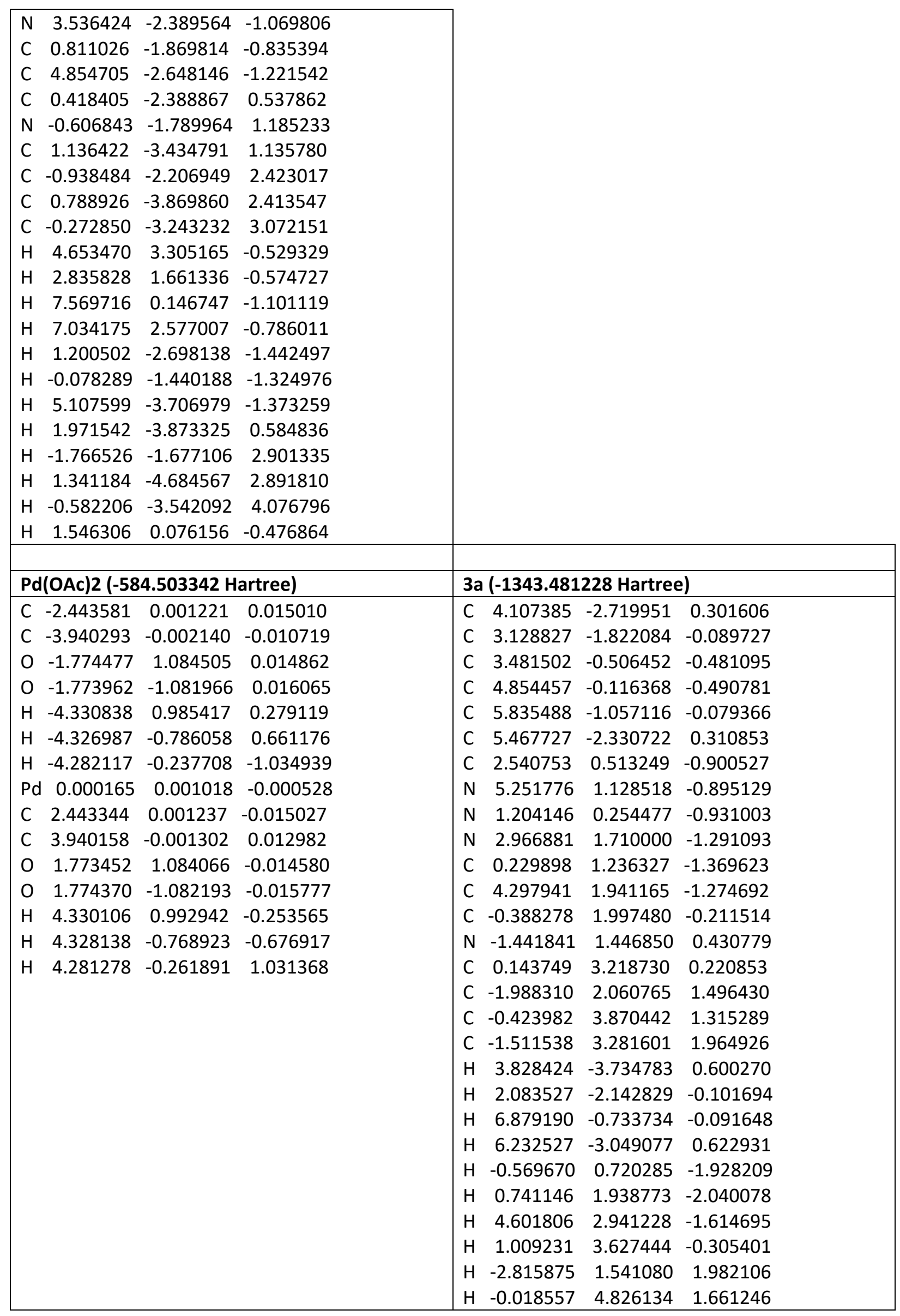




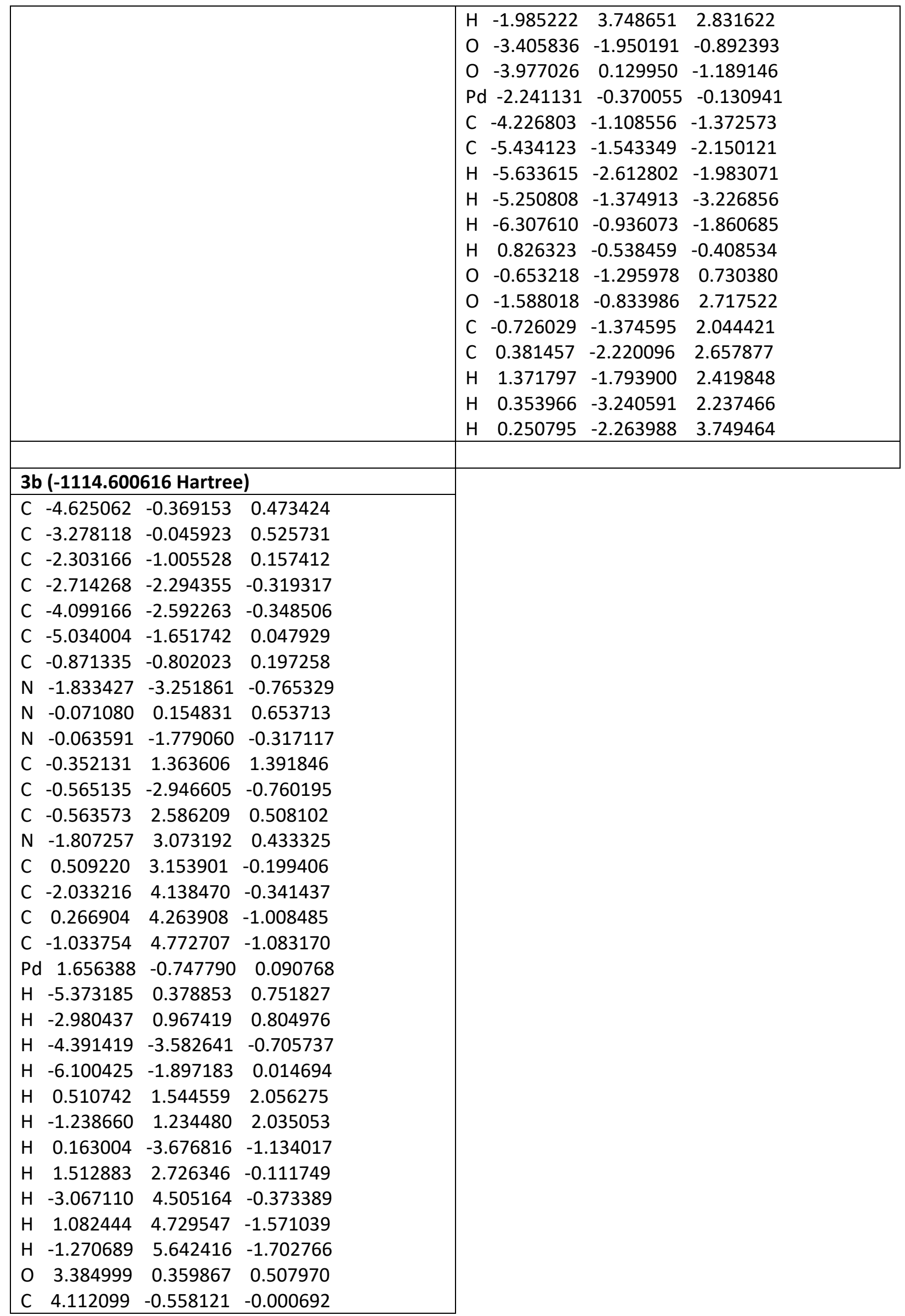




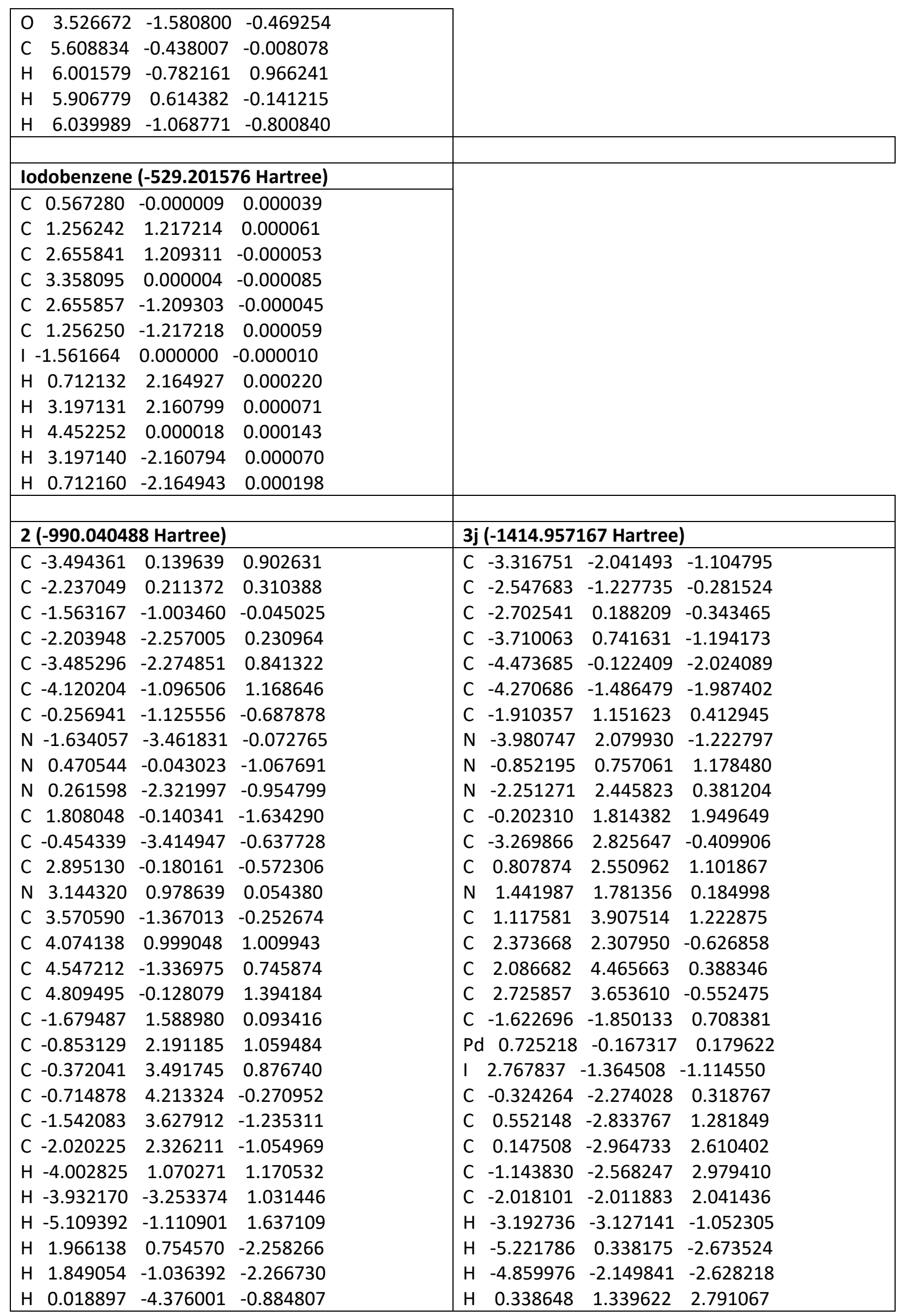




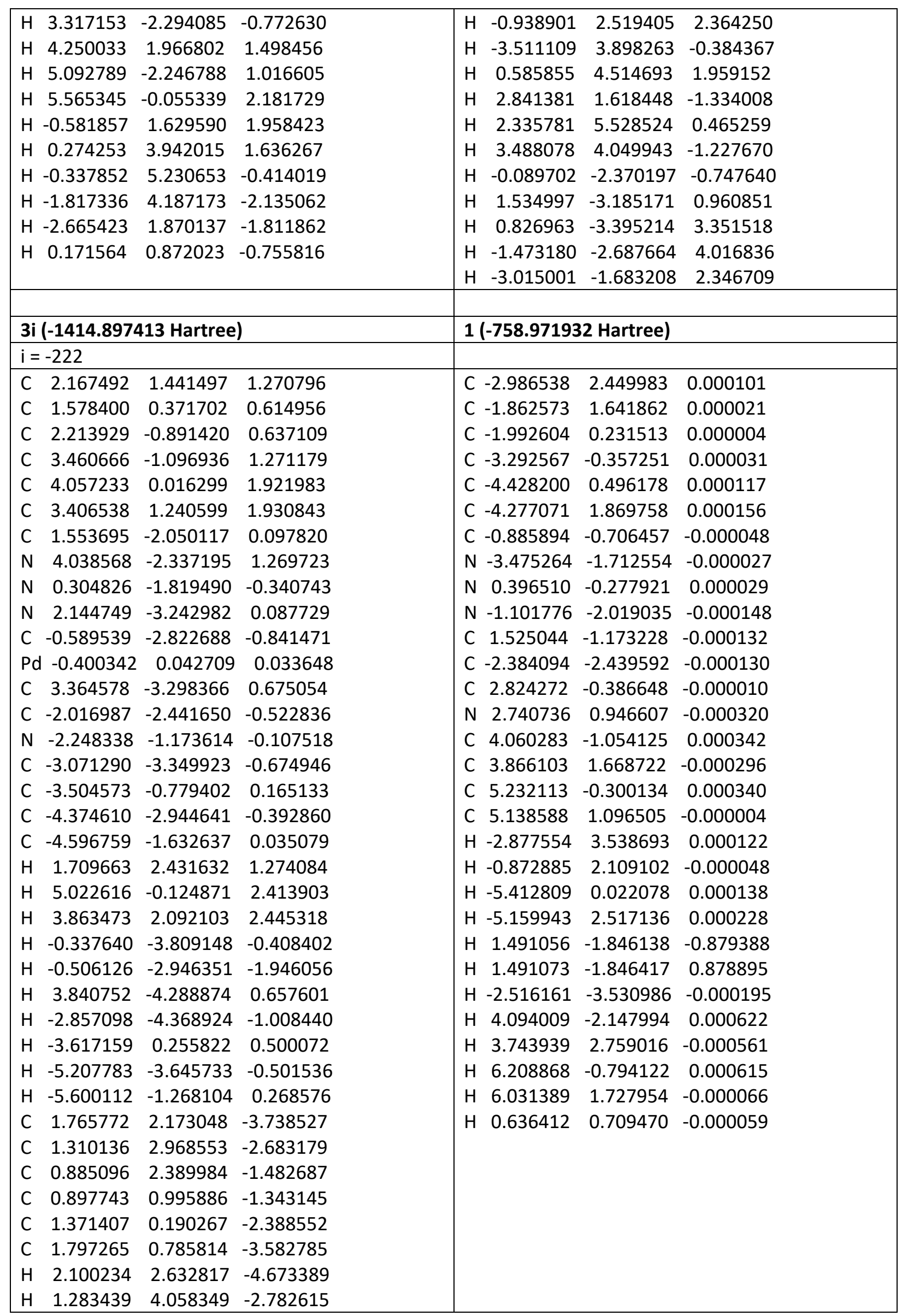




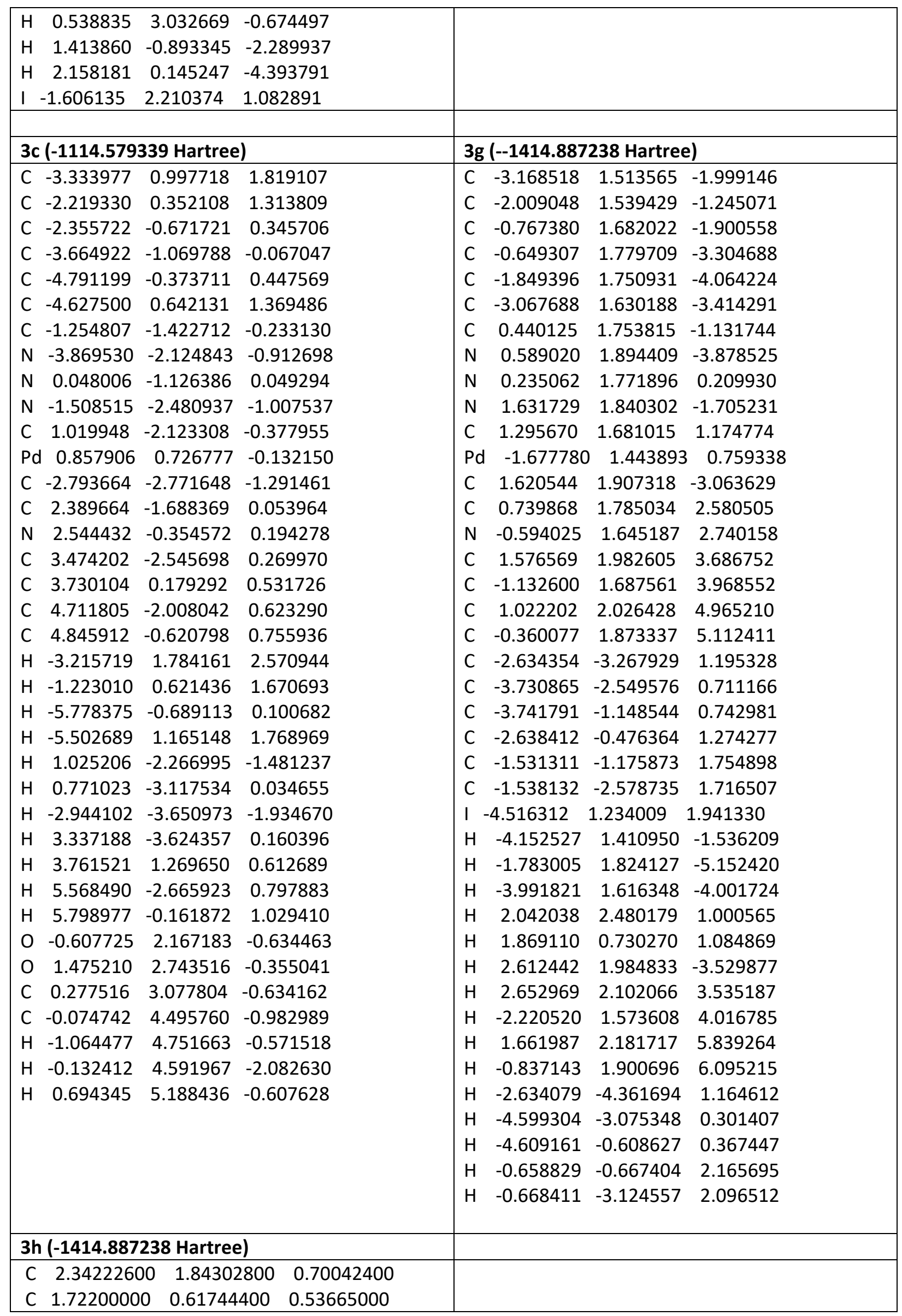




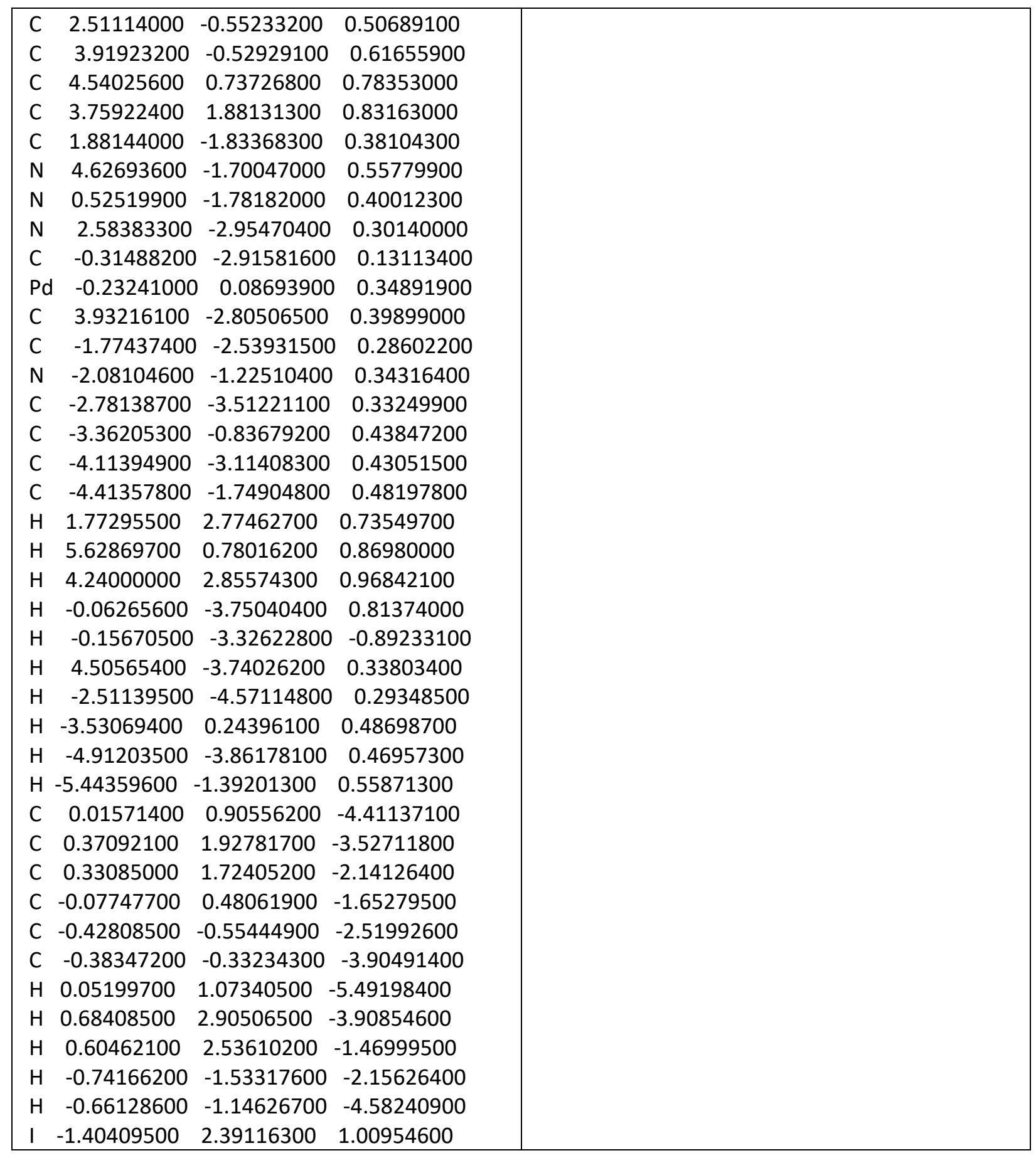


Table S1. Crystal data and structure

refinement Empirical formula

Formula weight

Temperature

Wavelength

Crystal system

Space group

Unit cell dimensions

Volume

Z

Density (calculated)

Absorption coefficient

$\mathrm{F}(000)$

Crystal size

Theta range for data collection

Index ranges

Reflections collected

Independent reflections

Completeness to theta $=26.000^{\circ}$

Absorption correction

Max. and min. transmission

Refinement method

Data / restraints / parameters

Goodness-of-fit on $\mathrm{F}^{2}$

Final $\mathrm{R}$ indices [I $>2 \operatorname{sigma}(\mathrm{I})]$

$\mathrm{R}$ indices (all data)

Extinction coefficient

Largest diff. peak and hole

\section{C32 H35 N7 O3 Pd S}

704.13

$100 \mathrm{~K}$

$0.71073 \AA$

Monoclinic

P 1 21/n 1

$\mathrm{a}=19.2701(14) \AA \quad \alpha=90^{\circ}$.

$\mathrm{b}=8.2426(6) \AA \quad \beta=115.560(2)^{\circ}$.

$\mathrm{c}=20.8740(16) \AA \quad \gamma=90^{\circ}$.

2991.1(4) $\AA^{3}$

4

$1.564 \mathrm{Mg} / \mathrm{m}^{3}$

$0.738 \mathrm{~mm}^{-1}$

1448

$0.29 \times 0.08 \times 0.04 \mathrm{~mm}^{3}$

1.907 to $27.122^{\circ}$.

$-24<=\mathrm{h}<=22,-10<=\mathrm{k}<=7,-26<=\mathrm{l}<=25$

17092

$6309[\mathrm{R}(\mathrm{int})=0.0477]$

$96.0 \%$

Semi-empirical from equivalents

0.0943 and 0.0601

Full-matrix least-squares on $\mathrm{F}^{2}$

6309 / 0 / 400

1.042

$\mathrm{R} 1=0.0489, \mathrm{wR} 2=0.1258$

$\mathrm{R} 1=0.0749, \mathrm{wR} 2=0.1423$

$\mathrm{n} / \mathrm{a}$

1.021 and -0.766 e. $\AA^{-3}$ 


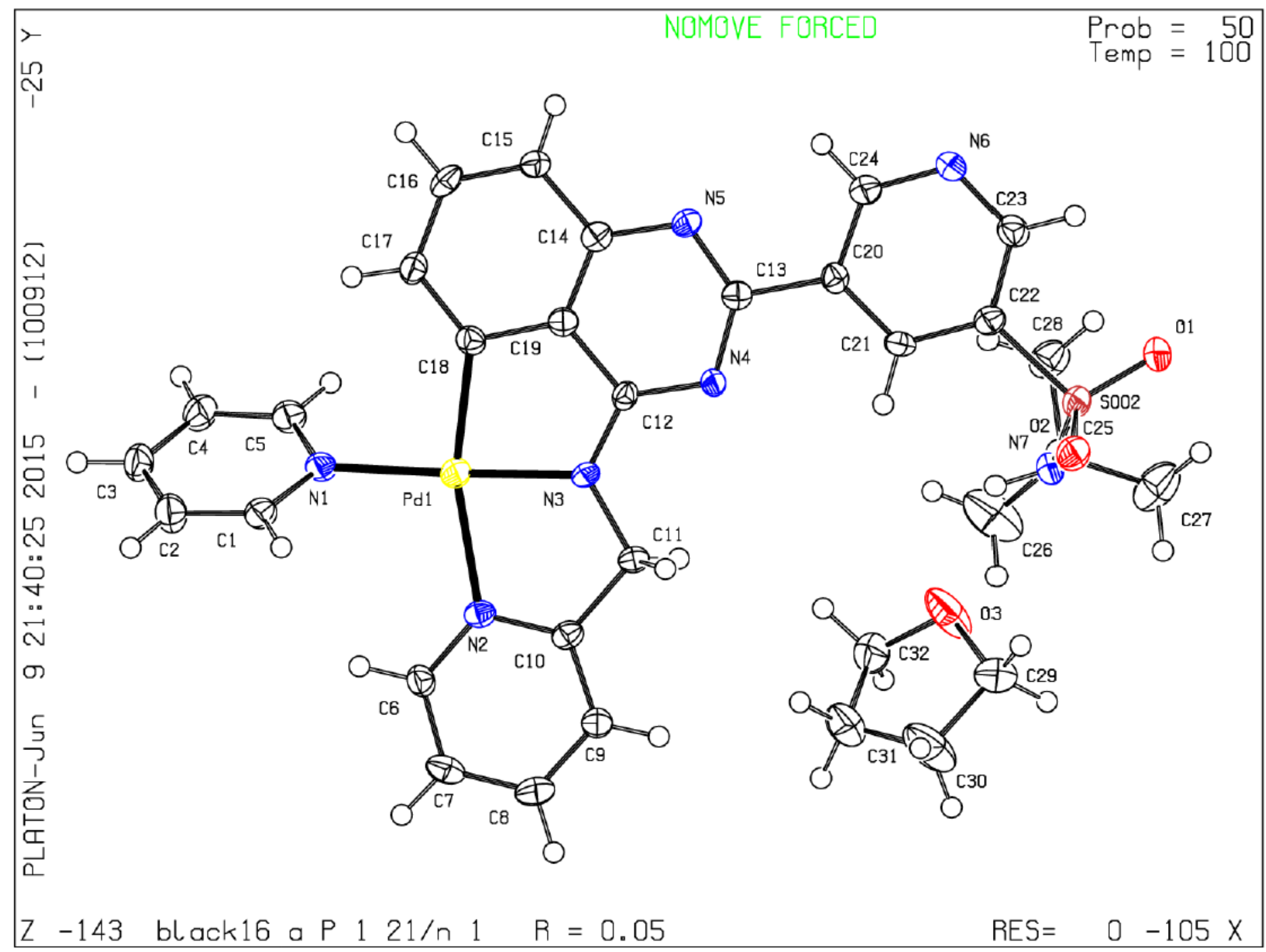

Figure S1. ORTEP diagram for palladacycle 4 shown at 50\% ellipsoid contour probability level.

Procedure for Palladacycle Formation (4). Under ambient conditions, 1 (30 mg, $0.067 \mathrm{mmol}$ ) was dissolved in anhydrous THF. One equivalent of $\mathrm{Pd}(\mathrm{OAc})_{2}(15 \mathrm{mg}, 0.067 \mathrm{mmol})$ was added with stirring. A yellow non-crystalline precipitate formed after $30 \mathrm{~min}$. The solution was cooled to -78 ${ }^{\circ} \mathrm{C}$ and layered with $20 \mathrm{~mL}$ of hexanes. Then, 5 equiv of pyridine $(0.34 \mathrm{mmol})$ was added to the hexanes layer. The reaction tube was placed in a dewar packed with dry ice and allowed to warm to room temperature. After 3 days, golden-yellow, needle-shaped crystals formed at the hexanes/THF interface. The remaining product was isolated to give $17 \mathrm{mg}$ of 4 as the mono-THF solvate in $44 \%$ yield. 
Competency of the Palladcycle in the C-H Activation

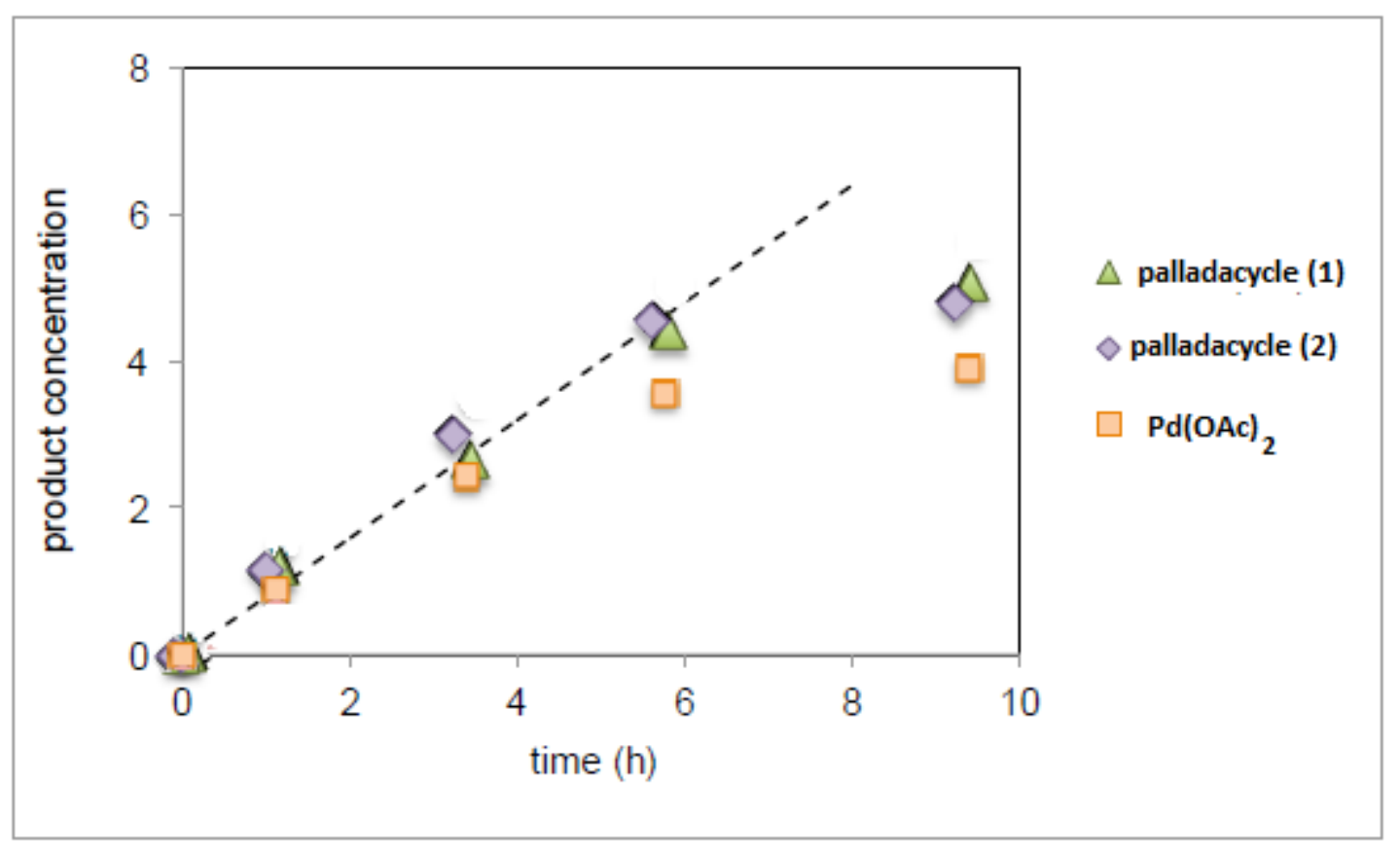


Product Inhibition Study
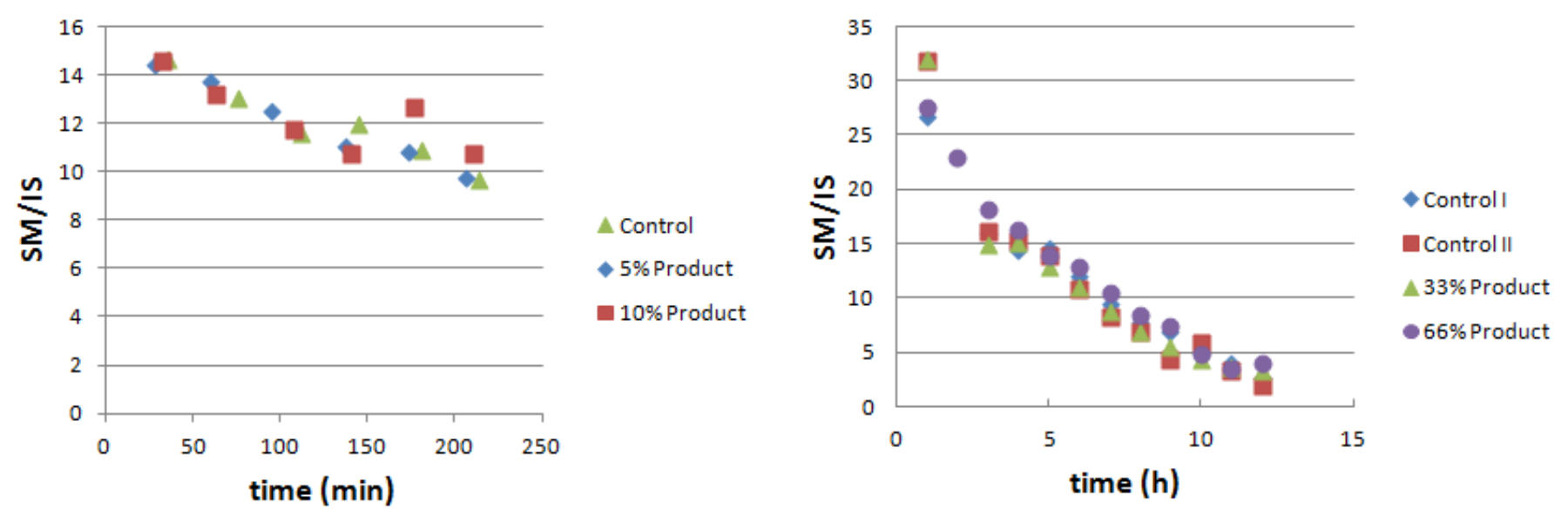

Product Inhibition Study. BMT-212070 (1.0 equiv), Phl (10 equiv), Product (0.0-0.66 equiv), $\mathrm{Pd}(\mathrm{OAc})_{2}(5 \mathrm{~mol} \%)$, $\mathrm{AcOH}$ (0.1 equiv), $\mathrm{KHCO}_{3}$ (3.0 equiv), Anisole $(4 \mathrm{vol}), 113{ }^{\circ} \mathrm{C}$ 
Kinetic Study

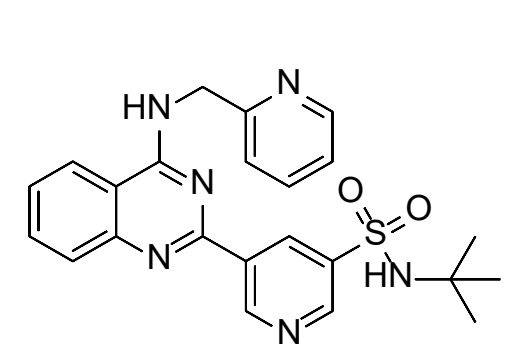

1
$\mathrm{Pd}(\mathrm{OAc}) 2(5 \mathrm{~mol} \%)$

$\mathrm{Phl}$ (12 equiv) KOPiv (3.0 equiv)

Anisole (5 vol) $110^{\circ} \mathrm{C}$

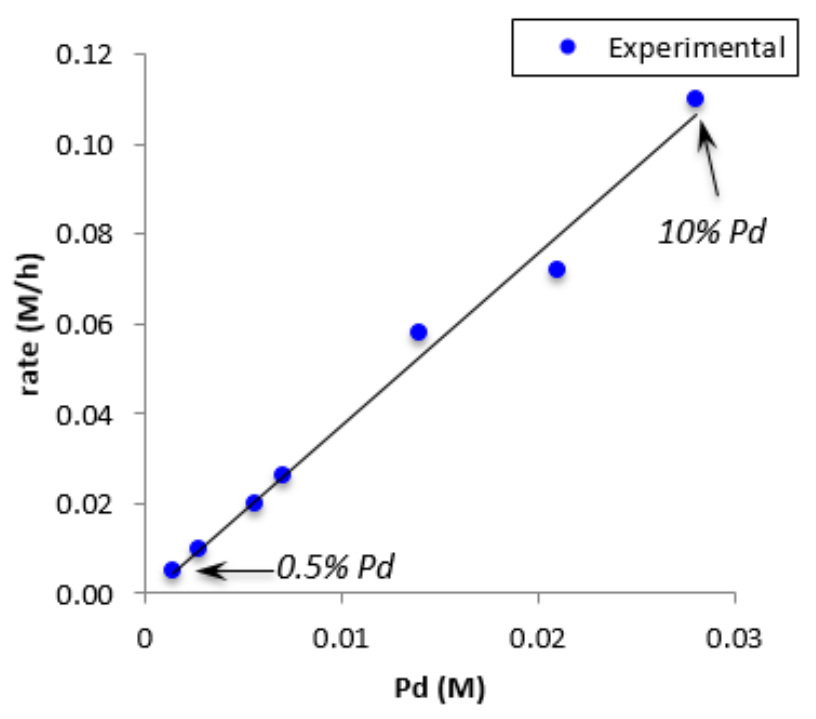

First order in $P_{d}$

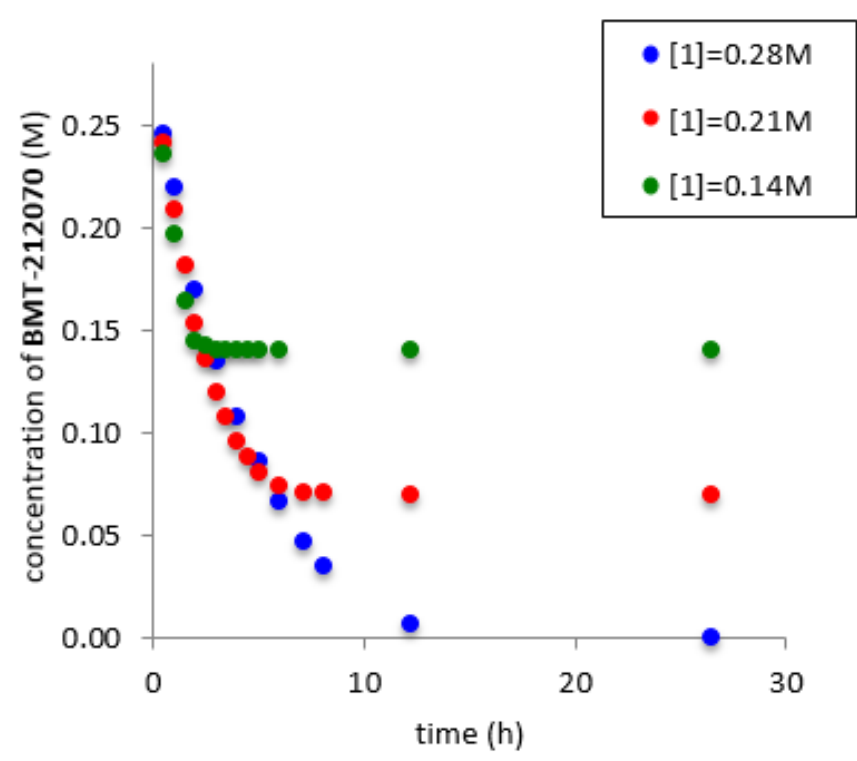

zero order in BMT-212070 


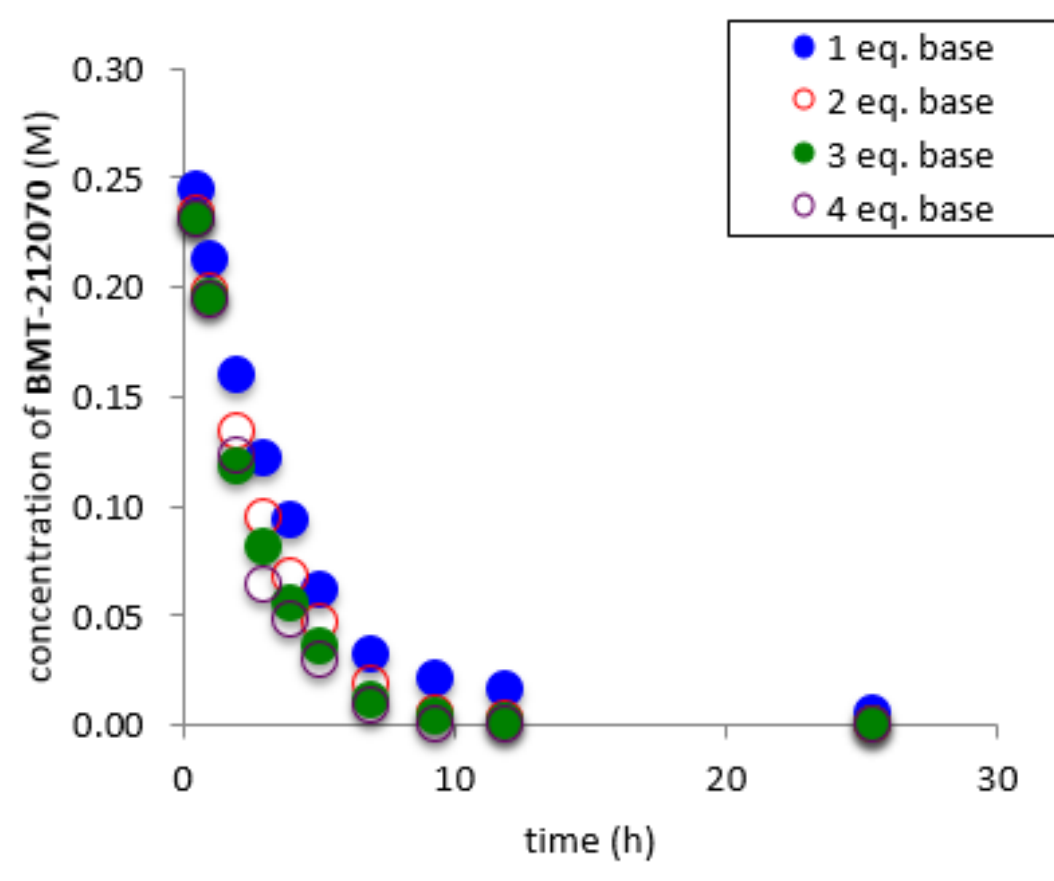

zero order in (KOPiv)

for ca. 50\% conversion

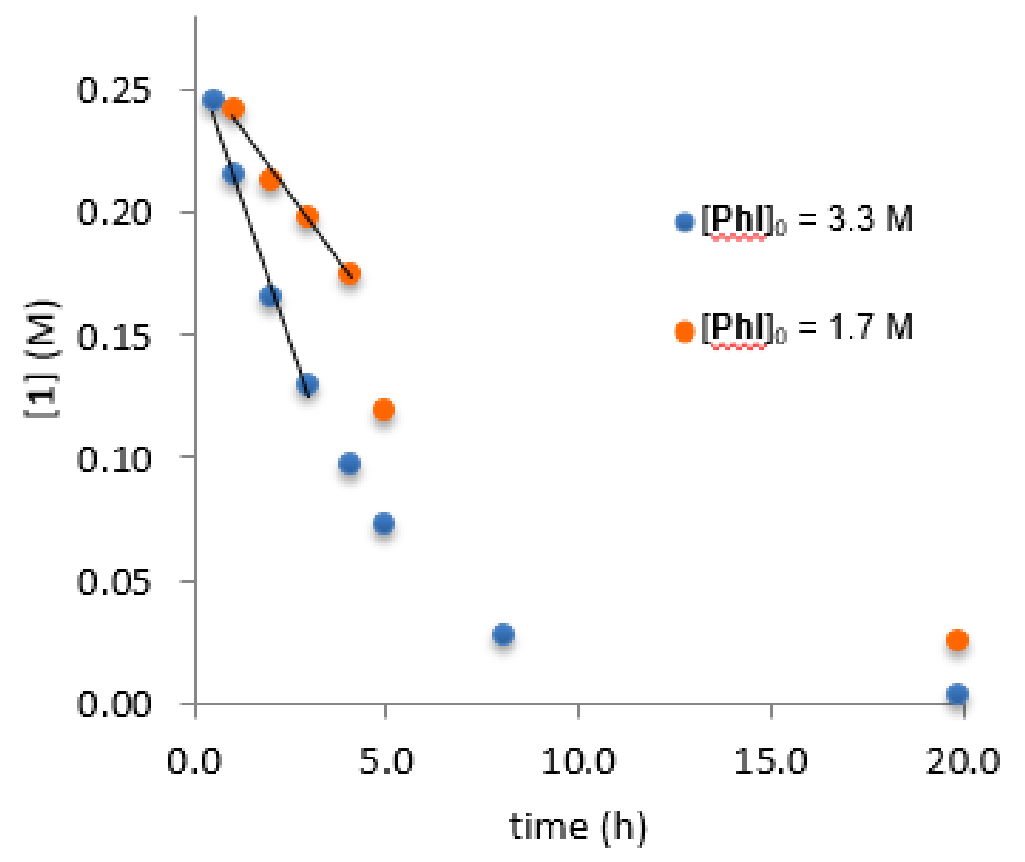

First Order in Phl 
${ }^{1}$ Frisch, M. J.; Trucks, G. W.; Schlegel, H. B.; Scuseria, G. E.; Robb, M. A.; Cheeseman, J. R.; Scalmani, G.; Barone, V.; Mennucci, B.; Petersson, G. A.; Nakatsuji, H.; Caricato, M.; Li, X.; Hratchian, H. P.; Izmaylov, A. F.; Bloino, J.; Zheng, G.; Sonnenberg, J. L.; Hada, M.; Ehara, M.; Toyota, K.; Fukuda, R.; Hasegawa, J.; Ishida, M.; Nakajima, T.; Honda, Y.; Kitao, O.; Nakai, H.; Vreven, T.; Montgomery, J. A. Jr.; Peralta, J. E.; Ogliaro, F.; Bearpark, M.; Heyd, J. J.; Brothers, E.; Kudin, K. N.; Staroverov, V. N.; Kobayashi, R.; Normand, J.; Raghavachari, K.; Rendell, A.; Burant, J. C.; Iyengar, S. S.; Tomasi, J.; Cossi, M.; Rega, N.; Millam, J. M.; Klene, M.; Knox, J. E.; Cross, J. B.; Bakken, V.; Adamo, C.; Jaramillo, J.; Gomperts, R.; Stratmann, R. E.; Yazyev, O.; Austin, A. J.; Cammi, R.; Pomelli, C.; Ochterski, J. W.; Martin, R. L.; Morokuma, K.; Zakrzewski, V. G.; Voth, G. A.; Salvador, P.; Dannenberg, J. J.; Dapprich, S.; Daniels, A. D.; Farkas, Ö.; Foresman, J. B.; Ortiz, J. V.; Cioslowski, J.; Fox, D. J. Gaussian 09, Revision D.01; Gaussian Inc: Wallingford, 2009.

${ }^{2}$ Becke, A.D. Density-functional thermochemistry. III. The role of exact exchange. J. Chem. Phys. 1993, 98, 5648-5652.

${ }^{3}$ Weigend, F.; Ahlrichs, R. Balanced basis sets of split valence, triple zeta valence and quadruple zeta valence quality for $\mathrm{H}$ to Rn: Design and assessment of accuracy. Phys. Chem. Chem. Phys. 2005, 7, 3297-3305. 
${ }^{1} \mathrm{H}$ NMR (500 MHz, DMF-d 7 ) of N-(tert-buty1)-5-(7-(pyridin-2-ylmethy1)-7H-pyrimido[4,5,6-gh]phenanthridin-5-y1)pyridine-3-sulfonamide (3a)

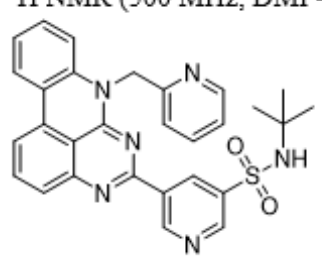

$\underset{\infty}{\infty}$

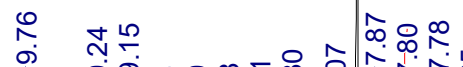

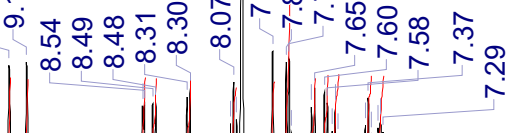

$\stackrel{\frac{2}{6}}{\circ}$

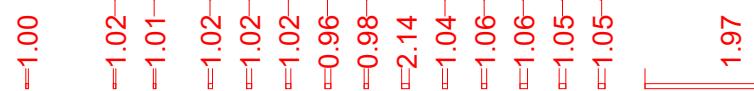



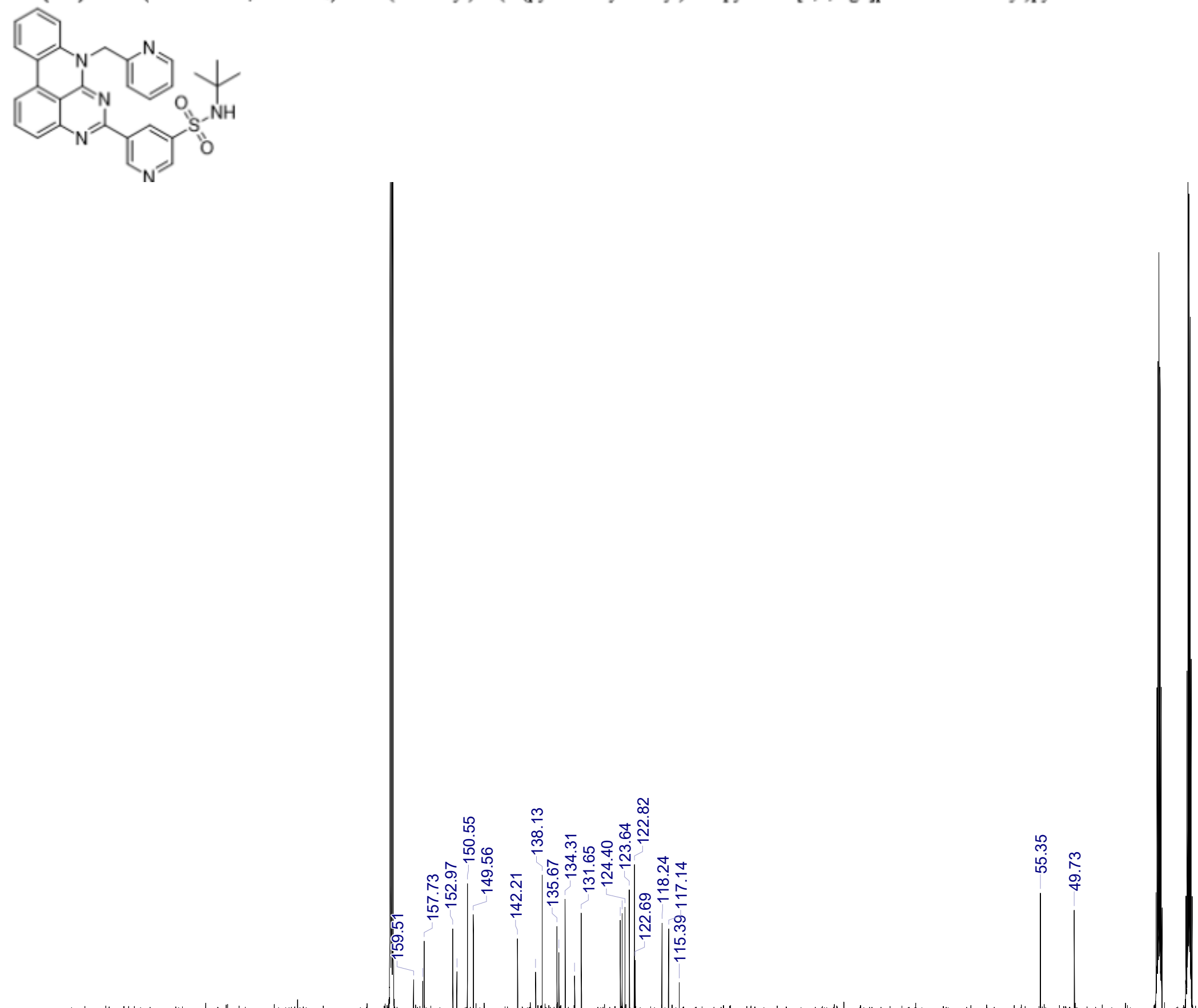

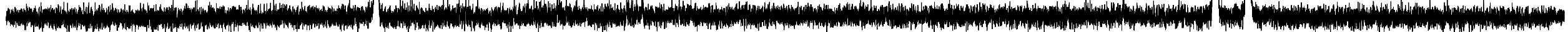



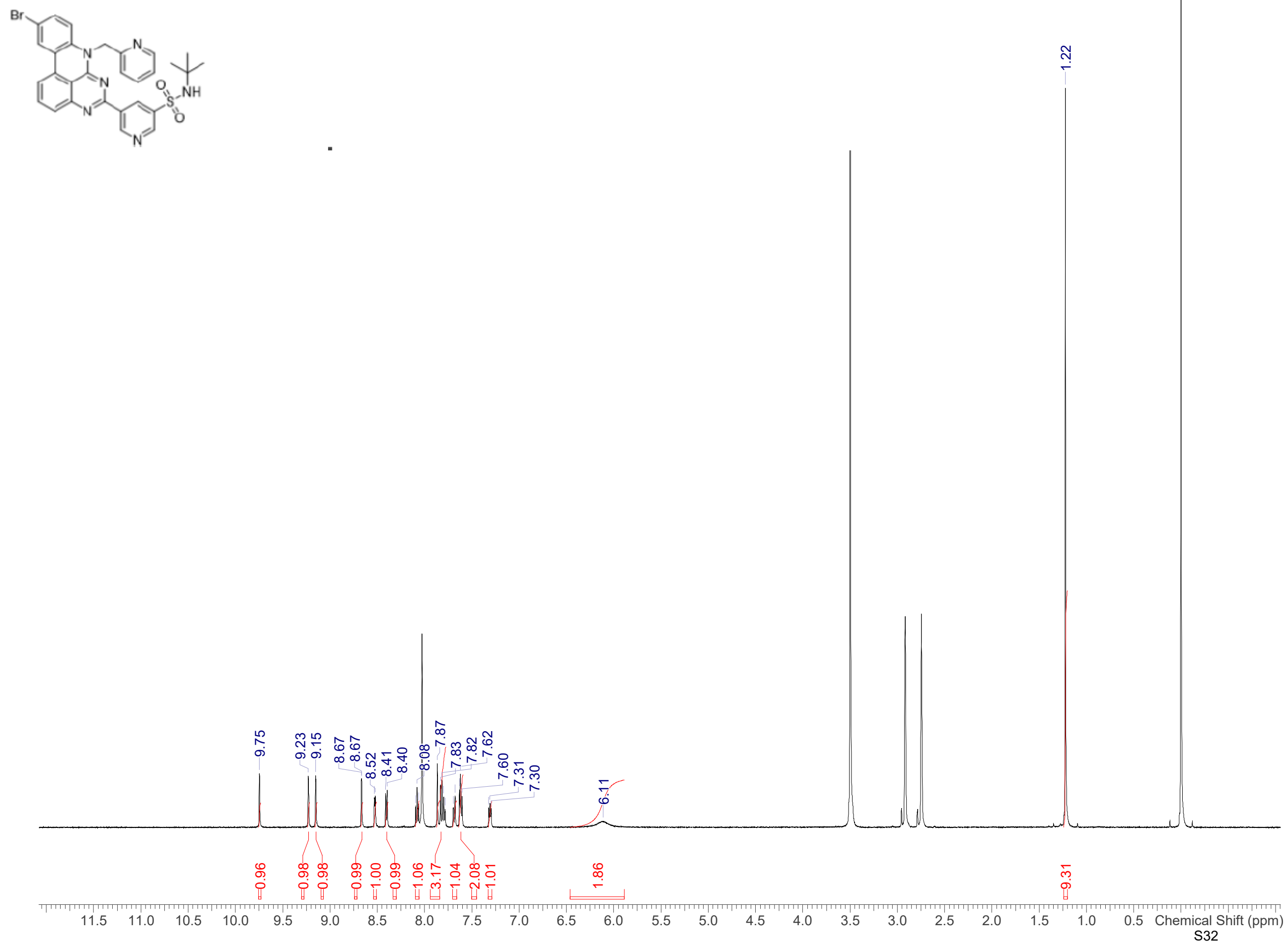


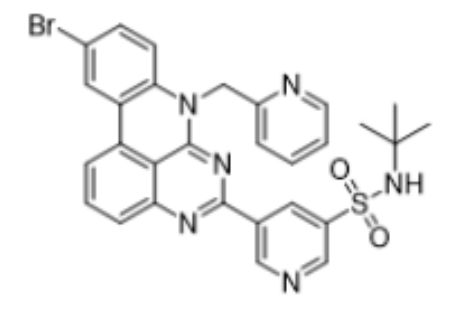

 
${ }^{13} \mathrm{C}\{1 \mathrm{H}\} \mathrm{NMR}(125.8 \mathrm{MHz}, \mathrm{DMF}-\mathrm{d} 7)$ of N-(tert-butyl)-5-(9-fluoro-7-(pyridin-2-ylmethyl)-7H-pyrimido[4,5,6-gh]phenanthridin-5-y1)pyridine-3-sulfonamide (3c)

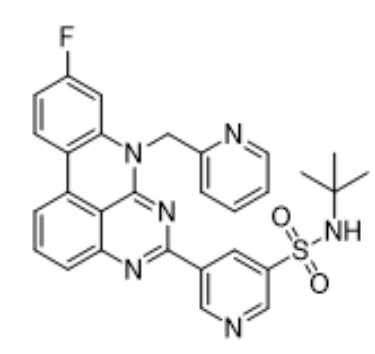

$\stackrel{\infty}{\stackrel{\infty}{\sim}}$

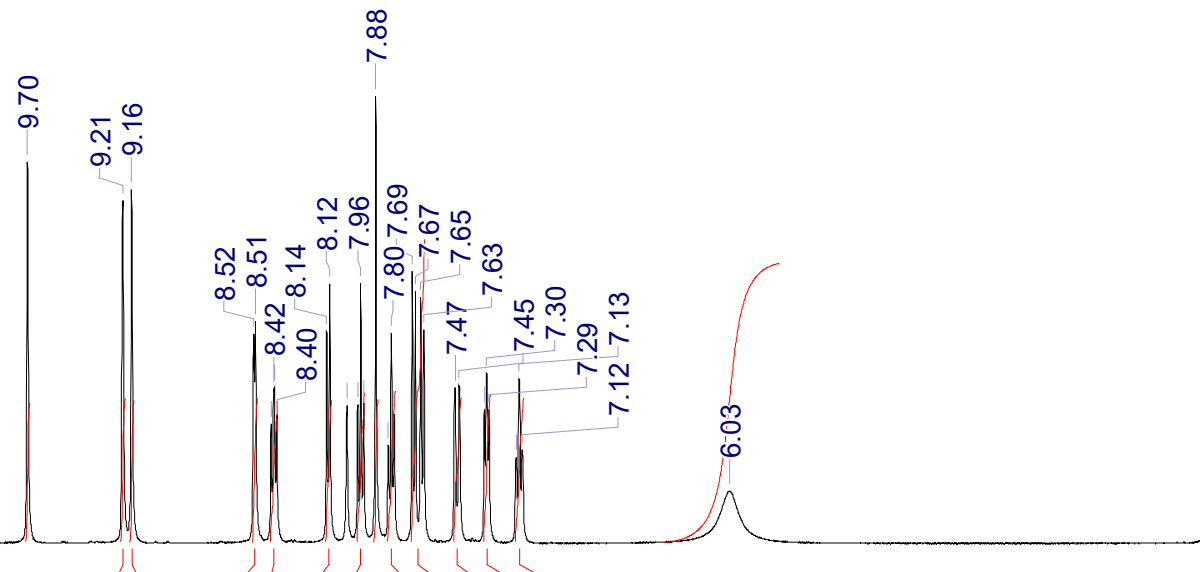

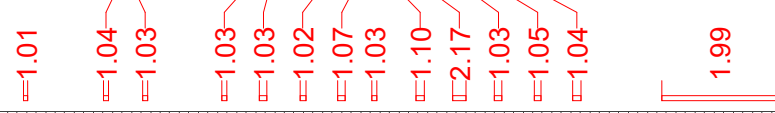



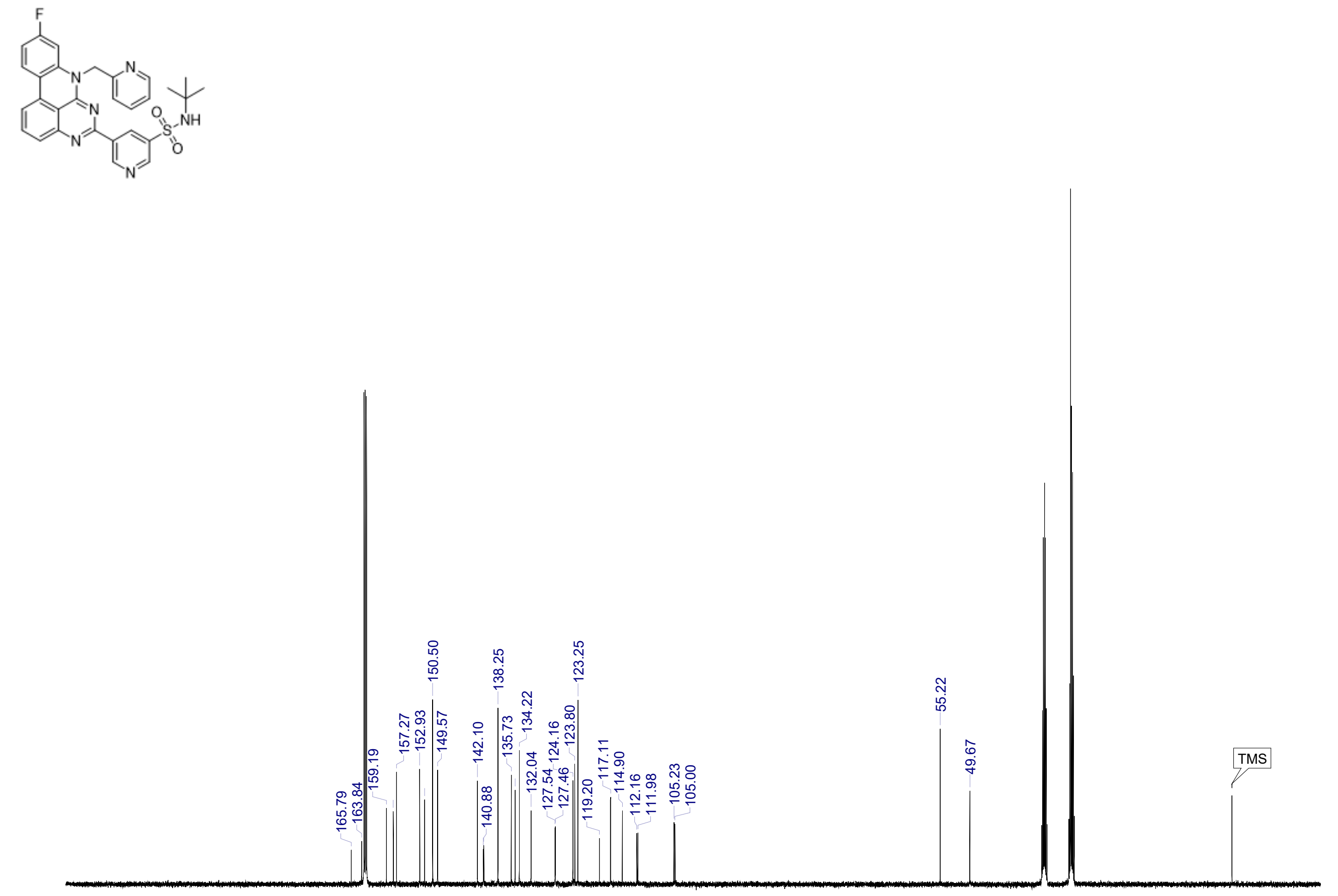


\section{${ }^{13} \mathrm{C}\{1 \mathrm{H}\} \mathrm{NMR}$ (125.8 MHz, DMF-d 7 ) of N-(tert-butyl)-5-(9-methoxy-7-(pyridin-2-ylmethyl)-7H-pyrimido[4,5,6-gh]phenanthridin-5-yl)pyridine-3-}

sulfonamide (3d)

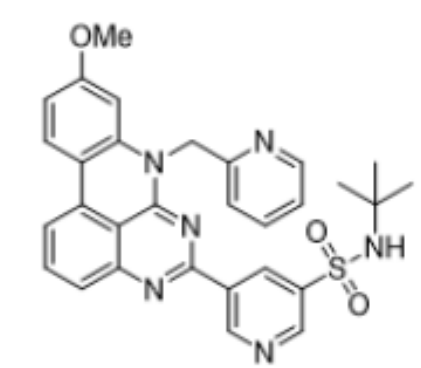

ก.

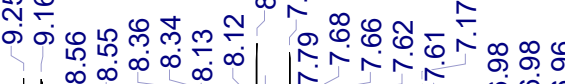

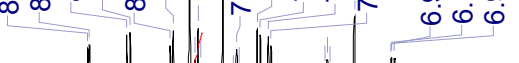
$\underset{6}{\check{0}}$

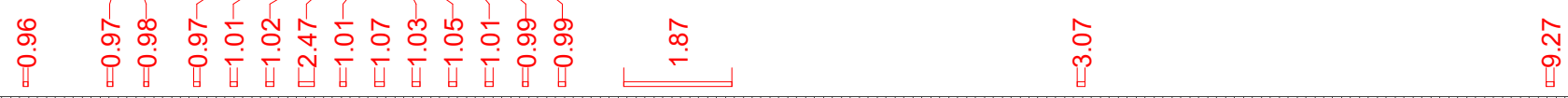




\section{${ }^{13} \mathrm{C}\{1 \mathrm{H}\} \mathrm{NMR}$ (125.8 MHz, DMF-d7) of N-(tert-buty1)-5-(9-methoxy-7-(pyridin-2-ylmethyl)-7H-pyrimido[4,5,6-gh]phenanthridin-5-y1)pyridine-3-}

sulfonamide (3d)
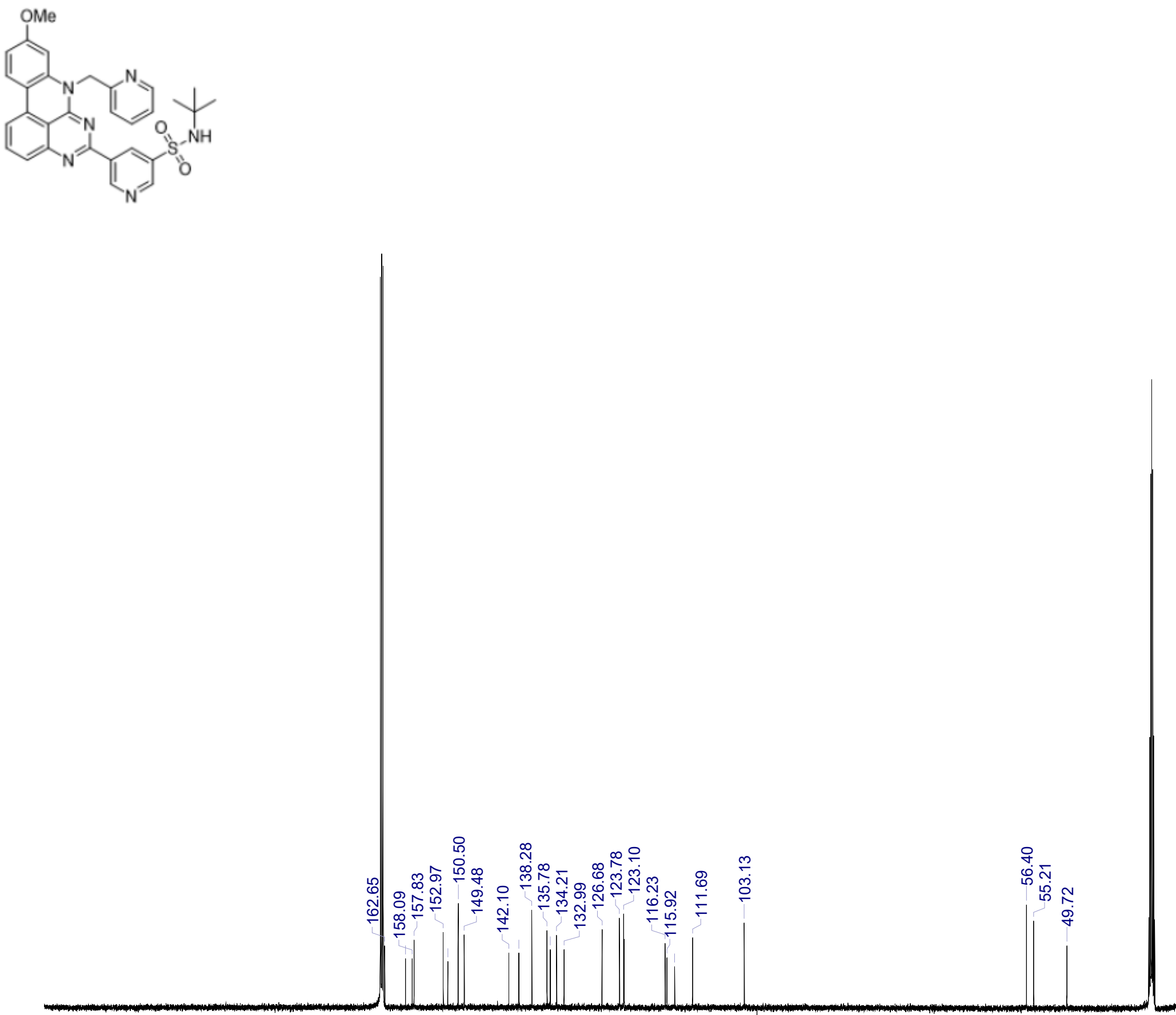
${ }^{13} \mathrm{C}\{1 \mathrm{H}\} \mathrm{NMR}(125.8 \mathrm{MHz}, \mathrm{DMF}-\mathrm{d}$ 7) of N-(tert-butyl)-5-(9-methyl-7-(pyridin-2-ylmethyl)-7H-pyrimido[4,5,6-gh]phenanthridin-5-y1)pyridine-3-sulfonamide (3e)

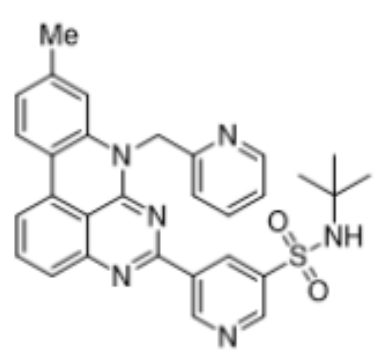

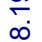

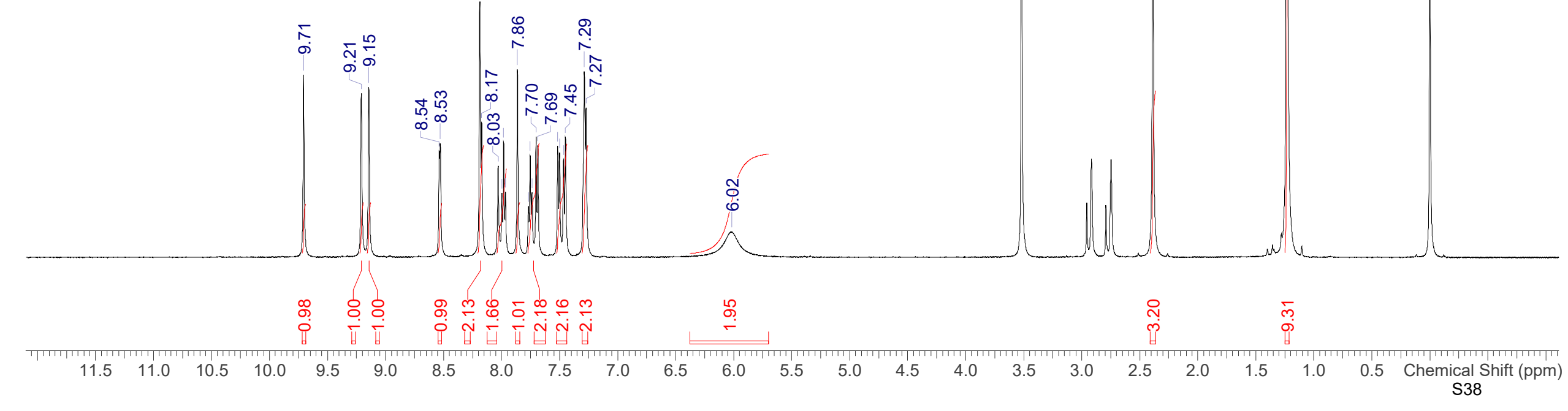



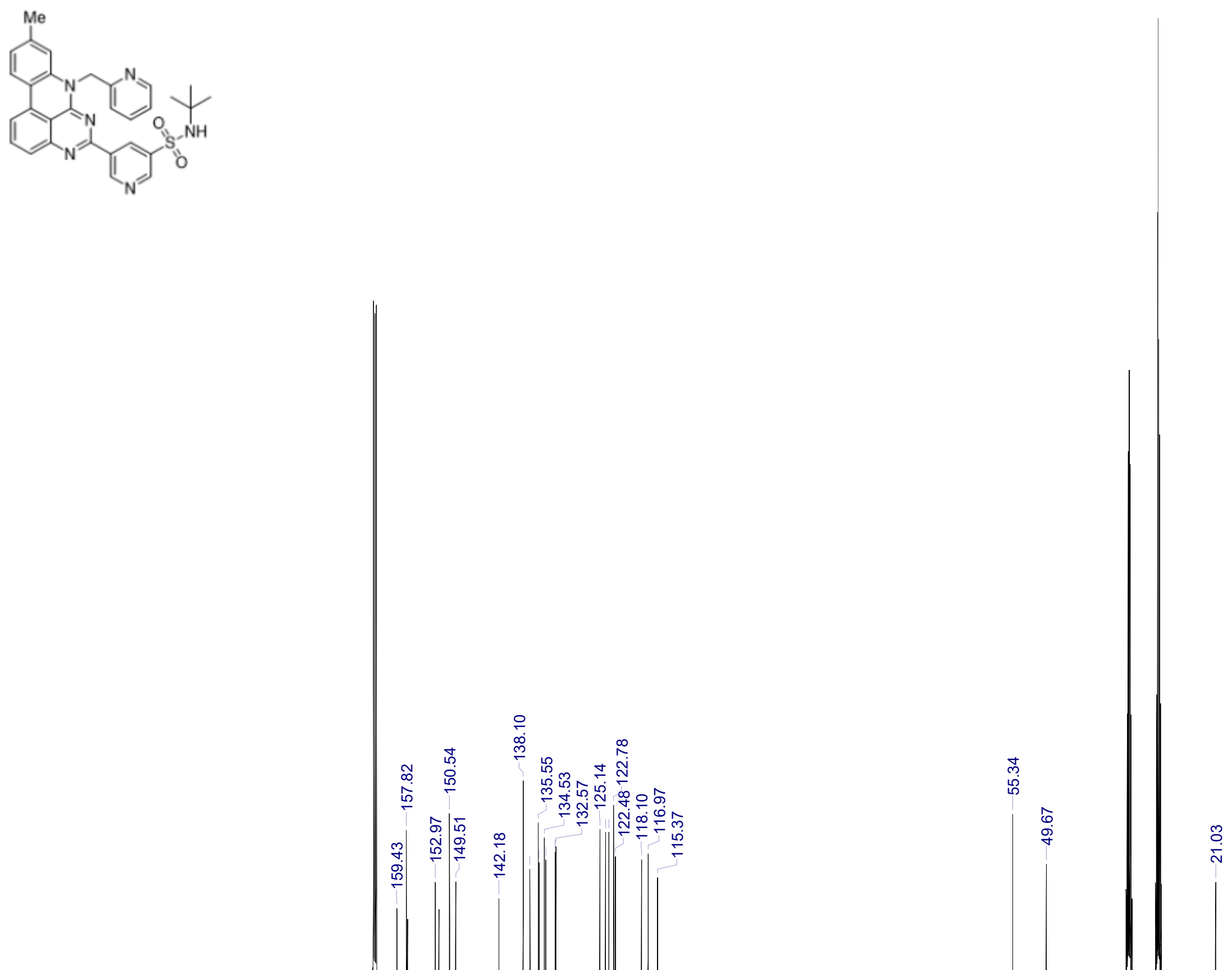

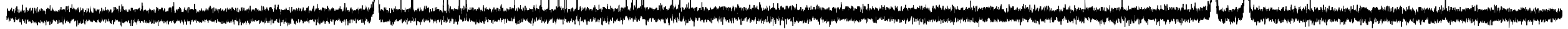


${ }^{13} \mathrm{C}\{1 \mathrm{H}\} \mathrm{NMR}$ (125.8 MHz, DMF-d 7 ) of N-(tert-butyl)-5-(10-methyl-7-(pyridin-2-ylmethyl)-7H-pyrimido[4,5,6-gh]phenanthridin-5-y1)pyridine-3-sulfonamic (3f)

M
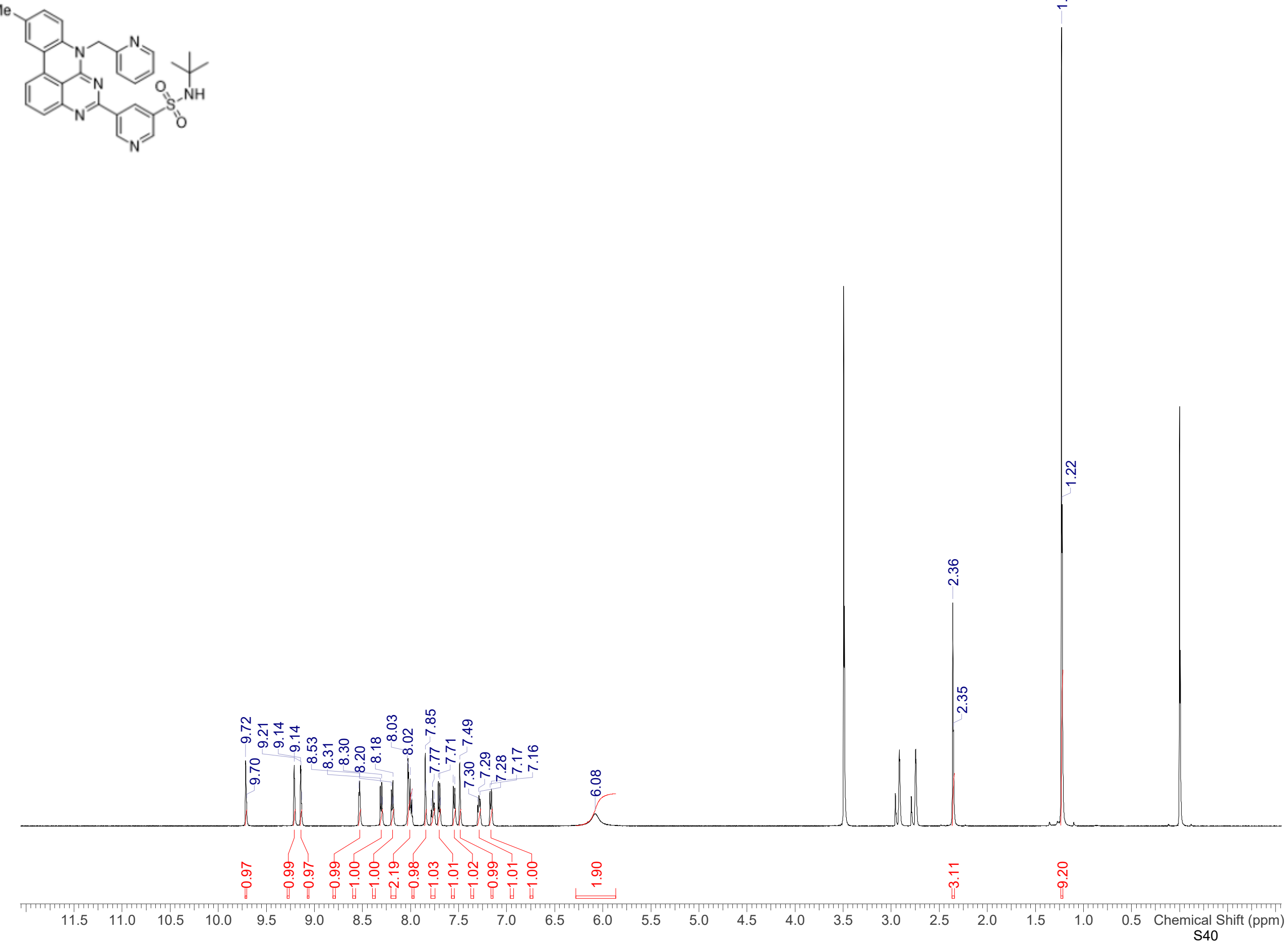
${ }^{13} \mathrm{C}\{1 \mathrm{H}\} \mathrm{NMR}(125.8 \mathrm{MHz}, \mathrm{DMF}-\mathrm{d} 7)$ of N-(tert-butyl)-5-(10-methyl-7-(pyridin-2-ylmethyl)-7H-pyrimido[4,5,6-gh]phenanthridin-5-y1)pyridine-3-sulfonamic (3f)
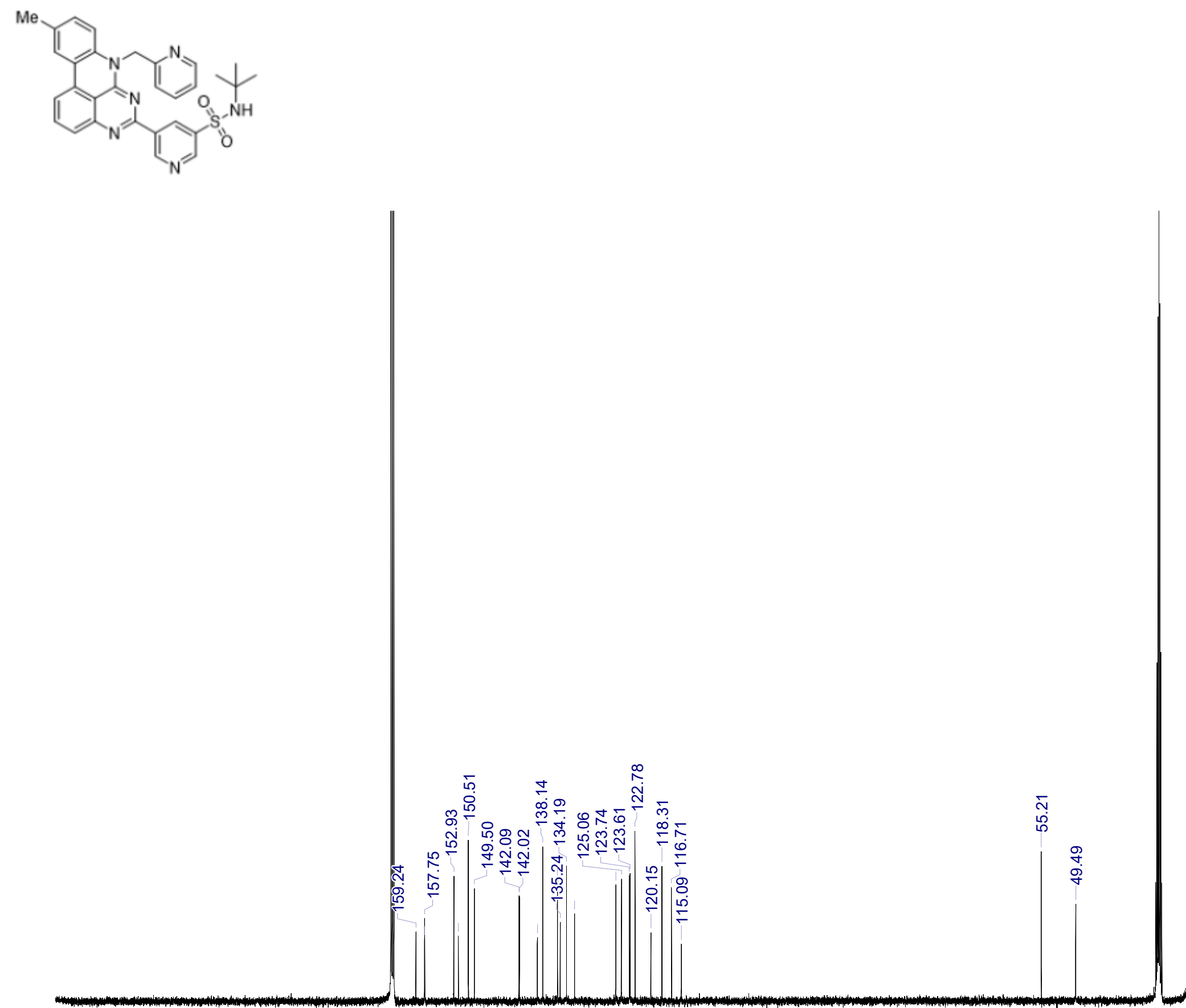